\title{
Low-Energy Threshold Analysis Using the DEAP-3600 Dark Matter Detector
}

\author{
By \\ Jesse Lock \\ A thesis submitted to \\ the Faculty of Graduate and Postdoctoral Affairs \\ in partial fulfillment of the requirements \\ for the degree of
}

Masters of Science

in

Particle Physics

Ottawa-Carleton Institute for Physics

\author{
Department of Physics \\ Carleton University \\ Ottawa, Ontario, Canada
}

September 15, 2019

Copyright (C)

2019 - Jesse Lock 


\section{Abstract}

DEAP-3600 is a single-phase liquid argon WIMP detector located $2 \mathrm{~km}$ underground in the SNOLAB research facility. DEAP-3600 is searching for dark matter; an elusive form of matter that was first postulated in the early $20^{\text {th }}$ century, but has not yet been found. The goal of the following work is to perform a low mass WIMP search. A low mass WIMP search is made possible by lowering the energy threshold of the hardware trigger from 1000 ADC to 150 ADC. Low energy events in the detector are characterized as Cherenkov radiation, low energy retriggers, or high energy retriggers; all of which are background events. The final data set has a live time of 2.10 days and an exposure of $4.41 \times 10^{3} \mathrm{~kg} \cdot$ days using a LAr target mass of $(3279 \pm 97) \mathrm{kg}$. The results from the low mass WIMP search is a WIMP-nucleon spin

independent cross-section of $3.96 \times 10^{-42} \mathrm{~cm}^{2}$ at a $90 \%$ CL corresponding to WIMPs with a mass of $55 \mathrm{GeV} / \mathrm{c}^{2}$. 


\section{Acknowledgements}

I would like to thank my supervisor Dr. Mark Boulay for providing me with the opportunity to work with an excellent research group. I would also like to thank Mark for the support and guidance that he has provided over the course of my Masters Degree. I feel like this has provided me an opportunity to gain unique experiences and learning opportunities that I would not find anywhere else.

I also owe a lot of thanks to Dr. Robert Stainforth. First off, thank you for helping with getting the Cherenkov Monte Carlo working. Thank you for the continual support that you have provided over the course of this work and analysis. You were always willing to meet and answer all of my ridiculous questions, no matter how much you had going on with your own work or how long it took me to understand (sometime much longer then it should have); for this I am very grateful.

I would like to thank the DEAP collaboration as a whole for welcoming me in when I started, it was a great group of people to work with. Thank you to Pierre Gorel and Stefanie Langrock for helping with the modification of the DAQ settings and data collection. I would like to thank all of the collaborators at Carleton for providing guidance and feedback at all of the weekly group meetings. Thanks to Andrew and Carl for answering all of my questions about DEAP and RAT when I first started and throughout my time with DEAP.

I would also like to acknowledge all of the friends that I have made outside of my

research. Dylan Pizzi and Alex Bachiu, you made going through the courses more 
enjoyable. Dylan, you were always there to discuss research, bounce ideas off of, or lets face it, grab a beer and talk about things completely unrelated to our work. My experience at Carleton would not have been the same without you. To all my Jitsu friends, I didn't know what I was getting into when I started; but now I don't know what I would do without it. You always helped me to get my mind off of things when school was getting the best of me, whether it be having laughs or slamming each other into the mats.

Lastly, the people I owe the most thanks to; I would like to thank my family, Dad, Sarah, and Chris for all of their support and love over the course of my entire academic career, it would not have been possible without you guys. I would also like to thank Deb, Scarlett, Santana, and Barry for their support as well.

To Caidence, I'll never understand how you managed to juggle a masters, full time job, part time job, and life; and also dealt with me and everything I was going through. Even with all of this happening you always provided me with constant love and support; I can never express how much this means to me. You've stayed with me through four years apart for undergrad, and now through this; now that it's over I can't wait to start the next chapter of our lives and see what comes next! 


\section{Contents}

Abstract

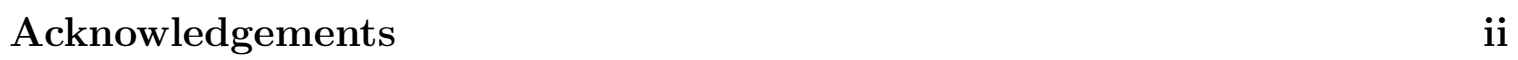

Contents $\quad$ iv

List of Tables vii

List of Figures viii

Nomenclature xi

$\begin{array}{lll}1 & \text { Introduction } & 1\end{array}$

1.1 Overview. . . . . . . . . . . . . . . . . . . . . . . 1

1.2 Evidence for Dark Matter . . . . . . . . . . . . . . . . . 2

1.2.1 Anomalies in Galactic Rotation . . . . . . . . . . . . . 2

1.2.2 The Bullet Cluster . . . . . . . . . . . . . . . . 4

1.2 .3 Cosmic Microwave Background . . . . . . . . . . . . . 5

1.3 Dark Matter Candidates . . . . . . . . . . . . . . . . . . . . . . . . . 7

1.3.1 Weakly Interacting Massive Particles . . . . . . . . . . . . . . 8

1.3 .2 Alternatives . . . . . . . . . . . . . . . . . . 9

1.4 Current Results of Dark Matter Experiments . . . . . . . . . . . . . . 10

1.4 .1 DEAP-3600 . . . . . . . . . . . . . . . . . 12

1.4 .2 Low Mass WIMP Search by DarkSide-50 . . . . . . . . . . . . 13 
\begin{tabular}{lll}
\hline 2 & DEAP-3600 & 15
\end{tabular}

$2.0 .1 \quad$ SNOLAB Underground Research Facility . . . . . . . . . . . . 15

2.1 The Detector . . . . . . . . . . . . . . . . . . . . . 17

2.2 Scintillation in Liquid Argon . . . . . . . . . . . . . . . . . . . . . . 21

2.3 Hardware Trigger System. . . . . . . . . . . . . . . . . . . . . . . . . 22

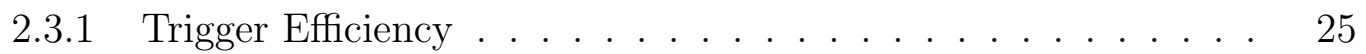

2.4 Pulse-Shape Discrimination . . . . . . . . . . . . . . 26

2.5 Backgrounds . . . . . . . . . . . . . . . . . . . . . . . . . . . . . 28

$2.5 .1{ }^{39} \mathrm{Ar}$ Events $\ldots \ldots \ldots \ldots$

2.5.2 Cherenkov Radiation . . . . . . . . . . . . . . . . . . . 30

$2.5 .3 \quad$ Alphas . . . . . . . . . . . . . . . . . . . 34

2.5 .4 Muons . . . . . . . . . . . . . . . . . . . . 35

\begin{tabular}{|lll}
3 & Random Trigger and Low Threshold Data & 37
\end{tabular}

3.1 Random Trigger . . . . . . . . . . . . . . . . . . . . . . . . . . . 38

3.2 Low-Energy Threshold Data . . . . . . . . . . . . . . . . . . . . . . . 39

3.2.1 Determining the Low-Energy Threshold Trigger Settings . . . 40

3.2 .2 Low-Energy Threshold Data Set . . . . . . . . . . . . . . . . . 41

4 Low-Energy Threshold Analysis 443

4.1 Analysis Variables . . . . . . . . . . . . . . . . . . . . . . . . . . . . . 43

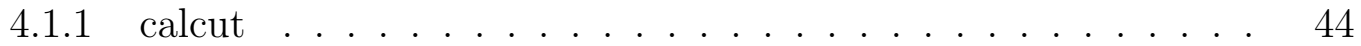

4.1 .2 dtmTrigSrc . . . . . . . . . . . . . . . . . . . . 45

$4.1 .3 \mathrm{qPE} \ldots \ldots \ldots \ldots \ldots$

$4.1 .4 \quad \mathrm{~F}_{\mathrm{prompt}}$ and $\mathrm{F}_{\operatorname{maxPE}} \ldots \ldots \ldots \ldots$. . . . . . . . . . . . 48

$4.1 .5 \quad$ Pile-Up Removal Variables . . . . . . . . . . . . . . . . . . . . 49

4.1 .6 eventTime - Time of the main pulse in a waveform; used for event selections cuts. . . . . . . . . . . . . . . . . . 52 
4.1 .7 Position Reconstruction Variables . . . . . . . . . . . . . . . 53

4.2 Low Threshold Data Processor . . . . . . . . . . . . . . . . . . . . . . 54

4.3 Low Energy Events . . . . . . . . . . . . . . . . . . . . 57

4.3 .1 Retriggers . . . . . . . . . . . . . . . 58

4.3 .2 Cherenkov Events . . . . . . . . . . . . . . . . . . . 63

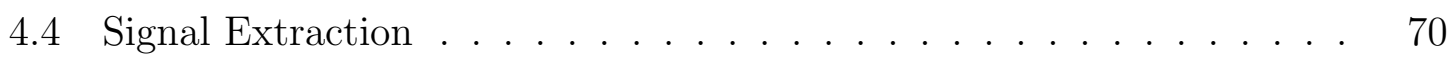

4.4 .1 Nuclear Form Factor . . . . . . . . . . . . . . . . . . . . . . . 71

4.4 .2 Nuclear Recoil Model . . . . . . . . . . . . . . . . . . . . . . . 72

4.4 .3 Total Rate in DEAP-3600 . . . . . . . . . . . . . . . 75

4.4 .4 Exclusion Curve . . . . . . . . . . . . . . . . . . . . . . . . 76

$\begin{array}{lll}5 & \text { Results of Low Mass WIMP Search } & 79\end{array}$

$5.1 \quad$ Final Exposure After Cuts . . . . . . . . . . . . . . . . . . . . . . 79

5.2 Background Subtraction and $\mathrm{S}_{\mathrm{Up}}$. . . . . . . . . . . . . . . . 80

5.3 Low Threshold Analysis Results . . . . . . . . . . . . . . . . . . . . . 82

5.4 Sensitivity Study . . . . . . . . . . . . . . . . . . 85

5.5 Background Study . . . . . . . . . . . . . . . . . 86

$\begin{array}{lll}6 & \text { Conclusions } & 90\end{array}$

6.1 Future Direction . . . . . . . . . . . . . . . . . . . . . . . . 91

\begin{tabular}{lr}
\hline References & 94
\end{tabular}

\begin{tabular}{|l|l|}
\hline Appendix A Determining the Mass of Liquid Argon & 100
\end{tabular} 


\section{List of Tables}

0.1 Commonly Used Abbreviations $\ldots \ldots \ldots \ldots \ldots$ xi

$2.1 \quad$ Gamma Radiation from Norite Rock $\ldots \ldots \ldots$. . . . . . . 17

4.1 List of calcut Bitmasks Used In Event Selection Cuts . . . . . . . . 44

4.2 List of dtmTrigSrc Bitmasks Used In Event Selection Cuts . . . . . . 45

$4.3{ }^{232}$ Th Decay Information . . . . . . . . . . . . . . . . . . . . 64

$4.4{ }^{238} \mathrm{U}$ Decay Information $\ldots \ldots \ldots \ldots$

$5.1 \quad$ Summary of Events that Pass Cuts $\ldots \ldots \ldots \ldots \ldots$ 


\section{List of Figures}

1.1 Galactic Rotation Curve Measurement . . . . . . . . . . . . . . . 3

1.2 Bullet Cluster . . . . . . . . . . . . . . . . . . . . 5

1.3 Cosmic Microwave Background $\ldots \ldots \ldots \ldots$

1.4 Plank 2015 Temperature Power Spectrum . . . . . . . . . . . . 7

$1.5 \quad$ Feynman Diagram of a Dark Matter Particle Scattering off of a Nucle-

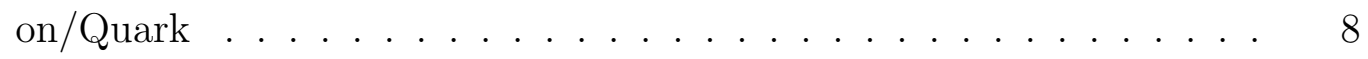

1.6 Dark Matter Candidates . . . . . . . . . . . . . . . . . . . . . . . . . 9

1.7 Exclusion Curves Produced by Various WIMP Search Experiments . 11

1.8 Annual Modulation Results from DAMA . . . . . . . . . . . . . . 12

1.9 Most Recent Results from DEAP-3600 . . . . . . . . . . . . . . 12

1.10 Low Mass WIMP Search Results from DarkSide-50 . . . . . . . . . . 13

$2.1 \quad$ SNOLAB Map $\ldots \ldots \ldots \ldots \ldots \ldots$

2.2 DEAP-3600 Construction Photos $\ldots \ldots \ldots \ldots$

2.3 DEAP-3600 Detector $\ldots \ldots \ldots \ldots \ldots \ldots \ldots$

$2.4 \quad$ DEAP-3600 Data Acquisition System Schematic . . . . . . . . . . 23

2.5 DEAP-3600 Trigger Selection Region Diagram . . . . . . . . . . . 24

2.6 DEAP-3600 Trigger Efficiency $\ldots \ldots \ldots \ldots \ldots \ldots$

$2.7 \quad$ Examples of Electronic and Nuclear Recoils in Data . . . . . . . . 27

$2.8 \quad$ Nuclear and Electronic Recoils in PSD-Space. . . . . . . . . . . . . 28

$2.9{ }^{39}$ Ar Spectrum in DEAP-3600 . . . . . . . . . . . . . . . . 30

$2.10{ }^{40} \mathrm{~K}$ Decay Scheme $\ldots \ldots \ldots \ldots \ldots \ldots \ldots \ldots$ 
$2.11^{232}$ Th Decay Chain . . . . . . . . . . . . . . . . . . . 32

$2.12{ }^{238} \mathrm{U}$ Decay Chain . . . . . . . . . . . . . . . . . . . . . 33

2.13 Surface Alpha Event $\ldots \ldots \ldots \ldots$

3.1 Random Trigger Waveforms $\ldots \ldots \ldots$

3.2 qPE Spectra of Different Trigger Thresholds $\ldots \ldots \ldots \ldots \ldots$

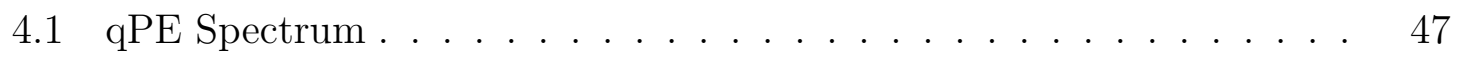

4.2 Low Threshold Trigger qPE Spectrum … . . . . . . . . . . 47

$4.3 \quad$ Waveform With $\mathrm{F}_{\text {prompt }}$ Integration Regions $\ldots \ldots \ldots \ldots$

$4.4 \quad$ Waveform with Multiple Pulses . . . . . . . . . . . . . . 50

4.5 deltaT Distribution of Low Threshold Data. . . . . . . . . . . . 51

$4.6 \quad$ Flow Chart of the Low Threshold Trigger Processor . . . . . . . . . 56

4.7 Comparison Between Low Threshold Data and ${ }^{39} \mathrm{Ar}$ Monte Carlo . . 57

$4.8{ }^{39} \mathrm{Ar}$ Spectrum Used To Create Monte Carlo . . . . . . . . . . . . 59

$4.9 \quad$ Scan of ${ }^{39} \mathrm{Ar}$ Monte Carlo Scan Looking at Position and deltat . . . . 60

4.10 Retriggers in ${ }^{232} \mathrm{Ar}$ Monte Carlo . . . . . . . . . . . . . . . . 61

4.11 Retrigger Removal in Low Threshold Data . . . . . . . . . . . . . 62

4.12 Cherenkov Radiation Produced by ${ }^{232}$ Th Decay in PMT Glass . . . . 64

4.13 Cherenkov Radiation Produced by ${ }^{232}$ Th Decay in LGs . . . . . . . . 65

4.14 Cherenkov Radiation Produced by ${ }^{238} \mathrm{U}$ Decay in PMT Glass . . . . . 65

4.15 Cherenkov Radiation Produced by ${ }^{40} \mathrm{~K}$ Decay in PMT Glass . . . . . 67

4.16 Cherenkov Radiation Produced by ${ }^{40} \mathrm{~K}$ Decay in the LGs . . . . . . 68

$4.17^{39} \mathrm{Ar}$ and Cherenkov Background Subtraction from Data $\ldots . . . .69$

$4.18^{39} \mathrm{Ar}$ and Cherenkov Background Subtraction from Data with all Cuts Applied . . . . . . . . . . . . . . . . . . 70

4.19 Nuclear Form Factor . . . . . . . . . . . . . . . . . . . . . 72

4.20 Nuclear Recoils Spectra in LAr _. . . . . . . . . . . . . . 74 
4.21 Low Threshold Data Trigger Efficiency . . . . . . . . . . . . . . . . . 76

$4.2290 \%$ Upper Limit on Number of Possible WIMPs . . . . . . . . . . . 77

5.1 Background Rate Curve . . . . . . . . . . . . . . . . . . . . . 81

5.2 Efficiency Low Threshold Analysis Compared to Standard DEAP-3600 Analysis . . . . . . . . . . . . . . . . . . . . . 83

5.3 Low Threshold Analysis Exclusion Curve . . . . . . . . . . . . . . . . 84

5.4 Results from Sensitivity Study . . . . . . . . . . . . . . . . . . . . . 86

5.5 Results from Background Study . . . . . . . . . . . . . . . . . . . . . 87

$5.6 \quad$ Results from Background Study if Low Threshold Trigger was Run for 2 Years . . . . . . . . . . . . . . . . . . . 88

6.1 Comparison Between Low Threshold Data and Random Trigger Data 92 


\section{Nomenclature}

A table of abbreviations has been included due to a high number of acronyms used in this thesis. All of these are either commonly used in high energy particle physics or are ways to describe detector components.

\begin{tabular}{|c|c|}
\hline Abbreviation & Definition \\
\hline ADC & Analog-to-Digital Converter \\
\hline AV & Acrylic Vessel \\
\hline $\mathrm{CMB}$ & Cosmic $\underline{\text { Microwave }} \underline{B}$ ackground \\
\hline DAQ & $\underline{\text { Data }} \underline{\underline{\mathbf{A}}}$ cquisition System \\
\hline DEAP & 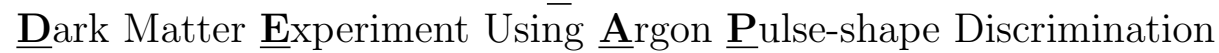 \\
\hline DTM & Digitizer and Trigger Module \\
\hline ER & Electronic Recoil \\
\hline ERB & Electron Recoil Band \\
\hline GAr & Gaseous Argon \\
\hline LAr & Liquid Argon \\
\hline NR & 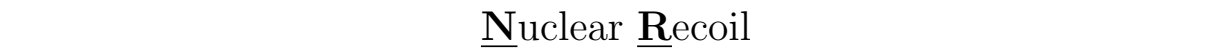 \\
\hline NRB & Nuclear Recoil Band \\
\hline PMT & Photomultiplier Tube \\
\hline PPG & Pulse Pattern Generator \\
\hline PSD & Pulse-shape Discrimination \\
\hline qPE & Total PhotoElectrons \\
\hline RAT & $\underline{\text { Reactor } \underline{A} \text { nalysis Tool }}$ \\
\hline ROI & Region Of Interest \\
\hline $\mathrm{SCB}$ & Signal Conditioning Board \\
\hline TPB & Tetraphenyl Butene \\
\hline VUV & Vacuüm Ultraviolet \\
\hline WIMP & Weakly Interacting Massive Particle \\
\hline
\end{tabular}

Table 0.1: List of abbreviations that are used throughout this thesis. 


\section{Chapter 1}

\section{Introduction}

\section{$1.1 \quad$ Overview}

The focus of the work presented in this thesis is a low energy threshold analysis of events in the DEAP-3600 detector. The goal of this work is to lower the energy threshold of the hardware trigger in the detector in order to increase the sensitivity and perform a low mass WIMP search. Reducing the energy threshold of the trigger allows for the possible detection of not only more WIMP candidates but also lower mass WIMP candidates. In order to complete a low-energy threshold analysis, the main objective is to understand the source of all of the background events in the data within the search region of interest (ROI). For the remainder of this thesis, the term "threshold" will refer to the energy threshold of the hardware trigger system in DEAP-3600 in the context of a dark matter search. To provide motivation behind why a study like this should be conducted, the rest of Chapter 1 will provide a history and description of dark matter as well as current results from various dark matter detection experiments. Chapter 2 will provide a description of the DEAP3600 experiment; why it was built, how it works and the dominant backgrounds that contribute to data. Chapter 3 discusses two ways to obtain low-energy threshold data 
by lowering the trigger energy threshold of the standard physics trigger or by using a specialized hardware trigger called the random trigger. Chapter 4 will provide a description of a low threshold trigger time processor that was created as part of this work as well as a detailed overview of the analysis that was conducted. Chapter 5 shows the results of the low-energy threshold analysis. Lastly, Chapter 6 will discuss the conclusions based on the results that are obtained as well as future work that can improve upon the current work.

\subsection{Evidence for Dark Matter}

In the early 1930's an astronomer named Fritz Zwicky began collecting the first evidence supporting dark matter. Before this point there were suspicions that there was some other constituent to the universe, but with no real evidence. Through the measurement that Zwicky made on the mass of the Coma Cluster, he found that the galaxy contained 400 times more mass then that which could be explained by luminous matter alone $[1-3]$.

Since the measurements done by Zwicky, there has been a large amount of evidence collected that supports the postulation of dark matter. Of this evidence, there are three main pieces; anomalies in the rotation of galaxies, gravitational lensing, and measurements of the cosmic microwave background.

\subsubsection{Anomalies in Galactic Rotation}

In the 1970s, astronomer Vera Rubin was measuring the rotational velocities of spiral galaxies and noticed anomalies when comparing her observations to predictions based on the virial theorem (a classical mechanics theory that relates the kinetic energy, or velocity, of an object to the amount gravitational potential energy acting on the object [1]). Since spiral galaxies are shaped like a disc, the expected behaviour 
of the rotational velocities is that it will increase as the radius approaches the center and decrease as the radius increases (seen as the line labelled "disk" in Figure 1.1). However, the predicted behaviour of spiral galaxies was based on the assumption that all the mass in the galaxy is contained only in luminous matter (an object made of matter that can be seen). When V. Rubin made these measurements [4 6] she found that this is not in fact how spiral galaxies rotate.

DISTRIBUTION OF DARK MATTER IN NGC 3198

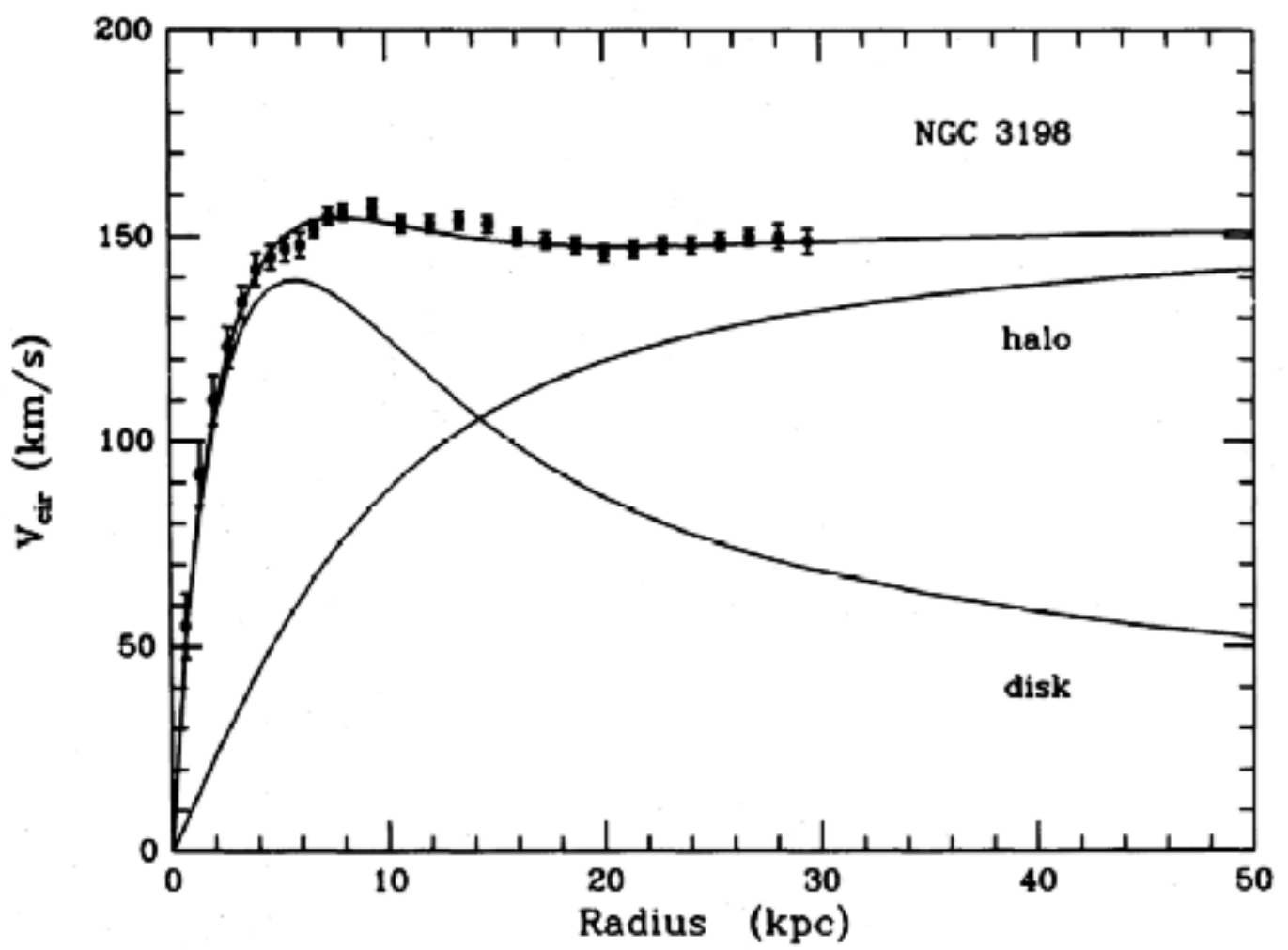

Figure 1.1: NGC 3198 galactic rotational velocity curve 7]. The expected galactic rotational velocity is labelled with "disk" and the measured galactic rotational velocity is labelled with "NGC 3198".

Measurements (NGC 3198 curve in Figure 1.1) indicate that the rotational velocity of galaxies increases sharply near the center of the galaxy then remains relatively constant through the remainder of the galaxy to the edges. Anomalies in rotational velocity as a function of radial distance to the center of the galaxy indicates that 
the galaxy must contain a much larger mass in addition to what can be measured as luminous matter. To account for these differences, it is postulated that there is a large quantity of non-luminous mass throughout galaxies known as the dark matter halo. This can be seen Figure 1.1 as the line labelled "halo". It can be seen that the addition of the halo compensates for the lack of rotational velocity of a disc at high radii, while the disc dominates the velocity contribution at low radii.

\subsubsection{The Bullet Cluster}

Further evidence for dark matter can be seen in gravitational lensing. Gravitational lensing is a phenomena in which a large mass can cause the distortion and bending of electromagnetic radiation (light). Gravitational lensing is commonly seen around galaxies and black holes and can provide a method for measuring the mass of the object in question, since the amount that the light is bent $(\theta)$ due to gravitational lensing is proportional to the mass of the object (given by Equation 1.1] 8].

$$
\theta=\frac{4 G M}{r c^{2}}
$$

where $\mathrm{G}$ is the gravitational constant, $\mathrm{M}$ is the mass of the object, $\mathrm{r}$ is the radial distance between the mass and the light ray, and c is the speed of light.

The bullet cluster is an example of a case where two galaxies have passed through one another which allows for an investigation of the gravitational effects of one galaxy on another. Figure 1.2 shows images of the bullet cluster taken by the Magellan IMACs Telescope (left) and an x-ray image taken by the Chandra X-ray Observatory (right). The green contours on these images shows the center of mass as measured by gravitational lensing. From the x-ray image it can be seen that the majority of the gas and plasma (baryonic matter) from the galaxies is contained around the central point of the collision. This does not agree with the the central point of mass of each of the 
two galaxies as defined by gravitational lensing. To explain these findings, researchers have interpreted that there is a very large mass of a weakly interacting (or possibly non-interacting due to the fact that the contents of each galaxy passed through one another, seemingly with no interaction) non-luminous type of matter (dark matter) that is causing this magnitude of gravitational lensing [9]. The amount that the light bends indicates that there is $\sim 7: 1$ ratio of dark matter + galaxy constituents to the amount of plasma [9].
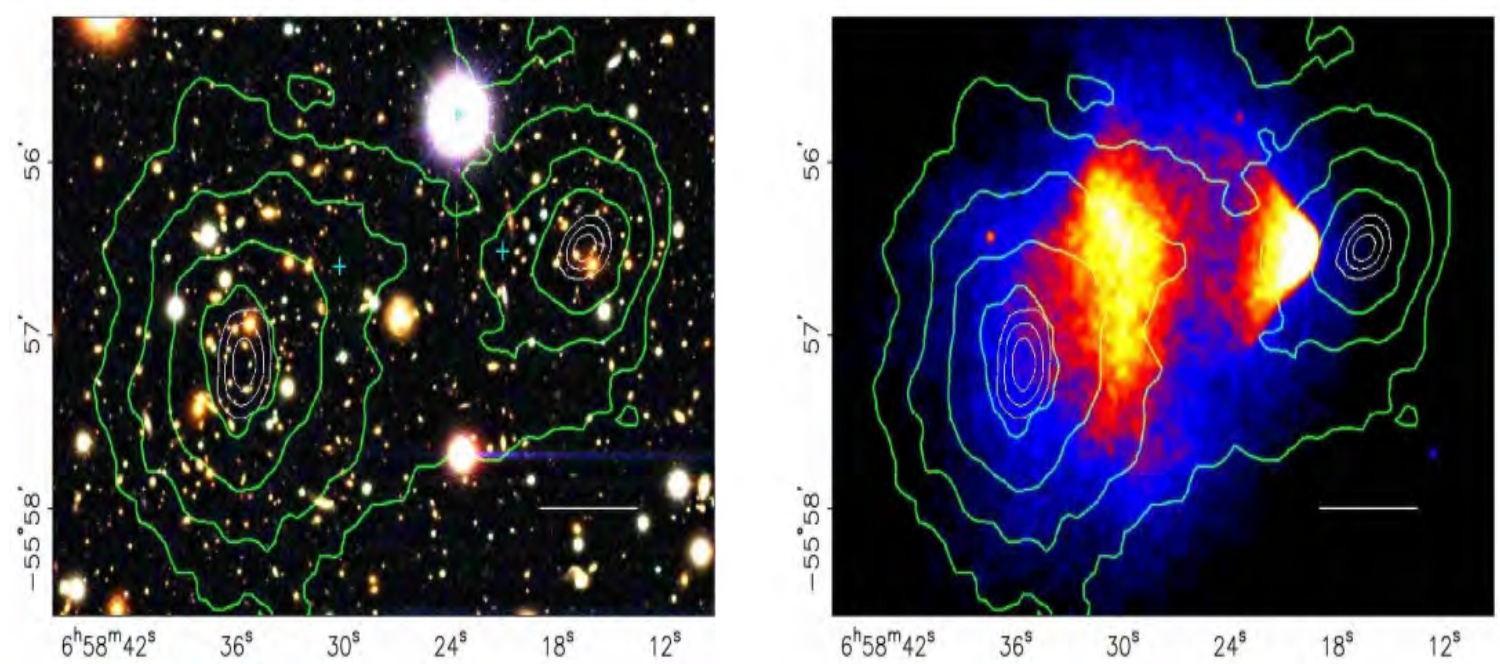

Figure 1.2: Analysis of the gravitational centers of the bullet cluster [9]. The photo on the left was taken by the Magellan IMACs Telescope and the photo on the right is an X-ray image taken by the Chandra X-ray Observatory. The green contours show the gravitational centers calculated by gravitational lensing.

\subsubsection{Cosmic Microwave Background}

The early universe started as a very dense mass of matter that accelerated in all directions very rapidly, this event is known as the big bang. The Cosmic Microwave Background (CMB) is a measure of all of the light in the universe a very short amount of time after matter recombination. Due to properties of general relativity and the expansion of the universe, this light has been redshifted to the microwave frequency range. The CMB is nearly isotropic in temperature; Figure 1.3 shows a heat map of 
the CMB with small temperature changes on the scale of $\mu \mathrm{K}$.

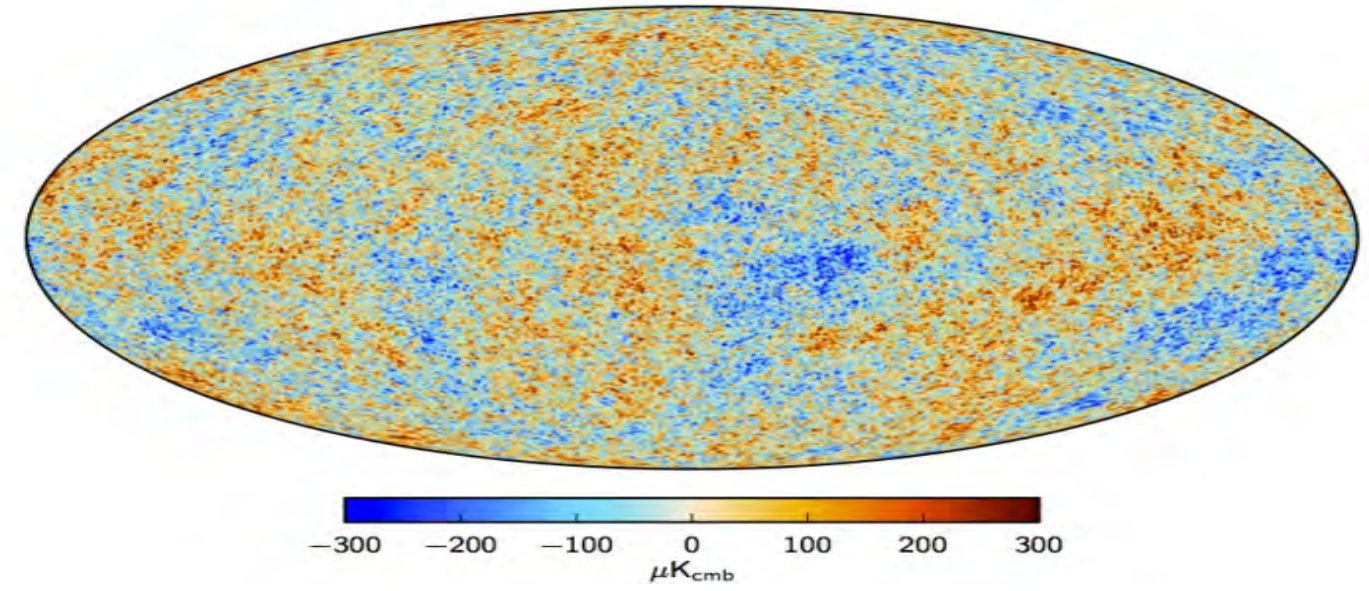

Figure 1.3: Cosmic Microwave Background [10]. Map of the early universe where the warmer clusters correspond to regions with a high density of matter.

Although the temperature fluctuations are small, it provides a lot of information about the structure of the early universe. This data provides a means for testing the $\Lambda \mathrm{CDM}$ cosmological model. $\Lambda \mathrm{CDM}$ is a cosmological model that when fit to data can provide information about the energy density contributions in the form of fraction of total energy in the universe, $\Omega$. The energy density contributions are contained in the parameters $\Omega_{V}$ (dark energy density), $\Omega_{m}$ (matter density), $\Omega_{c} h^{2}$ (physical dark matter density), and $\Omega_{b} h^{2}$ (physical baryonic density). $\Omega_{b}$ (dark matter density) and $\Omega_{c}$ (baryonic matter density) can then be calculated using $\Omega_{b} h^{2}$ and $\Omega_{c} h^{2}$ respectively, along with the Hubble Constant, $H_{0}$. Using the $\Lambda$ CDM model, these parameters can then be fit to the temperature power spectrum measured by the Plank Collaboration (seen in Figure 1.4). The results of the 2015 Plank experiment are as follows: $\Omega_{V}=(0.6911 \pm 0.0062), \Omega_{m}=(0.3089 \pm 0.0062), \Omega_{b}=(0.0486 \pm 0.0010)$, $\Omega_{c}=(0.2589 \pm 0.0057)$ [1]. These results show that the energy composition of the universe is $69.11 \%$ dark energy and $30.89 \%$ matter. The remaining fraction of matter can be subset into $25.89 \%$ dark matter and $4.86 \%$ baryonic matter. This means that there is approximately $\sim 5 \times$ more dark matter in the universe then there is 
baryonic matter in the universe. Results from the Plank 2015 experiment are in close agreement with the results from the bullet cluster analysis seen above.

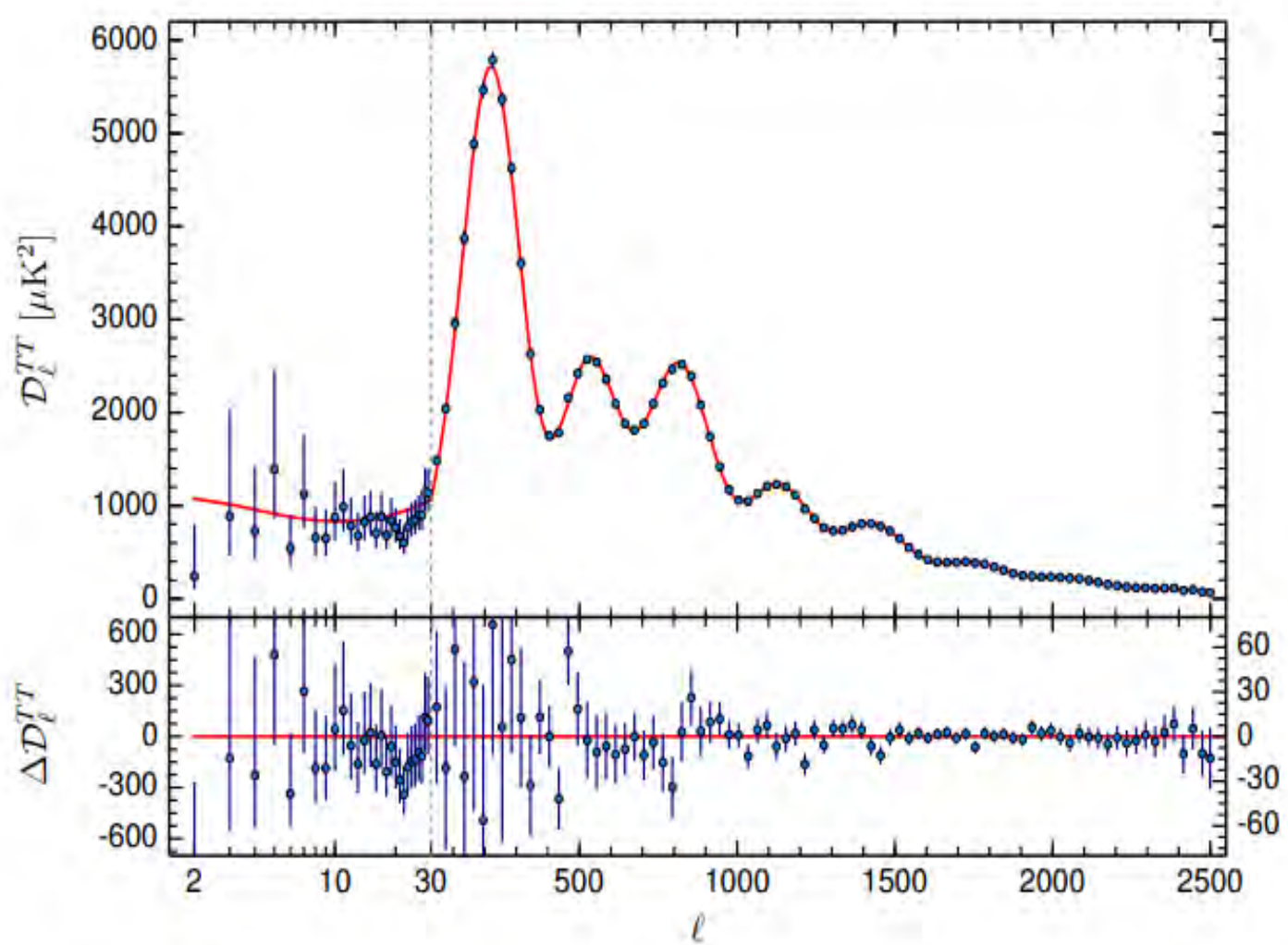

Figure 1.4: Plank 2015 temperature power spectrum $[11$. The $\Lambda$ CDM model is fit to this data, indicating that the universe is $69.11 \%$ dark energy, $25.89 \%$ dark matter, and $4.86 \%$ baryonic matter.

\subsection{Dark Matter Candidates}

Since the composition of dark matter is mostly unknown, there have been many theories that attempt to explain what it is made of and where it came from. There have been three observed characteristics of dark matter: it is very massive, it does not interact electromagnetically (with light), but does interact gravitationally. It is non-baryonic matter, meaning that it is not made up of protons and neutrons. One of the most popular model for dark matter proposes that they also interact via the weak 
force, indicating that they are Weakly Interacting Massive Particles (WIMPs). If dark matter is comprised of WIMPs they could be detectable through weak interactions on Earth (Feynman diagram of a theoretical interaction is shown in Figure 1.5) and so far such interactions have not yet been observed. Other theories to describe the composition dark matter include dark matter as axions and with the recent discovery of gravitational waves by LIGO [12], there has been an increase in research into primordial black holes as a candidate as well.

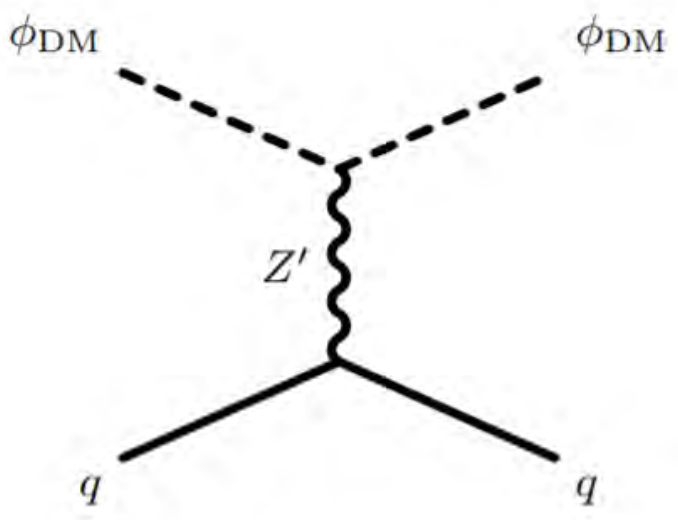

Figure 1.5: Spin independent (scalar) dark matter particle that is scattering off a nucleon/quark of the target via a Z' exchange [13].

\subsubsection{Weakly Interacting Massive Particles}

Weakly interacting massive particles, or WIMPS, are a class of non-baryonic matter that has been extensively studied as a possible way to explain dark matter. There is evidence that there was thermal production of weak scale particles (WIMPs) in the early universe that would lead to the relic density of dark matter that is seen now [14. Along with this, WIMPs also provide an explanation that could alleviate the hierarchy problem (why the weak force is $10^{24}$ times stronger then the force of gravity). The expected mass of a WIMP ranges from $\sim 5 \mathrm{GeV}-1 \mathrm{TeV}[15]$.

WIMP detection using a liquid noble gas scintillator is based off of the kinematics of a collision between a dark matter particle and target nuclei. If a WIMP collides 
and scatters off of a target nucleus depositing energy, the target nucleus would recoil producing scintillation light (discussed in Section 2.2) that can be detected. There are many ongoing experiments that are using this approach with detection targets being either liquid noble gases or other solid scintillators; or some experiments are using bubble chambers instead [16 24]. The nuclear recoil energy range that is expected for a dark matter interaction is $1 \mathrm{keV}$ to $100 \mathrm{keV}$ [25].

\subsubsection{Alternatives}

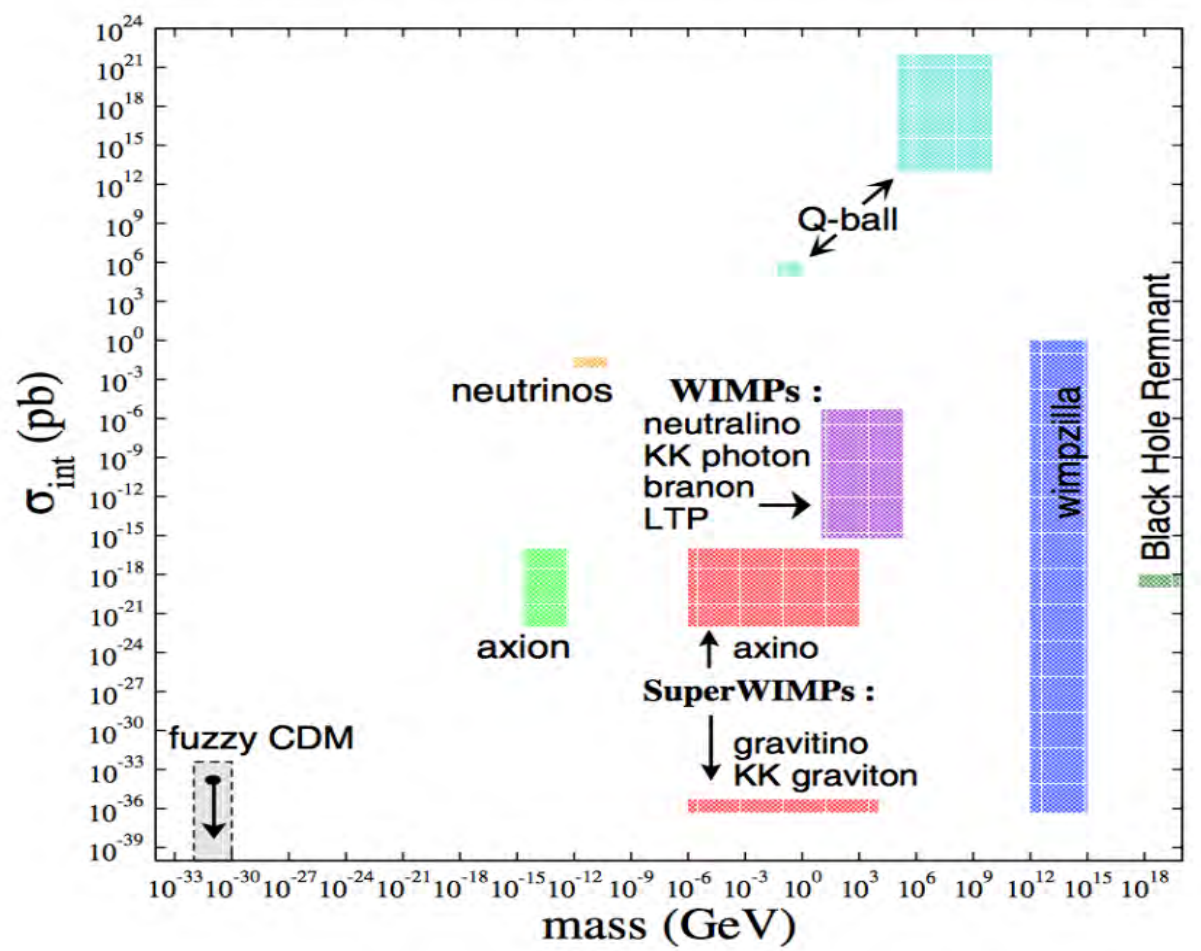

Figure 1.6: Summary of possible standard model and beyond the standard model dark matter candidates plotted in the mass and cross section regions where they are expected to be found 26]. It can be seen that WIMPs are among the heaviest possible candidates.

A second potential way to explain the composition of dark matter is axions. Axions are light, ultra-weak particles that naturally arise from a proposed solution the the Strong CP Problem [14], where C stands for charge conjugation and P stands for 
parity inversion of a quantum state. Axions are expected to be much lighter then WIMPs with masses on the order of $\mu \mathrm{eV}-\mathrm{meV}$. Currently, the Axion Dark Matter Experiment (ADMX) is the world leading axion dark matter search and has excluded the $3.3 \mu \mathrm{eV}-3.53 \mu \mathrm{eV}$ mass range [27].

Figure 1.6 is a summary of the standard model and beyond the standard model candidates that could explain dark matter. It can be seen the WIMPs are among the heaviest possible candidates and axions are among the lightest. There are also wimpzillas which are ultra heavy WIMPs, SuperWIMPs which are superweaklyinteracting and could involve particles from extra dimensions [28], neutrinos, and others.

\subsection{Current Results of Dark Matter Experiments}

There are many experiments that are looking for dark matter WIMP signals using various techniques. A summary of experimental exclusion curves produced by a number of experiments can be seen in Figure 1.7. An exclusion curve is the lower limit of a WIMP-nucleon cross-section that a given experiment is sensitive to as a function of WIMP mass. Meaning that if an experiment does not detect a WIMP signal, then any region above the exclusion curve can be ruled out for possible WIMPs. In Figure 1.7, this can be seen as the green shaded area; since only the solid line curves represent actual results (not predicted results) in this plot, the area above the most sensitive curve can be ruled out. At the bottom of Figure 1.7 there is a orange dotted line followed by a yellow shaded area, this is often referred to as the neutrino floor. The neutrino floor represents the sensitivity at which coherent neutrino scattering becomes a very large, dominant background in data and drowns out most of the other data. This would be the lowest limit for a possible WIMP discovery. 


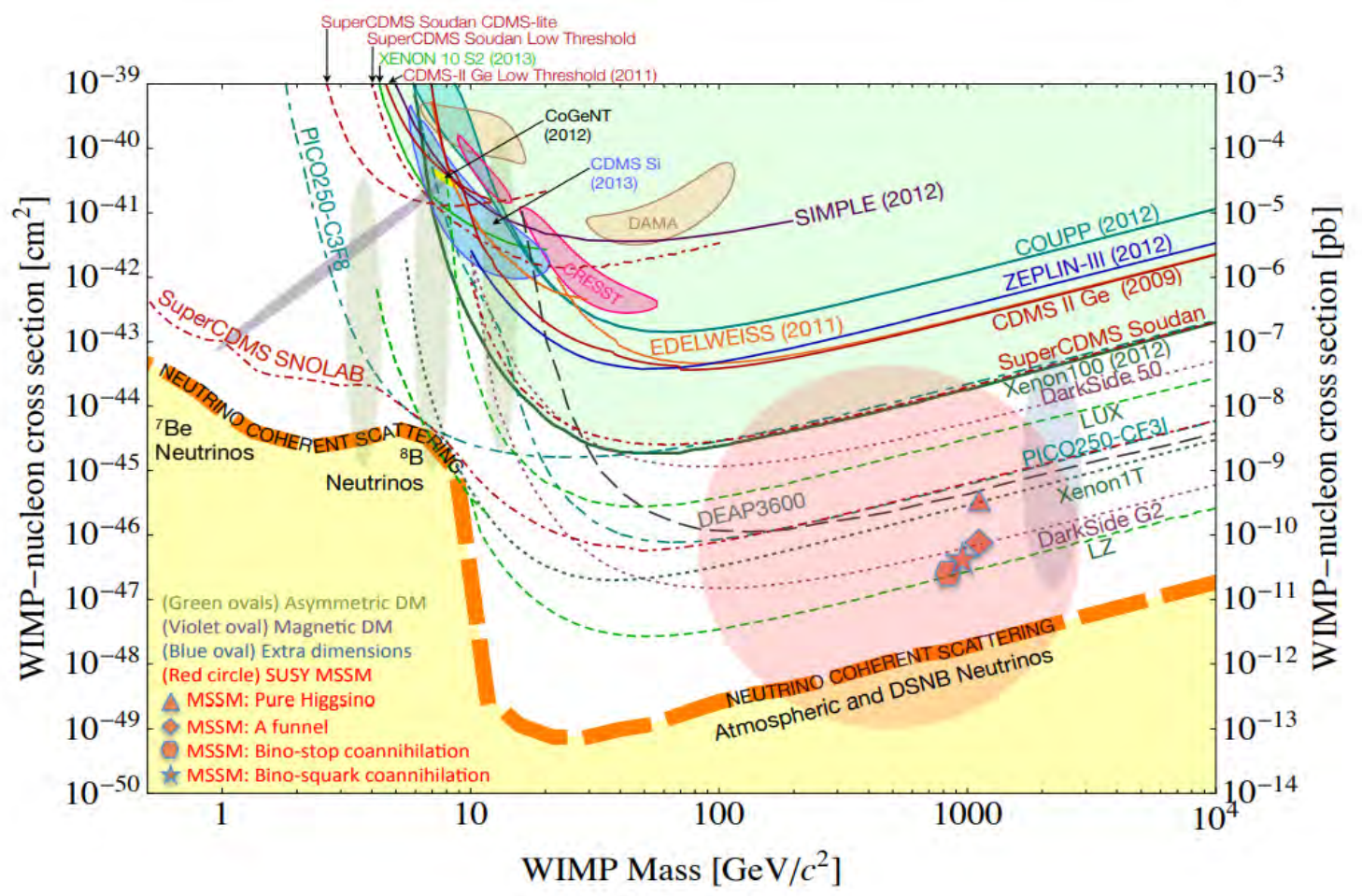

Figure 1.7: Exclusion curves from various WIMP search experiments overlaid [29]. All of the solid lines represent experiments that had results at the time this plot was made (2013) and all of the dot/dashed lines were expected results from experiments. The excluded regions of each experiment represented as the area that lies above each curve.

Presently there has been no evidence of a WIMP signal in any experiments. However, the DAMA/Nai and DAMA/LIBRA groups combined their data for analysis, resulting in an annual modulation signal that can be seen in the event rate (Figure 1.8). They have claimed that this sinusoidal modulation is due to the the earths motion through the local dark matter halo in the Milky Way [20]. Since the Milky Way is rotating through space (surrounded by a static dark matter halo) and the earth is rotating around the sun, when the earths rotation around the sun matches the rotation of the galaxy (earths maximum velocity relative to dark matter), the event rate will be at a maximum; when the earths rotation in opposite the rotation of the galaxy (earths minimum velocity relative to dark matter) the event rate will be a minimum [30]. Since the time the DAMA results were published, no other experiment 
has been able to reproduce the same signal.

\section{2-4 keV}

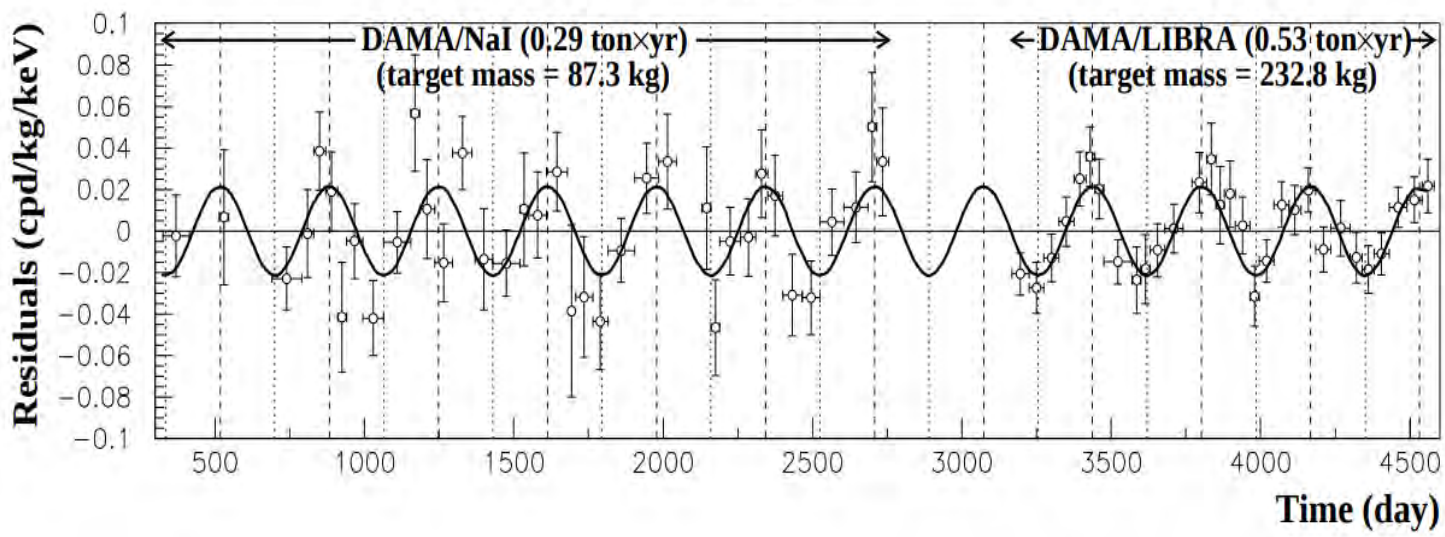

Figure 1.8: Annual modulation results presented by DAMA/Nai and DAMA/LIBRA showing a sinusoidal modulation in event rate [20]. The interpretation of this signal is that it is due the the local dark matter halo.

\subsubsection{DEAP-3600}

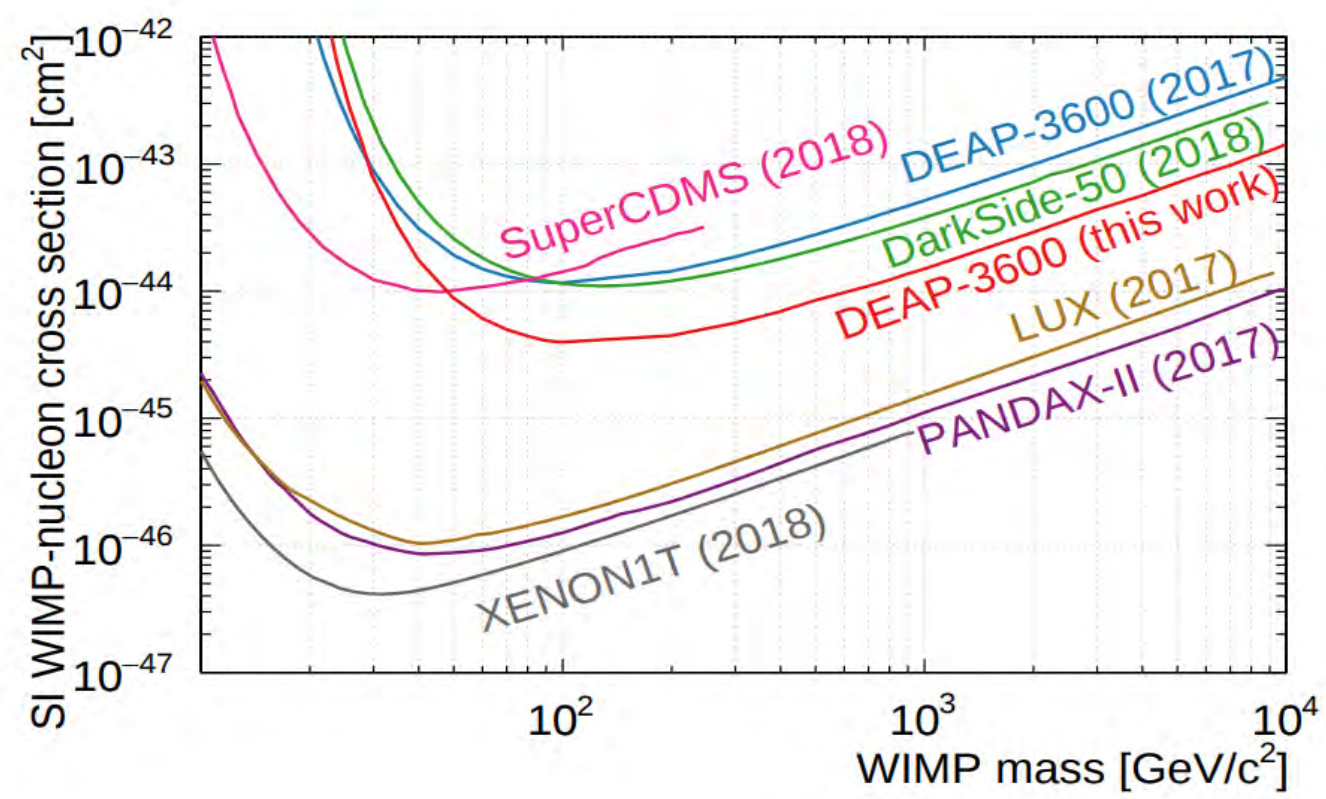

Figure 1.9: DEAP-3600 results show that it is the world leader for single-phase LAr WIMP search with a WIMP-nucleon spin independent cross section of $3.9 \times 10^{-45} \mathrm{~cm}^{2}$ to WIMPs with masses of $100 \mathrm{GeV} / \mathrm{c}^{2}[16]$. The line labelled "this work" represents the data presented in reference $[16]$. 
DEAP-3600 recently published results from a one year data set with an exposure of 231 days, seen in Figure 1.9. Based on these results is can be seen that DEAP3600 is most sensitive to $100 \mathrm{GeV} / \mathrm{c}^{2}$ WIMPS with a WIMP-nucleon cross section of $3.9 \times 10^{-45} \mathrm{~cm}^{2}[16$. Figure 1.9 shows that DEAP-3600 is the current world-leader in terms of sensitivity for single-phase liquid argon (LAr) WIMP detectors.

\subsubsection{Low Mass WIMP Search by DarkSide-50}

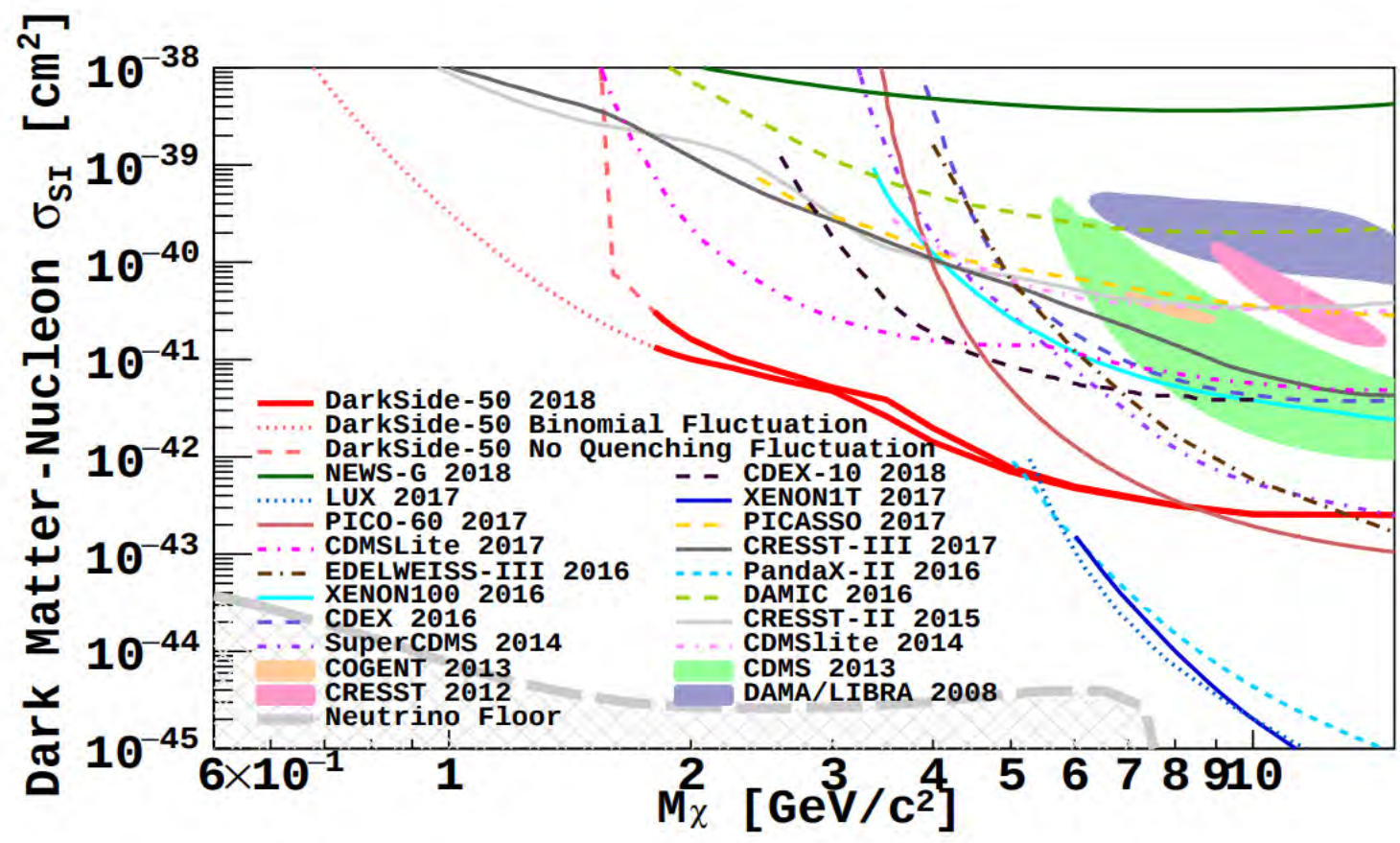

Figure 1.10: Results from DarkSide-50 low mass WIMP search that included S1+S2 and $\mathrm{S} 2$ only signals [31]. A WIMP mass lower limit of $1.8 \mathrm{GeV} / \mathrm{c}^{2}$ was achieved.

DarkSide-50 is a two-phase argon time projection chamber (TPC) detector that utilizes low radioactivity underground argon as its target mass in an attempt to detect dark matter [32]. Since there are two phases of argon in the detector, there are two signals that are observed; a LAr scintillation signal called S1 and a GAr scintillation signal due to the electric field of the TPC called S2 [32]. The standard DarkSide-50 analysis requires that there be both a S1 and S2 signal to be produced for an event 
to be included [31]. However, DarkSide-50 performed an analysis that allowed for the inclusion of events that produce S1 and S2 signals (standard analysis method) as well as events that only produce S2 signals. Allowing S2 only signals into the data set allowed the detector to be run at a lower energy threshold, which allowed for a low mass WIMP search [31.

The results of of the DarkSide-50 low mass WIMP search can be seen in Figure 1.10. It can be seen that DarkSide-50 was successful in expanding the sensitivity of the detector down to lower WIMP masses. A lower limit on the WIMP mass of $1.8 \mathrm{GeV} / \mathrm{c}^{2}$ was achieved in this analysis 31 . Due to the divergence of the Binomial Fluctuations line and No Quenching Fluctuation line below $1.8 \mathrm{GeV} / \mathrm{c}^{2}$, no claims where made about this region and future work must be pursued [31]. 


\section{Chapter 2}

\section{DEAP-3600}

DEAP-3600 is a single-phase liquid argon (LAr) detector that is searching for WIMP signals that can be attributed to the detection of dark matter. The DEAP collaboration was first formed with the DEAP-1 experiment, which be gain operation in 2006. DEAP-1 was a a prototype to DEAP-3600; it contained 7kg of LAr and two photo-multiplier tubes (PMTs). The purpose of DEAP-1 was to prove that pulseshaped discrimination (PSD) can distinguish electronic recoils from nuclear recoils. Since the decommissioning of DEAP-1 in 2007, it has been replaced with a much larger design; DEAP-3600. DEAP-3600 is currently being housed at the SNOLAB underground laboratory in the Creighton Mine in Lively, Ontario. This chapter will provide an overview of DEAP-3600 as well as the facilities where it is housed.

\subsubsection{SNOLAB Underground Research Facility}

SNOLAB was created as a location to house the Sudbury Neutrino Observatory (SNO) experiment in the mid 1990's. With the SNO experiment concluding in late

2006 the facility has been vastly expanded with $53000 \mathrm{ft}^{2}$ of clean space underground (seen in Figure 2.1) and a $34000 \mathrm{ft}^{2}$ building above ground. Along with this expansion came many new experiments, although a focus on particle physics remained, 
geophysics and bioinformatics are also being conducted.

The DEAP-3600 experiment is housed in Cube Hall, the area highlighted on the top left of Figure 2.1. The Cube Hall itself has an area of $3000 \mathrm{ft}^{2}$ and a height of $65 \mathrm{ft}$ with a deck $50 \mathrm{ft}$ above the floor. This deck sits above the DEAP-3600 experiment and holds many of the electronics and instrumentation required to run the experiment. The DEAP-3600 experiment shares this space with the miniCLEAN experiment 33].

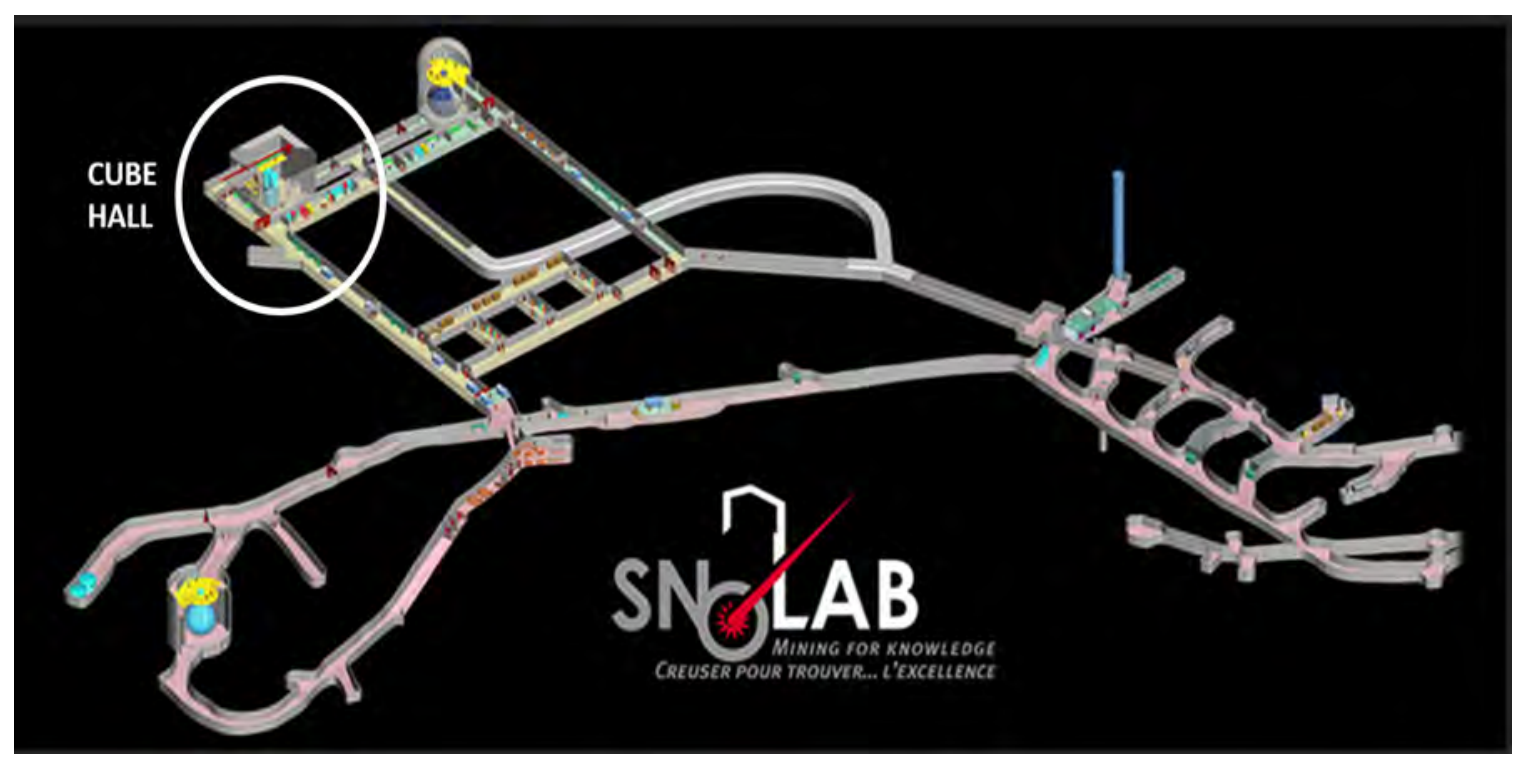

Figure 2.1: A map of the underground portion of the SNOLAB facilities. DEAP3600 resides in the Cube Hall, highlighted in the top left corner [34].

In order to conduct high sensitivity experiments, the researchers must ensure that the environment surrounding the detector has very low level of background radiation present. Many experiments that are searching for very rare events, such as a dark matter (or WIMP) interaction, locate their detectors far underground to mitigate cosmic radiation backgrounds. This is the reason why SNOLAB was chosen as the operating location of the DEAP-3600 experiment. SNOLAB is located on level 6800 of the Creighton Mine (where level 6800 means 6800 feet below ground, or $\sim 2 \mathrm{~km}$ ). Having $\sim 2 \mathrm{~km}$ of norite rock between the surface of earth and the experiment is an effective way to filter out cosmic radiation and achieve the ultra-low 
background environment required to carry out a dark matter detection experiment. The total measured cosmic ray muon flux in SNOLAB has been measured to be $0.27 \mu / \mathrm{m}^{2} / \mathrm{d}[35]$. Most other backgrounds in SNOLAB are due to neutrons from the surrounding rock as well as gamma radiation from the rock and radon gas in the air. Neutron backgrounds are especially important to understand when conducing a dark matter search as they can reproduce WIMP like signals in the detector. The measured neutron flux from the rock is $(4145 \pm 155)$ neutrons $/ \mathrm{m}^{2} / \mathrm{d}[35]$. The measured gammaray flux that is produced in the rock can be seen in Table 2.1.

\begin{tabular}{|c|c|}
\hline $\begin{array}{c}\text { Energy } \\
{[\mathrm{MeV}]}\end{array}$ & $\begin{array}{c}\text { Flux } \\
{\left[\gamma / \mathrm{m}^{2} / \text { day }\right]}\end{array}$ \\
\hline $4.5-5$ & $510 \pm 220$ \\
$5-7$ & $360 \pm 220$ \\
$>7$ & $180 \pm 90$ \\
$>8$ & $<20$ \\
\hline
\end{tabular}

Table 2.1: Gamma radiation from norite rock in SNOLAB [35].

\subsection{The Detector}

The DEAP-3600 detector consists of an acrylic sphere (often called the acrylic vessel $(\mathrm{AV}))$ that is $5 \mathrm{~cm}$ thick and has an inner radius of $85 \mathrm{~cm}$. The AV is capable of holding up to $3600 \mathrm{~kg}$ of LAr, but currently holds $(3279 \pm 97) \mathrm{kg}$ of LAr (See Appendix A). There are 255 acrylic cylindrical light guides (LGs) that are $45 \mathrm{~cm}$ long and bonded to the outer surface of the AV. These LGs couple the AV to 255 Hamamatsu R5912-HQE (high quantum efficiency) photo-multiplier tubes (PMTs) that view the AV. These PMTs have a $22 \%$ quantum efficiency at a wavelength of $390 \mathrm{~nm}$ [36]. The area between the LGs is filled with high density polyethylene and polystyrene filler blocks. The filler blocks serve as additional shielding to incoming neutrons from the surrounding rock as well as structural support for the LGs. The 
inner surface of the AV has been coated with a wavelength-shifter called 1,1,4,4tetraphenyl-1,3-butadiene (TPB); the coating is $3 \mu \mathrm{m}$ thick. The purpose of the TPB is to shift the vacuum ultra-violet (VUV) light emitted from argon scintillation into a detectable wavelength at $\sim 420 \mathrm{~nm}$, near the peak efficiency of the PMTs [37]. With all of these components assembled, the detector is then enclosed inside a pressurized stainless steel shell. On the outside of the stainless steel shell are 48 Hamamatsu R1408 PMTs that serve as a muon veto system (discussed in Section 2.5). The stainless steel shell is then immersed inside of a water tank that is $7.8 \mathrm{~m}$ in diameter and is filled with ultra-pure water. This water tank acts as both the other part of the muon veto system as well as shielding from neutrons that are emitted by the surrounding rock. A schematic diagram of the DEAP-3600 detector can be seen in Figure 2.3 and various phases of the detector construction can be seen in Figure 2.2 .

During the construction of the detector there was a great deal of care taken in order to ensure that any background that could duplicate a WIMP signal would be kept to an absolute minimum. Acrylic was chosen as the AV material due to the ease of manufacturing which allows for control over the introduction of radioactive materials during the manufacturing process. As mentioned in Section 2.0.1 the air in SNOLAB contains radon which can be deposited on any part of the detector during construction. To mitigate this as much as possible, exposure to the lab air was kept to a minimum during the assembly, and the inner surface of the AV was resurfaced before completion to remove any potentially unwanted contaminants that would come into contact with the LAr. 


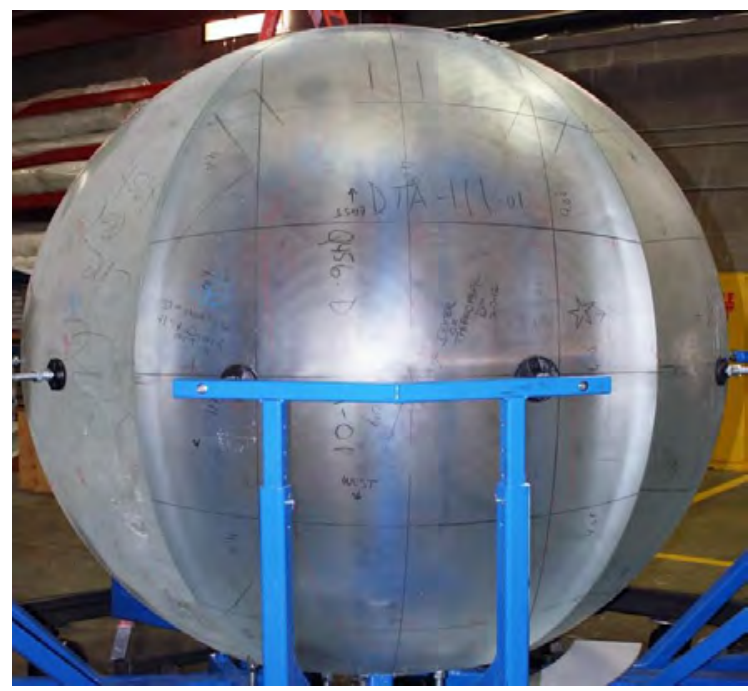

(a) Four quarters of the AV being bonded.

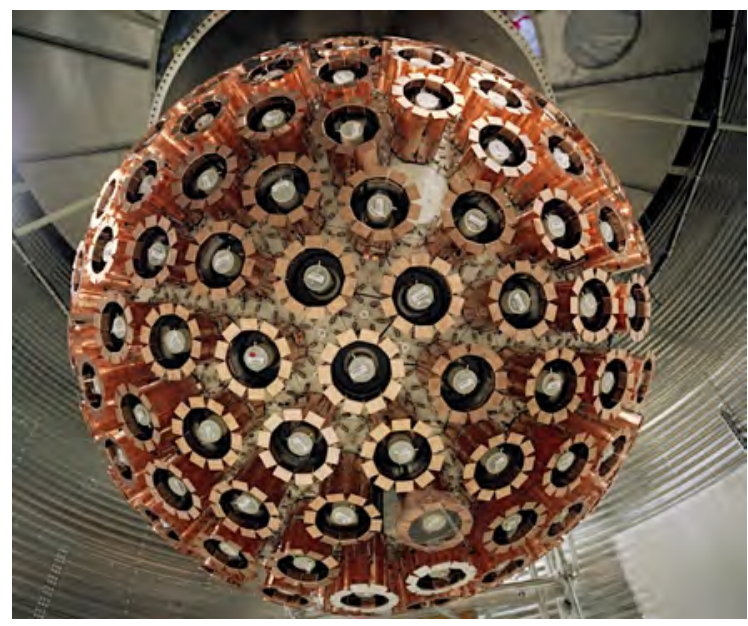

(c) AV with all the PMTs mounted.

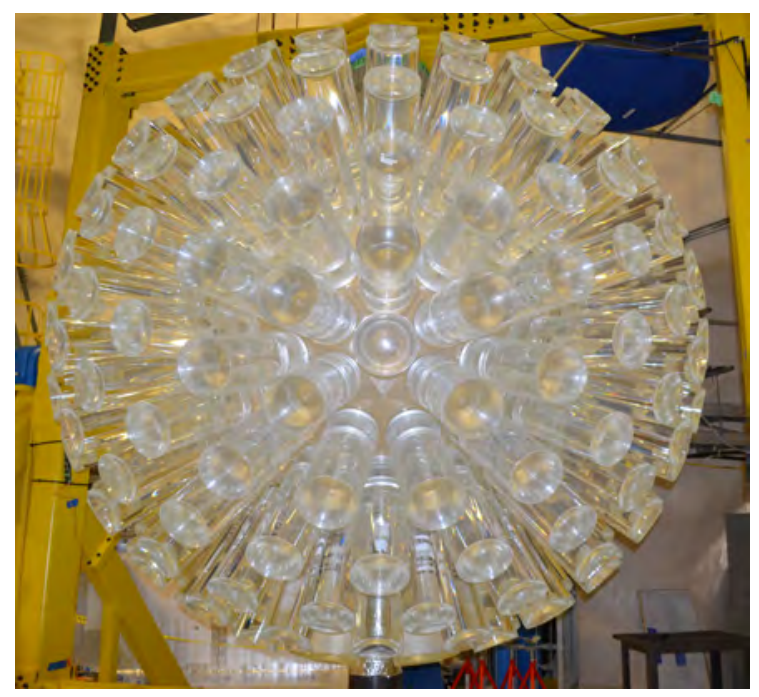

(b) AV with all the light guides bonded.

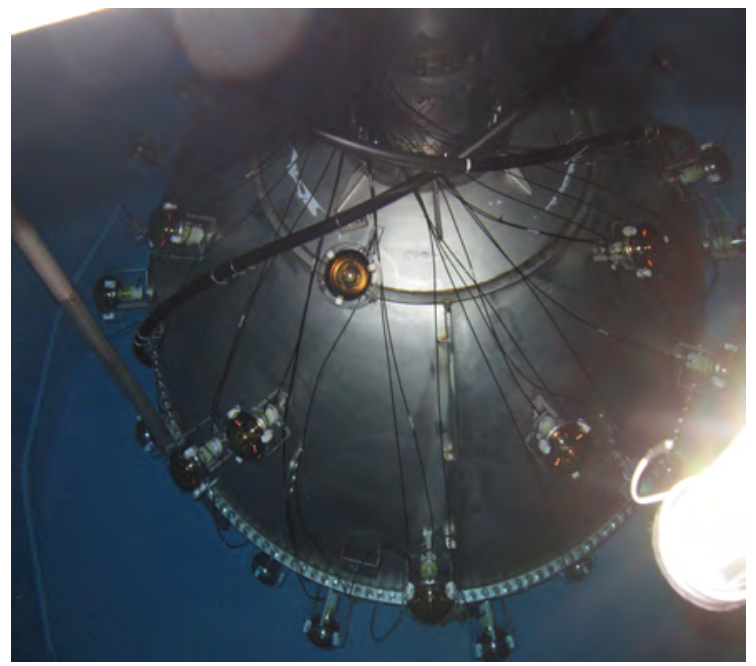

(d) Competed detector submerged in the water tank.

Figure 2.2: Photos of various stages of construction of the DEAP-3600 detector. 


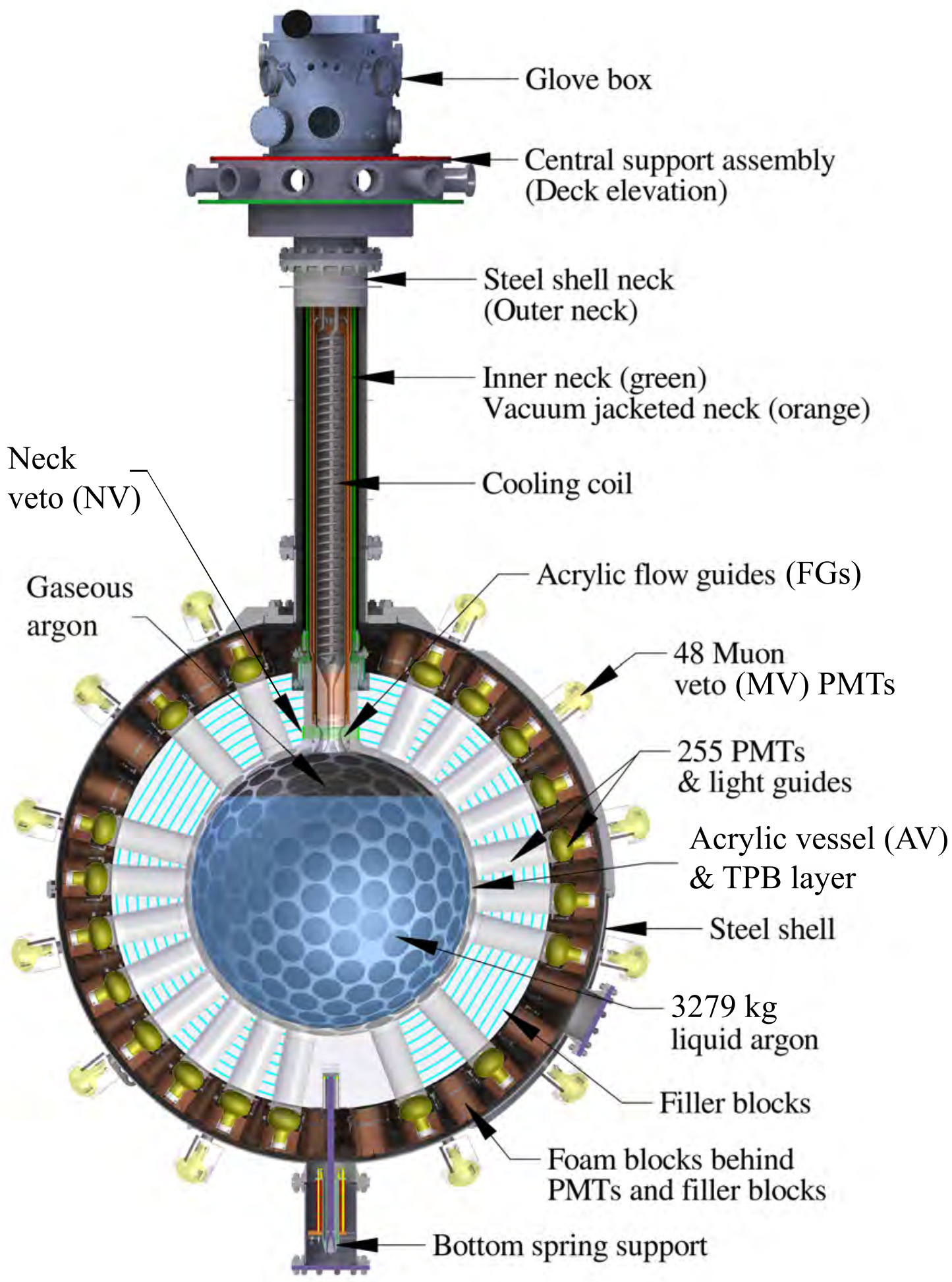

Figure 2.3: Schematic diagram of the DEAP-3600 detector. 


\subsection{Scintillation in Liquid Argon}

When an incoming particle enters the LAr in the detector, it will scatter off of either an electron or nucleus depositing energy. This energy will cause the particle that is struck (electron or nucleus) to recoil, leaving the atom in an excited state. As the particle recoils through the target volume, the energy gained from the collision will be dissipated through the excitation or ionization of surrounding atoms, as well as heat when the recoiling particle no longer has enough energy to excite surrounding atoms [38]. Excited atoms in the LAr, called excitons, as well as ions (ionized particles) can form bonds with ground state atoms called dimers.

Scintillation light is produced by excited dimers (called excimers) in the LAr decaying to ground state. The scintillation light has a wavelength of $126.8 \mathrm{~nm}$ (at $85 \mathrm{~K})$ [39. However, the decay channels for excimers that are created through the excitation of atoms and ionization of atoms are not the same. The decay of an excimer formed with an exciton can be seen in Equation 2.2 and the decay of an excimer formed through ionization can be seen in Equation 2.3 [40].

$$
\begin{gathered}
\chi+A r \longrightarrow A r^{*}+\chi^{\prime} \\
A r^{*}+A r \longrightarrow A r_{2}^{*} \\
A r_{2}^{*} \longrightarrow 2 A r+\gamma .
\end{gathered}
$$

The lowest energy excimers in liquid noble gases correspond to a singlet state $\left({ }^{1} \Sigma_{u}^{+}\right)$and a triplet spin state $\left({ }^{3} \Sigma_{u}^{+}\right)$. The ground state $\left({ }^{1} \Sigma_{g}^{+}\right)$spin of LAr is $S=0$. Since the singlet spin state also has spin $S=0$, then it can freely decay radiatively to ground state. The triplet spin state has total spin $S=1$ so this decay is forbidden due to conservation of angular momentum and the selection rule, $\Delta S=0$ [41]. However, this is well known for hydrogen-like atoms but does not well represent heavy atoms, 
such as argon. Because of this, the triplet state can indeed decay radiatively to the ground state, albeit at a suppressed rate 41.

$$
\begin{gathered}
\chi+A r \longrightarrow A r^{+}+\chi^{\prime}+e^{-} \\
A r^{+}+A r \longrightarrow A r_{2}^{+} \\
A r_{2}^{+}+e^{-} \longrightarrow A r^{* *}+A r \\
A r^{* *} \longrightarrow A r^{*}+\text { heat } \\
A r^{*}+A r \longrightarrow A r_{2}^{*} .
\end{gathered}
$$

The decay lifetime of the triplet state will much longer the decay life time of the singlet state due to the suppressed decay rate. The singlet state decay time is $\tau<6.2 \mathrm{~ns}$ and the triplet decay time is $\tau=(1300 \pm 60)$ ns $[39]$. This timing difference is the driving force behind the analysis technique called pulse-shape discrimination (PSD). Experimenters are able to collected the scintillation light that is given off by decaying excited particles and based on the decay time of the event, the type of particle interaction can be determined.

\subsection{Hardware Trigger System}

The main component of the trigger system in DEAP-3600 is the data acquisition system (DAQ). The DAQ is connected to all 255 inner detector PMTs, the 48 muon veto PMTs, as well as 4 neck PMTs. The DAQ contains 27 signal conditioning boards (SCBs), each SCB has 12 channels allowing all the PMTs to have its own channel. The 22 SCBs connected to the inner PMTs are connected to a total of 36 digitizers, 32 V1720s for evaluating fast pulses and 4 V1740s for evaluating slow pulses. The 22 inner PMT SCBs are also connected the digitizer and trigger module (DTM). 
The four muon veto SCBs are connected to their own V1740 digitizer and the neck SCB to its own V1720 digitizer. All the the digitizers are then connected to readout computers. A schematic diagram of the DAQ can be seen in Figure 2.4

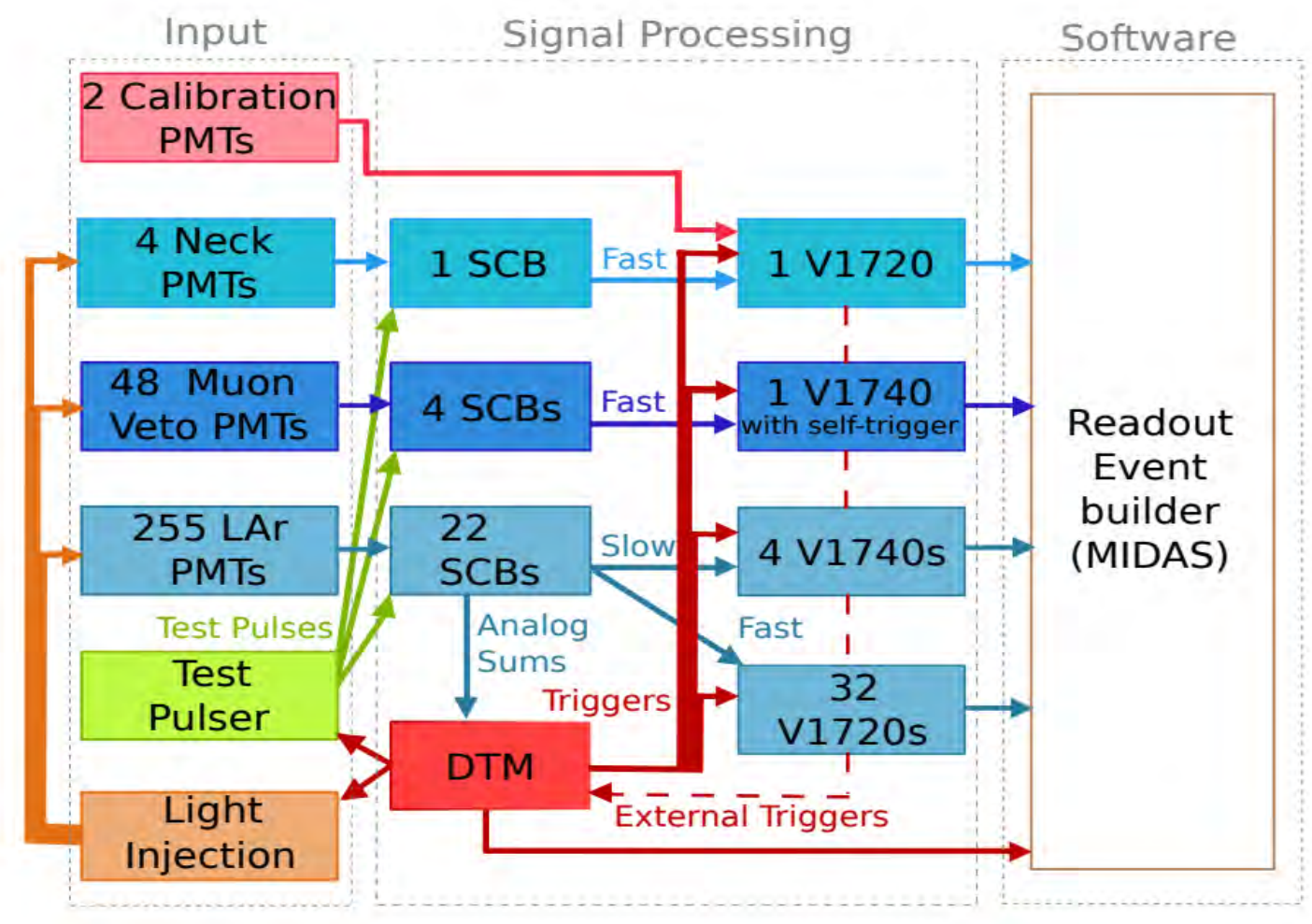

Figure 2.4: Schematic diagram of the data acquisition system used by DEAP-3600.

The DAQ is continuously monitoring the detector by performing rolling integrals over the first $150 \mathrm{~ns}$ of a pulse (narrow window) and over the first $10 \mu \mathrm{s}$ of a pulse (wide window). The DAQ will trigger if the charge integral is greater then the set threshold (for standard physics data this is $1000 \mathrm{ADC}$ ). "ADC" represents an analogto-digital converter, which is used to the convert a continuous analog voltage signal into a discrete digital signal. The digitizers operate with a 12-bit precision, meaning that there are 4096 discrete voltage values in ADC units. If the trigger is classified as a periodic trigger (discussed in Section 3.1) then that DAQ is forced to trigger with no threshold. If the conditions are met such that the DAQ triggers, a $16 \mu$ s long waveform will be recorded. $2.5 \mu$ s from before the time of the trigger and $13.5 \mu$ s after 
the time of the trigger. This information is then sent to the output computers for further processing. The event information is then saved as well as the information about the nature of the trigger, which is produced by the DTM.

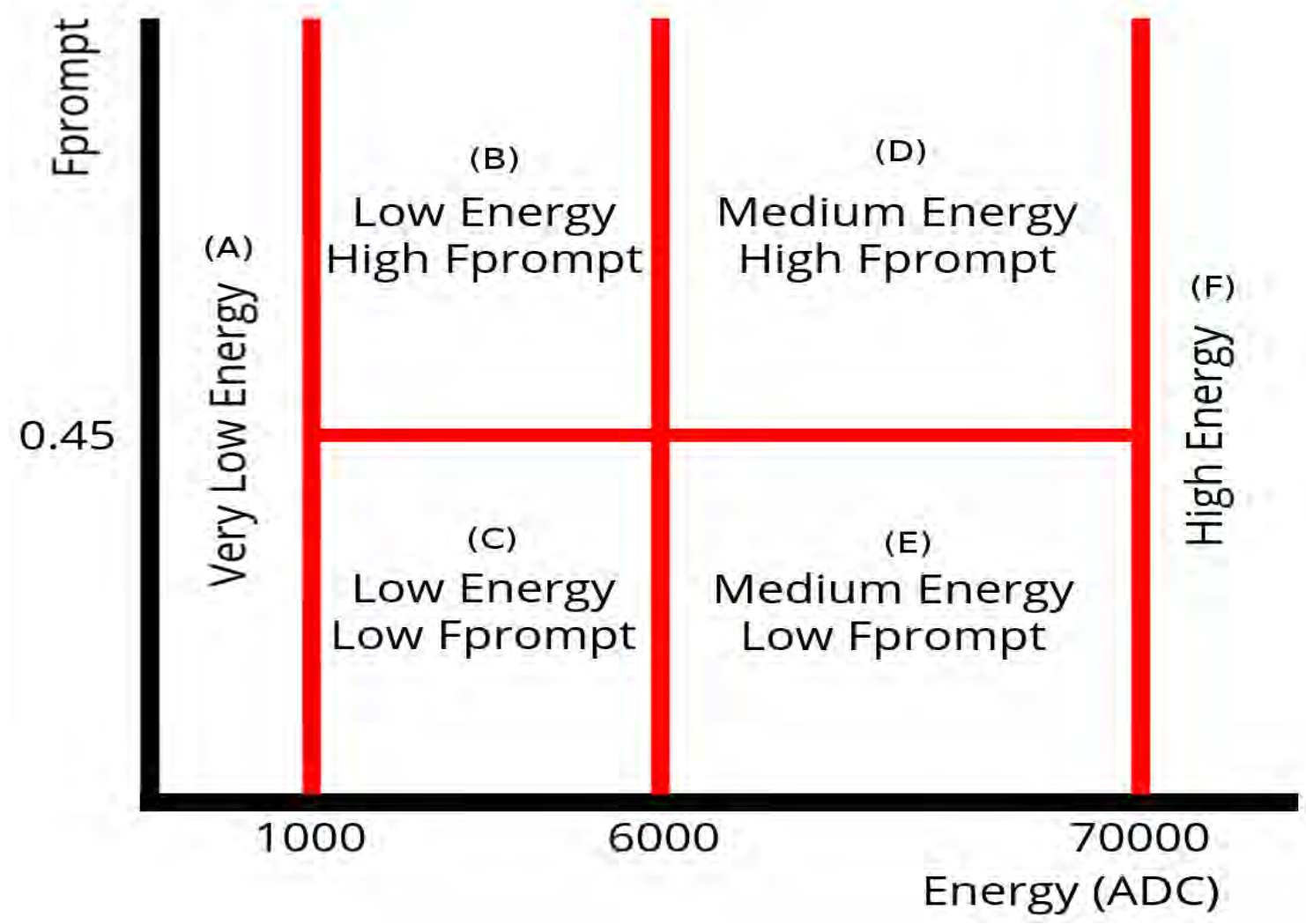

Figure 2.5: Trigger regions for which the data taken by DEAP-3600 can be saved to; where energy is the prompt energy of the event. A description of $\mathrm{F}_{\mathrm{prompt}}$ can be found in Section 4.1.4.

Data in DEAP-3600 is first characterized based on the integrated energy of the narrow window as well as a PSD parameter known as $\mathrm{F}_{\text {prompt }}$ (discussed in Section 4.1.4. There are six regions for which an event can be characterized; a diagram of the six regions can be seen in Figure 2.5. Region E is of particular importance in DEAP-3600 and is referred to as the prescaled region. Region E lies within the electron recoil band (ERB) where ${ }^{39} \mathrm{Ar}$ decays dominate the energy spectrum; due to the high rate of these decays $(\sim 3000 \mathrm{~Hz})$ it is unreasonable to keep all of this data. To save disk space, the solution was to only save information from 1 of every 100 
events within this region. The regions that will be used most in this work are regions A, B, and C. However, all the regions may be referred to in order to draw comparisons to standard physics data or in order to understand where some populations of low energy events originate from.

\subsubsection{Trigger Efficiency}

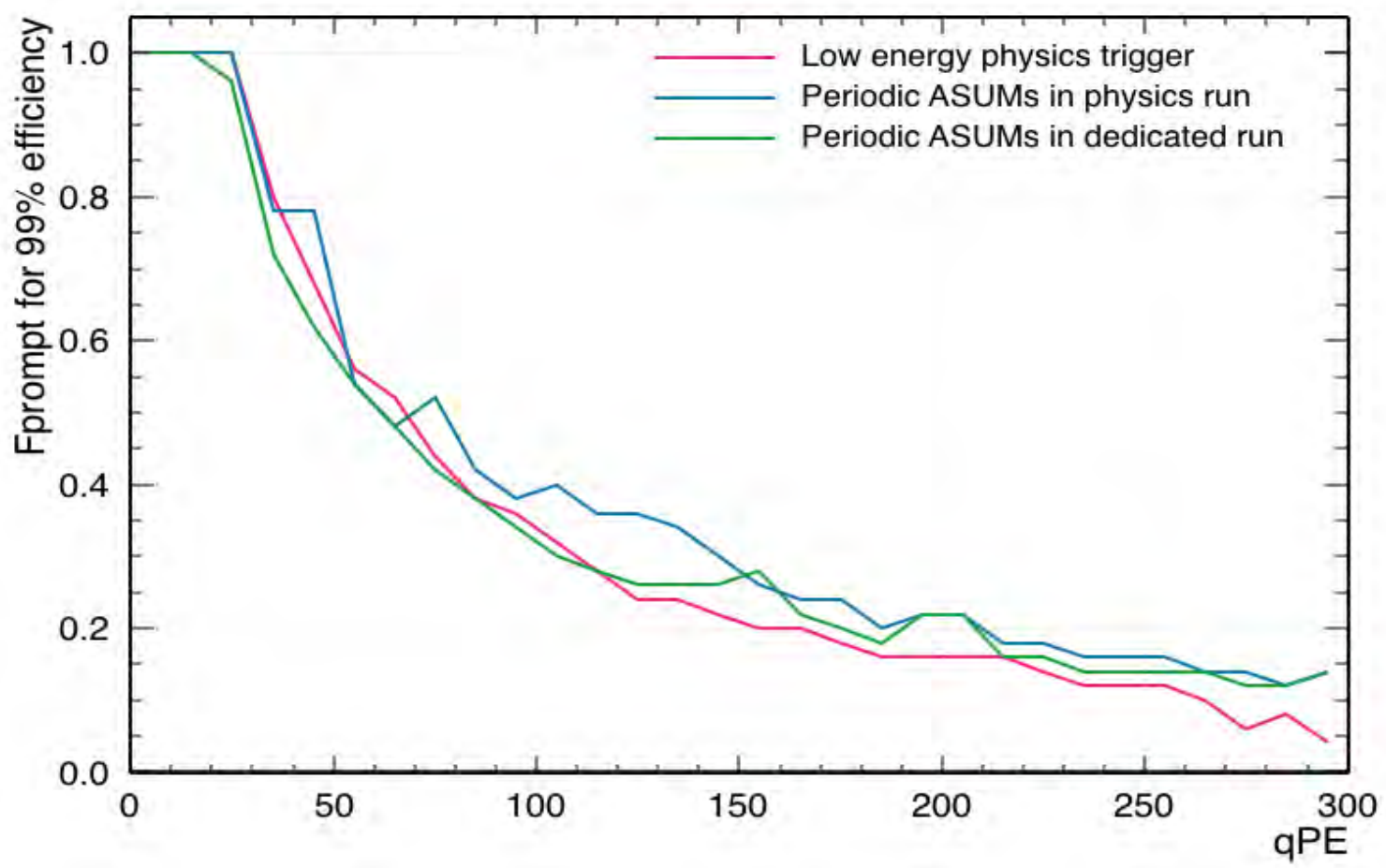

Figure 2.6: The DAQ trigger efficiency shown in PSD-space (discussed in Section 2.4. Where the solid pink line represents the cut off for $99 \%$ trigger efficiency.

Due to instrumental limitations, the trigger efficiency is not $100 \%$ for all energies. As a consequence the DAQ may miss some signals from primarily lower energy events. The DEAP-3600 trigger efficiency can be seen in Figure 2.6. The curve in Figure 2.6 shows that there is a definite $\mathrm{F}_{\text {prompt }}$ dependence to the trigger efficiency. This figure can be interpreted in the following way; it can be said that the standard physics trigger is $99 \%$ efficient above $\sim 200 \mathrm{qPE}$ (the qPE variable is an estimate of the total 
measured number of photoelectrons (PE) that are produced by an event, discussed in Section 4.1.3 and above $\mathrm{F}_{\text {prompt }}=0.2$. As a results of the $\mathrm{F}_{\text {prompt }}$ dependence of the trigger efficiency, the detector becomes $>99 \%$ efficient at detecting different kinds of particles at different energies.

\subsection{Pulse-Shape Discrimination}

Pulse-shape discrimination (PSD) arises due to the difference in timing that it takes for photons to arrive at the PMTs. As mentioned in Section 2.2 the singlet and triplet decay times of excimers in LAr are very different. Both excitons and ions form both triplet and singlet state excimers, but through different channels. The ionization channel involves the introduction of a new electron into an atom that has a spin state that is independent of the spin state of the atom it recombines with. Meaning that it is likely that the spin states of the introduced electron and electron that was ionized from the atom that it recombines with are not the same, indicating that the ionization channel is more likely to form a triplet state excimer. However, an exciton is formed with the promotion of an electron into the next energy level, so this electron and atom will maintain their original spin states. This indicates that the exciton channel is more likely to form a singlet state excimer. Since there is a higher probability for an electron to be ionized then a nucleus (due to the large amount of energy required to ionize a nucleus), electronic recoils form more triplet state excimers than nuclear recoils. The probability of an electron recoil forming an excimer through the excitation channel is $21 \%$, 42 , and the probability of a nuclear recoil forming an excimer through the excitation channel is $\sim 100 \%$ [4].

PSD provides a powerful method to distinguish between relatively low energy electronic events compared to higher energy nuclear recoils. Figure 2.7 shows the difference between the waveforms produced by a nuclear recoil and electronic recoil. 
There is more late light in the electronic recoil waveform, meaning that there are more triplet state excimers generated in this interaction.

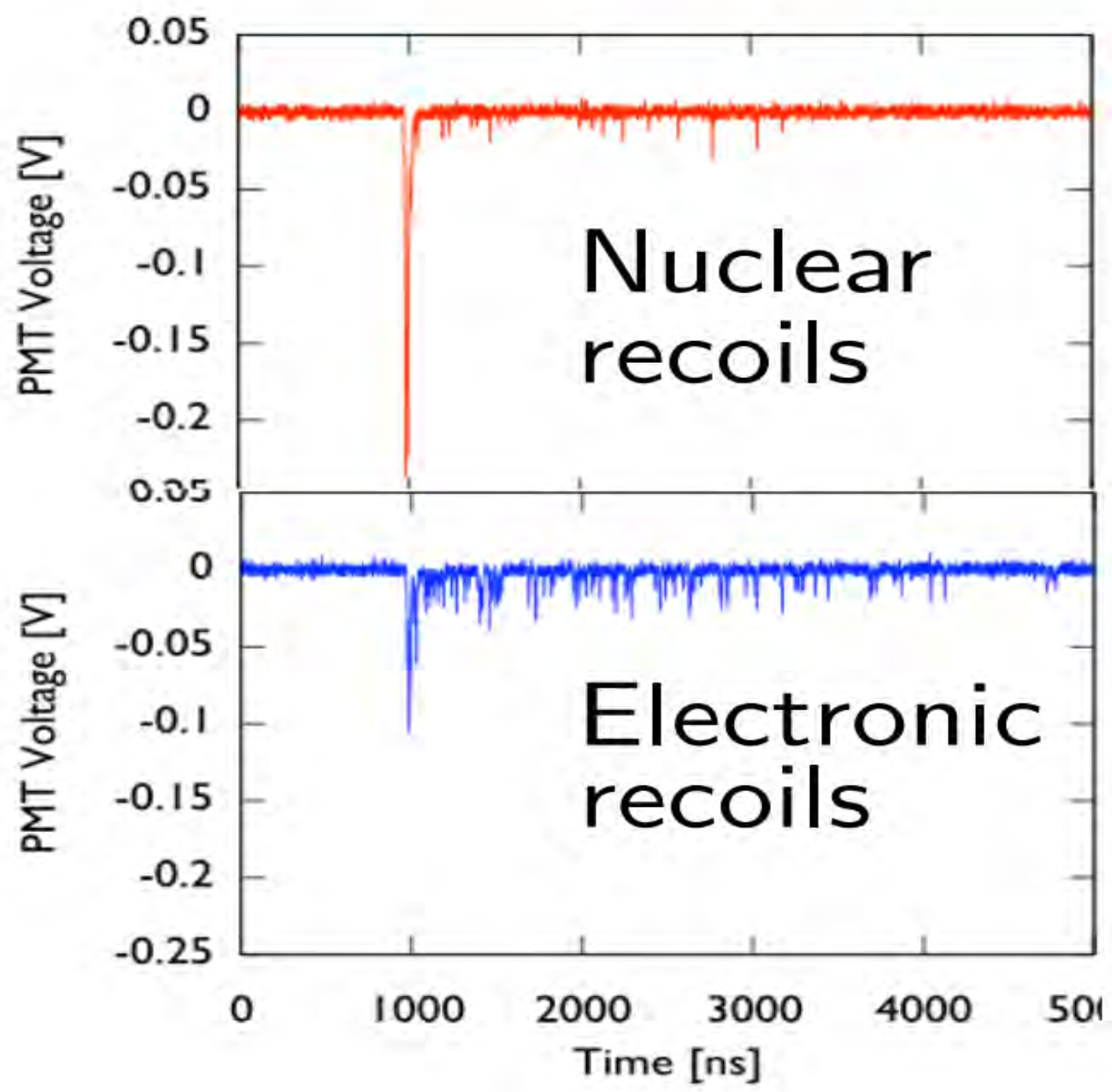

Figure 2.7: Difference between an electronic and nuclear recoil in data. It can be seen that the electronic recoil waveform contains more late light then the nuclear recoils. This indicates that there is a larger fraction of triplet state excimers in electronic recoils than nuclear recoils.

When looking at the PSD parameter $F_{\text {prompt }}$ (discussed in Section 4.1.4) these timing differences between nuclear recoil and electronic recoils give rise to two distinct bands. In Figure 2.8 the electron recoil band $(\mathrm{ERB})$ can bee seen at $\mathrm{F}_{\text {prompt }} \sim 0.3$ (orange band) and the nuclear recoil band $(\mathrm{NRB})$ can be seen at $\mathrm{F}_{\text {prompt }} \sim 0.7$ (purple 
band). Though, the clear distinction between these bands to begins to break down at low energies.

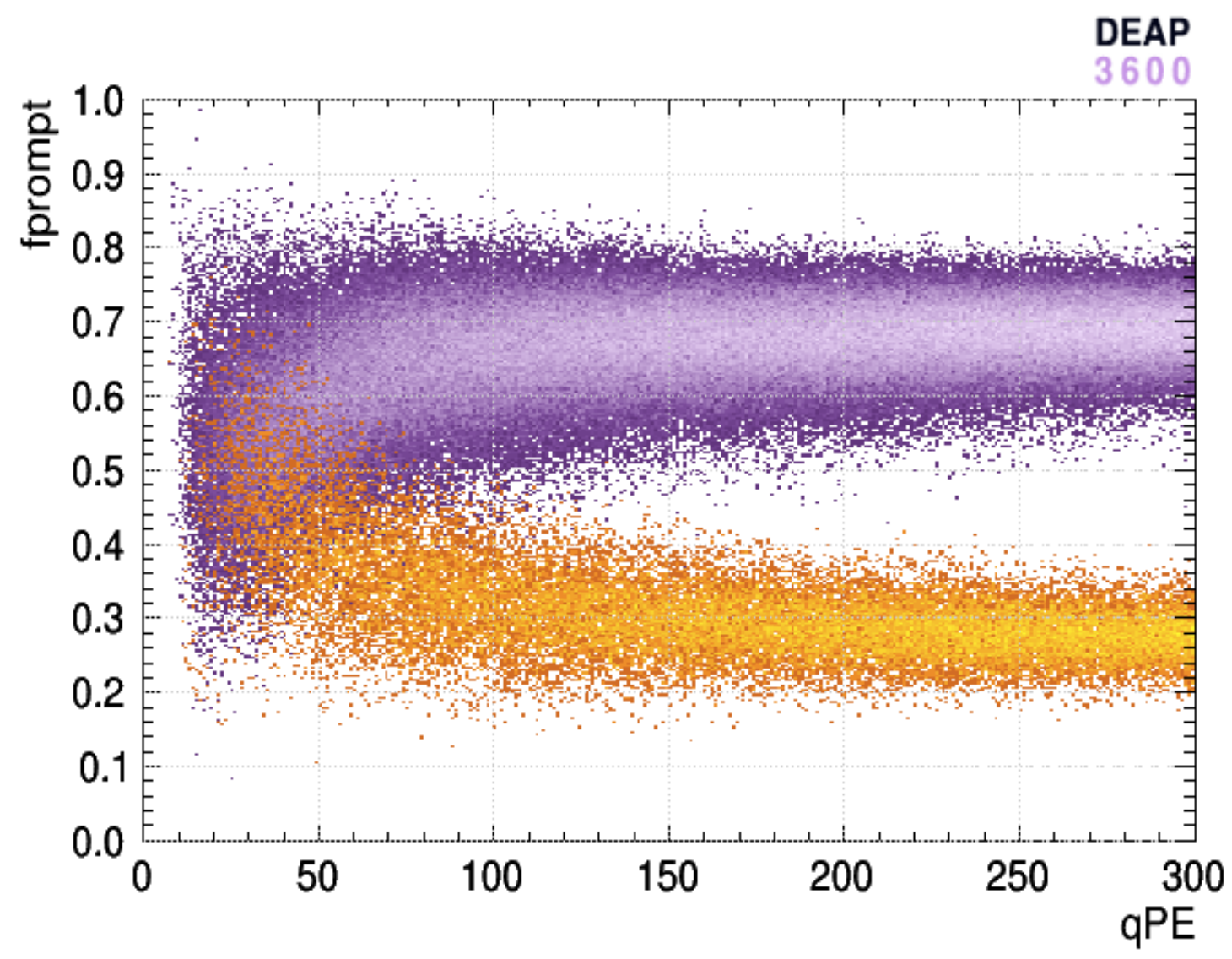

Figure 2.8: Difference between and electronic recoils band (ERB), the orange band, and nuclear recoil band (NRB), the purple band based off on Monte Carlo data. Based on the definition of $\mathrm{F}_{\text {prompt }}$ (Section 4.1.4) this shows that there is more late light produced by a electronic recoil then a nuclear recoil.

\subsection{Backgrounds}

Even with all the measures taken to reduce the backgrounds as much as possible it is impossible to eliminate all the backgrounds. The largest background comes from cosmogenically produced ${ }^{39} \mathrm{Ar}$ found in atmospheric argon in the form of $\beta$-decays. Other backgrounds come from neutron radiation, $\alpha$-decays, cosmogenic muons and most importantly at low energies, Cherenkov radiation induced by thorium $\left({ }^{232} \mathrm{Th}\right)$ 
decay progeny, uranium $\left({ }^{238} \mathrm{U}\right)$ decay progeny, and potassium $\left({ }^{40} \mathrm{~K}\right)$ decays. This section will provide details about where these backgrounds come from and how each one can effect the data.

\subsection{1 ${ }^{39}$ Ar Events}

${ }^{40} \mathrm{Ar}$ is a naturally occurring isotope that makes up $\sim 1 \%$ of earth's atmosphere. The radioactive isotope ${ }^{39} \mathrm{Ar}$ is primarily produced when cosmic rays interact with ${ }^{40} \mathrm{Ar}$ in the atmosphere. However, ${ }^{39} \mathrm{Ar}$ can also be produced underground through neutron-capture with ${ }^{40} \mathrm{~K} .{ }^{39} \mathrm{Ar}$ undergoes $\beta$-decay, has a half-life of ( $\left.269 \pm 3\right)$ years and decays into ${ }^{39} \mathrm{~K}$, which is a stable isotope (shown in Equation 2.3 [44. The Q-value for this decay is $(565 \pm 5) \mathrm{keV}[45]$. The ${ }^{39} \mathrm{Ar}$ spectrum can be seen in Figure 2.9 .

$$
{ }_{18}^{39} A r \longrightarrow{ }_{19}^{39} K+e^{-}+\bar{\nu}_{e}
$$

The specific activity of ${ }^{39} \mathrm{Ar}$ in atmospheric argon in DEAP-3600 has been measured to be $(0.95 \pm 0.05) \mathrm{Bq} / \mathrm{kg}[46$. Due to the large volume of argon in the detector this becomes a very large background that has a rate of $\sim 3000 \mathrm{~Bq}$. As mentioned in Section 2.3, it is not necessary to record the waveforms of every event in the ERB because it is dominated by ${ }^{39} \mathrm{Ar}$ events. Instead only one waveform of every one hundred events is saved to the disc; this keeps the data files small and save disc space, while not loosing information. The other main reason for not saving all the events in the ${ }^{39} \mathrm{Ar}$ region is because the electronics would not be able to support the high trigger rate if pulse information for all of these events was saved. Within the DEAP3600 collaboration this is referred to as prescaling. The non-prescaled spectrum can be recovered at anytime by multiplying the prescaled region by 100, at the cost of having fewer event statistics. 


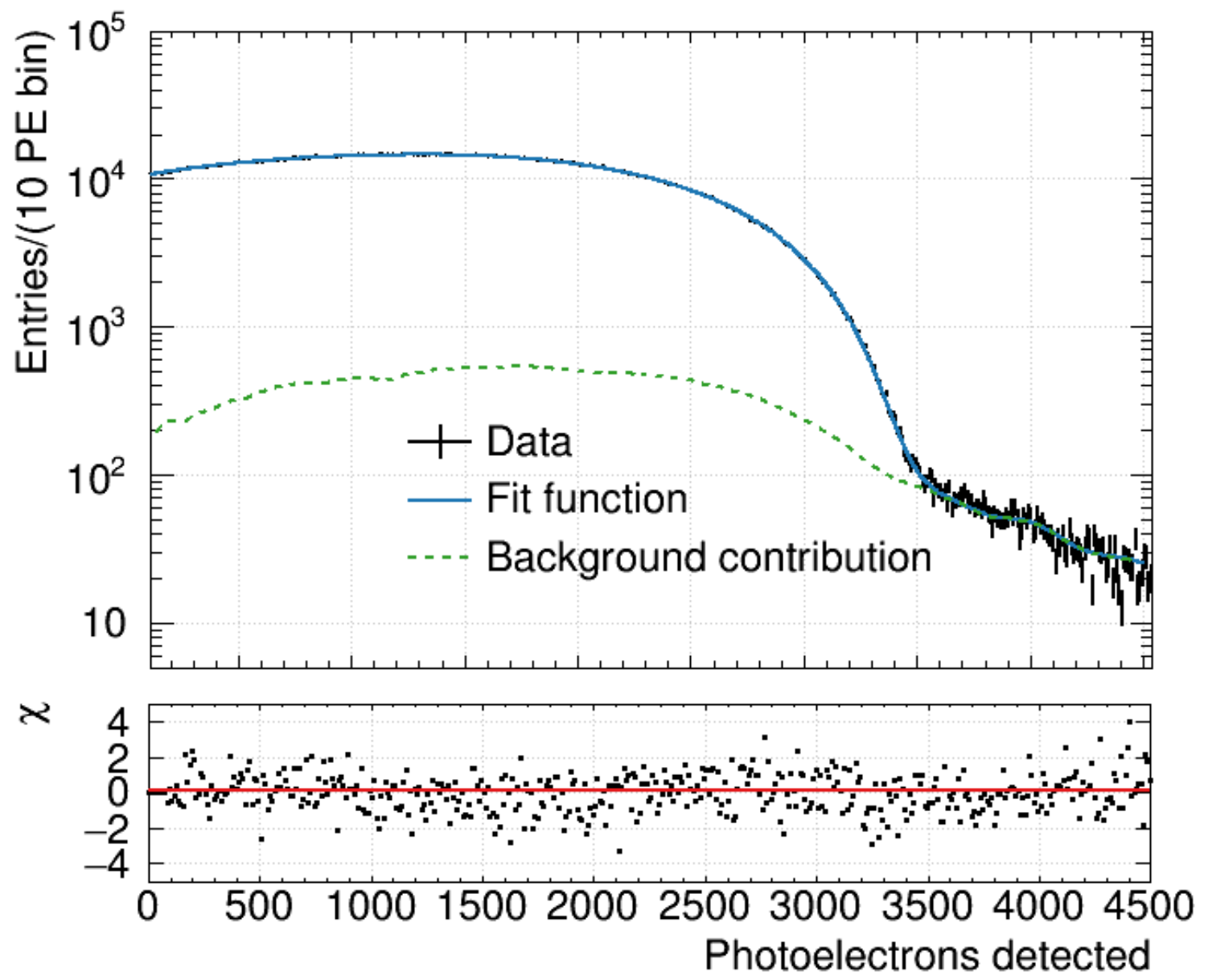

Figure 2.9: ${ }^{39} \mathrm{Ar}$ model fit to data from DEAP-3600 $[16$. There is very good agreement between the model (blue) and data (black).

\subsubsection{Cherenkov Radiation}

Cherenkov radiation is light that is produced when a charged particle passes through a dielectric material with a speed greater then the phase speed of light of the material. Since the particle is travelling faster than the speed of light, the light wave cannot propagate forward. So the light is emitted as a wave front that can be pictured as a cone peaked towards the movement of the particle. This can be created by any charged particle. However, in DEAP-3600 the main particles that can produce Cherenkov radiation are electrons and gammas, which are produced by radioactive decays. Since gammas are not charge particles, they can not produce Cherenkov ra- 
diation directly. Instead, when a gamma enters a material and Compton scatters off of an electron, the scattered electron produces Cherenkov radiation.

Although materials in DEAP are chosen such that radioactive backgrounds are reduced as much as possible, it is impossible to eliminate completely. Some of the main sources of radioactivity in DEAP-3600 are ${ }^{232} \mathrm{Th},{ }^{238} \mathrm{U}$ as well ${ }^{40} \mathrm{~K}$ in the LGs and PMTs. There are also minor contributions from ${ }^{235} \mathrm{U}$ in the PMTs, ${ }^{232} \mathrm{Th}$ from the AV acrylic, and ${ }^{60} \mathrm{Co},{ }^{238} \mathrm{U}$, and ${ }^{232} \mathrm{Th}$ from the stainless steel shell. These rates are much lower then the rates produced in the LGs and PMTs.

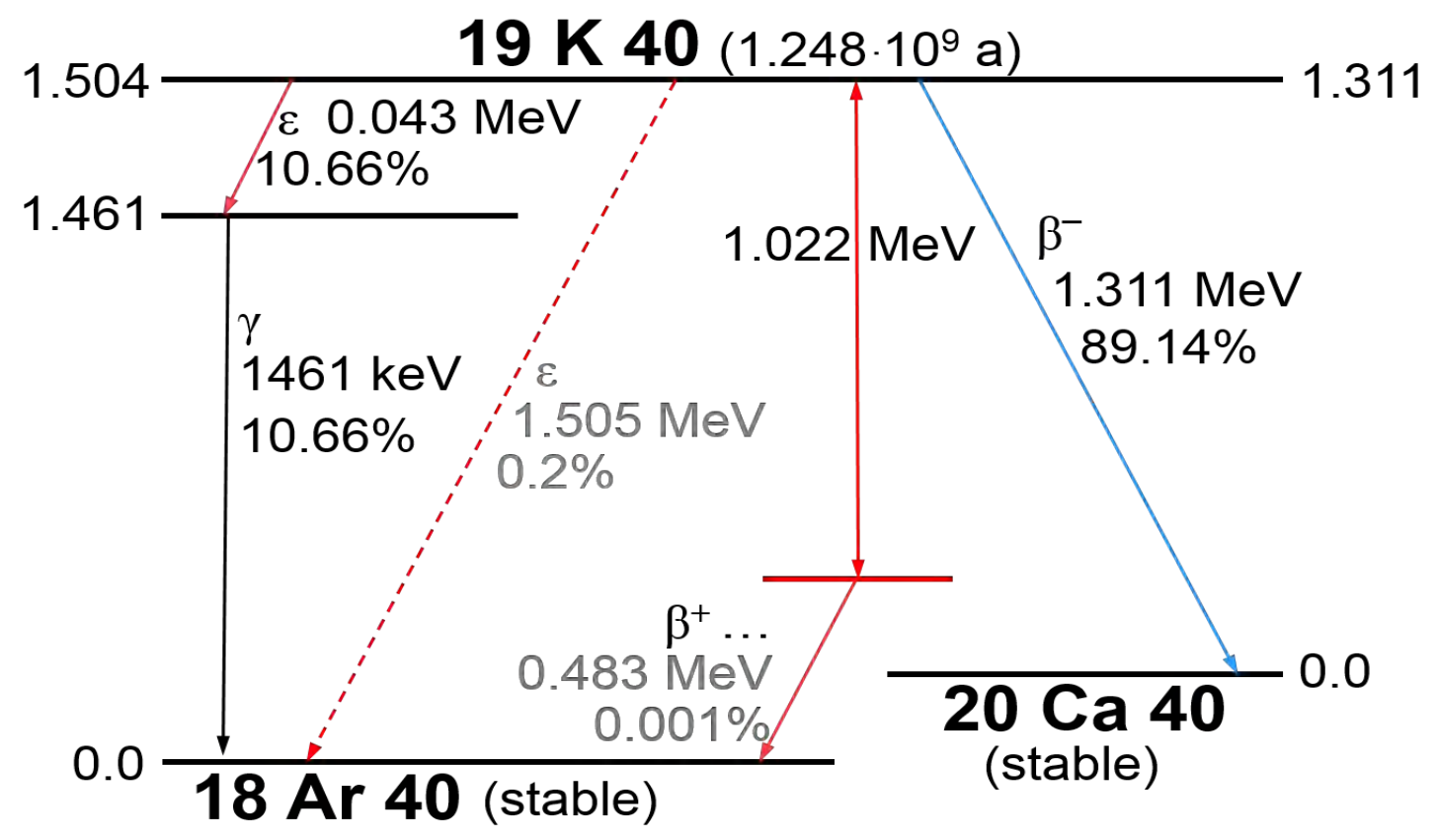

Figure 2.10: Schematic diagram of a ${ }^{40} \mathrm{~K}$ decay 47

Schematic diagrams of ${ }^{40} \mathrm{~K},{ }^{232} \mathrm{Th}$ and ${ }^{238} \mathrm{U}$ can be seen in Figure 2.10, Figure 2.11 and Figure 2.12 respectively. To determine which progeny of these decays contribute to Cherenkov radiation the minimum energy required to produce Cherenkov radiation in acrylic was calculated to be $174.6 \mathrm{keV}$ and $126.1 \mathrm{keV}$ for the PMT glass. Therefore, if a decay has a q-value less then $126.1 \mathrm{keV}$ or is suppressed due to a long half-life or small branching fraction, then it was not considered to contribute. 


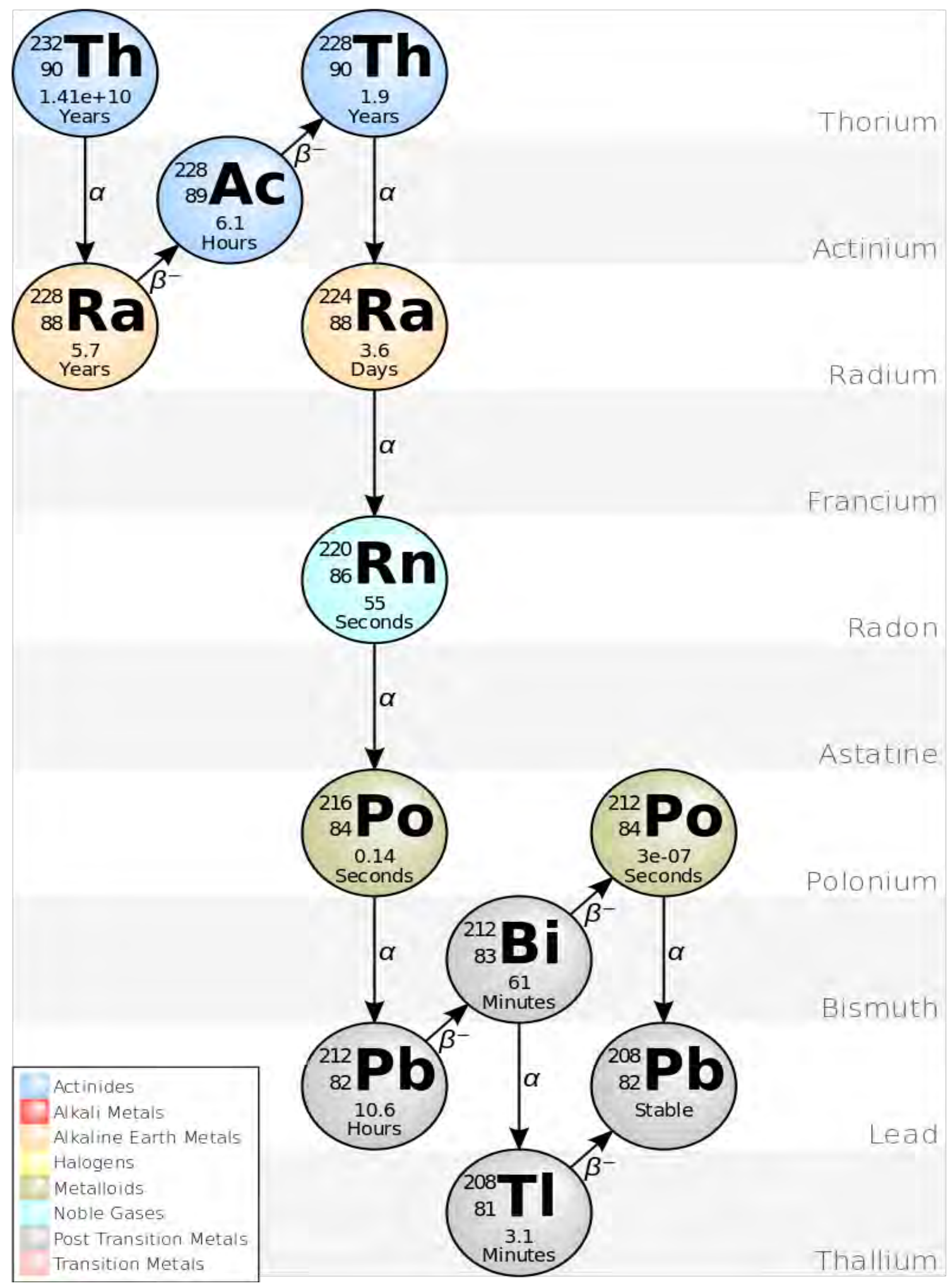

Figure 2.11: Schematic diagram of the ${ }^{232} \mathrm{Th}$ decay chain [48]. 


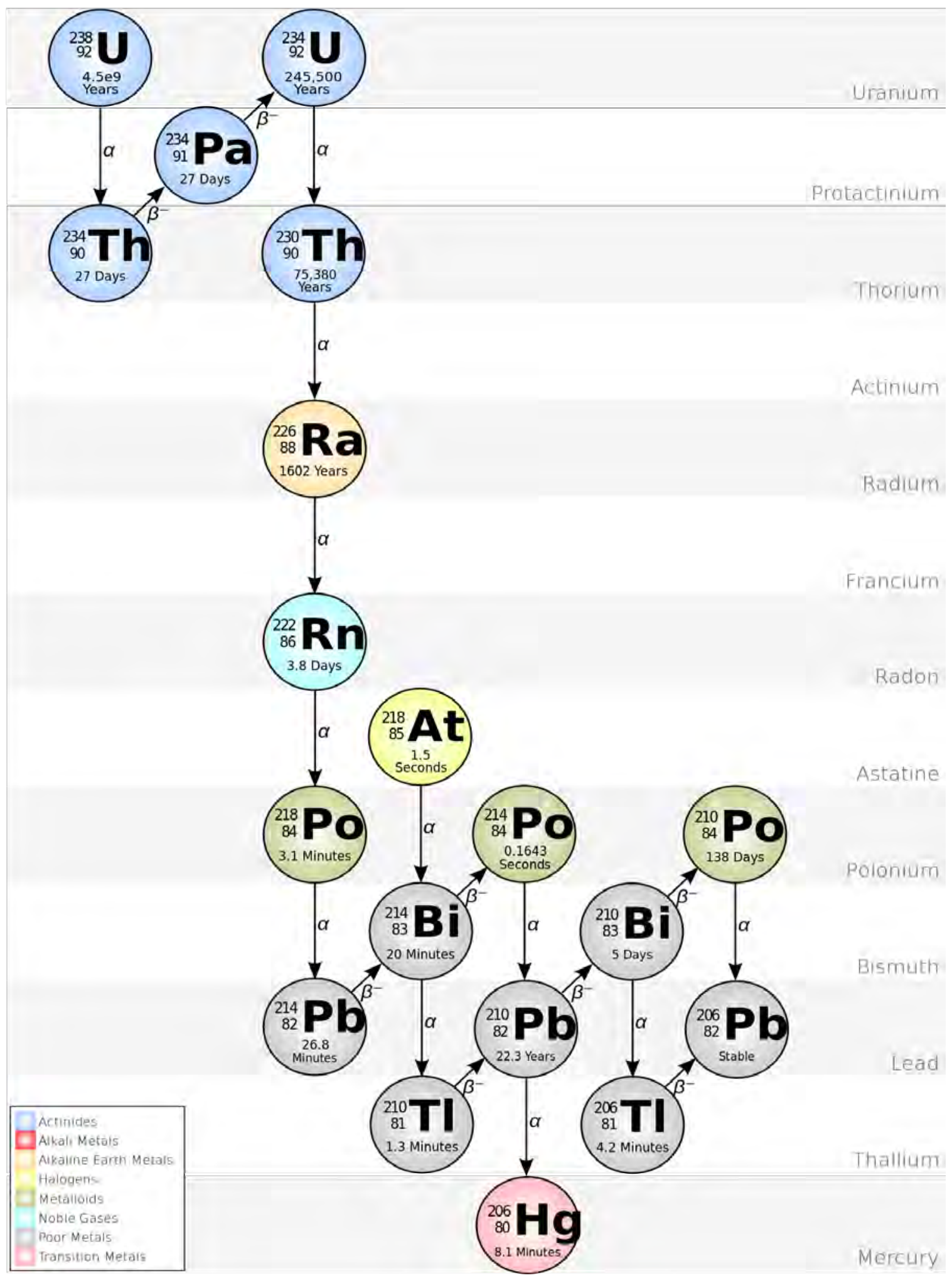

Figure 2.12: Schematic diagram of the ${ }^{238} \mathrm{U}$ decay chain [49]. 


\subsubsection{Alphas}

$\alpha$-decays that are produced in the same uranium and thorium decays seen in Section 2.5.2 have the potential to mimic a WIMP signal in DEAP-3600. Typically an $\alpha$-particle would have an energy in the $\mathrm{MeV}$ range which would be easily distinguished from a possible WIMP signal, as it would produce a very large amount of scintillation light. However, if an $\alpha$-decay occurs in a place such that it does not deposit the entire energy of the decay in to the LAr then it could mimic the expected signal of a WIMP event. The most common way for this to occur in DEAP-3600 would be if an $\alpha$ decay occurred either in the TPB layer or on the inner surface of the AV. These are problematic due to the fact that an $\alpha$-particle would have to travel though a different material (depositing part of its energy into that material) before it would enter the LAr, resulting in a particle with large mass and low energy interacting with the LAr. The easiest way to remove these events is to make a radial cut, removing events that occur at large radii. However, it is still possible for a small fraction of events to travel deeper into the detector and not be removed by a radial cut.

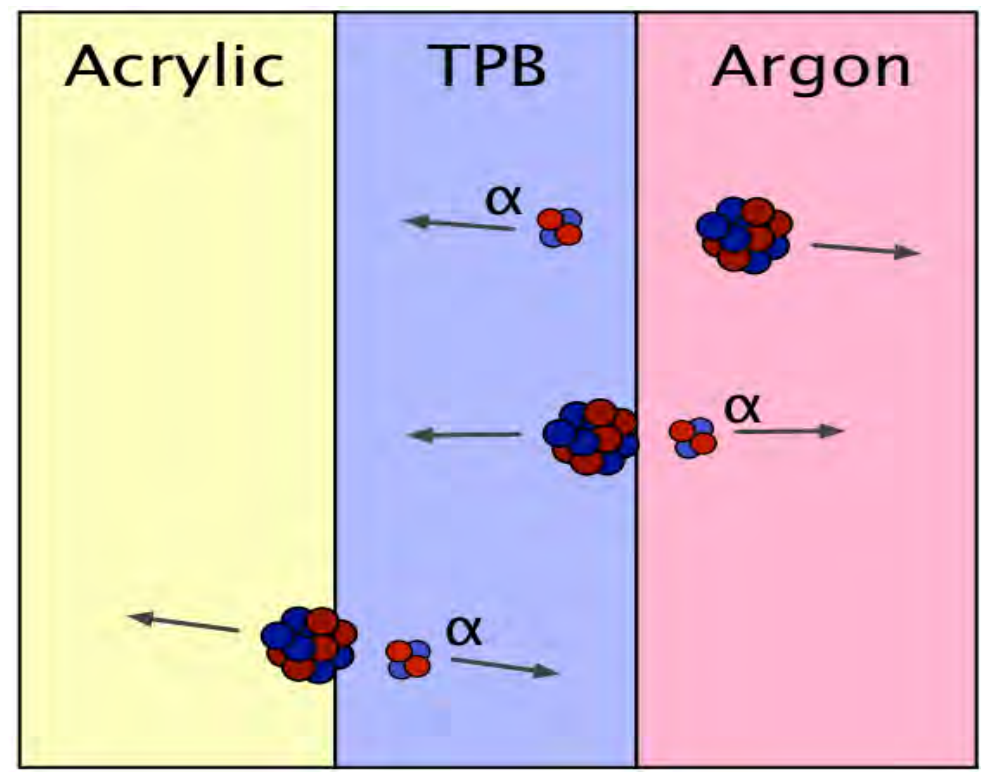

Figure 2.13: Diagram showing the different cases for which an alpha events could mimic a WIMP signal if the event happened on the surface of the AV or in the TPB. 
Another way an $\alpha$-particle can mimic a WIMP signal is if it occurs in neck of the AV. The neck of the AV contains two separate flow guides (FGs) called the inner flow guide (IFG) and outer flow guide (OFG) that are above the LAr fill level. If there is a thin layer of LAr coating the surface of the FGs, scintillation light can be created. A simulation study was done that showed ${ }^{210} \mathrm{Po} \alpha$-decays in the neck cause scintillation light that reaches the AV PMTs [16]. However, since there is no TPB on the surface of the FGs, most of the UV scintillation photons are absorbed by the acrylic, causing a shadowing effect to these events. The shadowing effect can cause these events to mimic WIMP-like signals in the detector.

\subsubsection{Muons}

As mentioned in Section 2.0.1, the muon flux in SNOLAB is $0.27 \mu / \mathrm{m}^{2} / \mathrm{d}$. Although this is a small background, it can still affect the data. In order to mitigate muon event contamination, the detector has been equipped with a muon veto system described in Section 2.1. If a muon enters the detector it will first pass through the water tank producing Cherenkov radiation, then it will pass through the detector causing scintillation light. The Cherenkov radiation in the water tank will be detected by the 48 PMTs that are mounted on the outer surface of the stainless steel shell. If the detector receives a signal from the muon veto PMTs as well as the inner detector at the same time, then the event will be tagged as a muon event and not used in data. $\sim 0.162 \%$ of all trigger that occur have a coincidence with a muon veto trigger.

A possibly more concerning muon event would be one such that it only clips the stainless steel water tank, or very close to the edge of the water tank. These events are concerning due to the fact that they will produce very little to no light (so they could be missed by the muon veto system) as well as they could induce a cosmogenic neutron in the stainless steel. It would then be possible for a neutron to make its way 
into the detector and cause a WIMP like signal. These kind of events would be very hard to remove due to the fact that the veto did not trigger, and there would be no way of knowing the origin of the event. 


\section{Chapter 3}

\section{Random Trigger and Low Threshold Data}

The standard physics trigger used to collect data in DEAP-3600 is not be sufficient for collecting the appropriate data to conduct a low mass WIMP search. The standard physics trigger runs at an energy threshold of $1000 \mathrm{ADC}\left(\sim 75 \mathrm{PE}\right.$ or $\left.\sim 10.14 \mathrm{keV}_{\mathrm{ee}}\right)$; this means that only events that deposit an energy greater then $\sim 10.14 \mathrm{keV}_{\text {ee }}$ in the detector will cause the DAQ to trigger and record their information $\left(\mathrm{keV}_{\mathrm{ee}}\right.$ is the electron recoil equivalent energy discussed in Section 4.1.3). In order to carry out a low mass WIMP search, the goal is to push the sensitivity down in DEAP-3600 to as low of an energy as possible. In this thesis there were two attempts at doing this, using two different trigger settings; the random trigger and a low-energy threshold trigger that was designed for the purposed of this work. This chapter will provide an overview of each trigger, how they work, as well as the advantages and disadvantages to each one. 


\subsection{Random Trigger}

The periodic trigger is a component of the standard physics trigger that is constantly running during all physics runs. The periodic trigger runs as a frequency of $40 \mathrm{~Hz}$, meaning that it forces the detector to trigger 40 times every second. There are two components to the periodic trigger; the pulse pattern generator (PPG) which runs at $1 \mathrm{~Hz}$ and the random trigger which runs at $39 \mathrm{~Hz}$. The PPG is a run type where a voltage signal that mimics an event is injected into different channels of the detector. The purpose for this is to measure and monitor the timing offset between different channels of the detector.

The random trigger is a specialized trigger setting which forces the DAQ to trigger. As a results of the trigger being forced, data with essentially a zero threshold is collected since the DAQ does not have to wait for a signal that is above a given trigger threshold to collect data. The purpose of this is to collect all of the light that is in the detector at the time of the trigger. This is beneficial for looking at low energy events and also very helpful for understanding the trigger threshold since events on both sides of the trigger threshold curve can be analyzed. However, it also introduces a number of issues that must be overcome in order to understand and analyze the data properly.

As seen in Figure 3.1, the primary issue with using the random trigger is recording events with well calibrated trigger times (trigger times that leave enough time after

the pulse for a full $\mathrm{F}_{\text {prompt }}$ integration window). Because all of the information that is in the detector is being collected, only the tail of an event (red curve) or an incomplete event (green curve) are often captured. This is problematic to the analysis because the PSD variable $F_{\text {prompt }}$ relies on there being at least $10 \mu \mathrm{s}$ of recorded time after the main pulse of the signal to be calculated properly (Section 4.1.4). To alleviate this a new trigger time processor was written as part of this work which is discussed in Section 4.2. Along with this, there is another possible issue known as trigger 
shadowing. Trigger shadowing occurs when the timing for a random trigger to occur coincides with a physics trigger that is still active. Given this scenario, the physics trigger is recorded and the random trigger has to wait until the next time in the $39 \mathrm{~Hz}$ cycle to trigger. In cases where trigger shadowing, is present random triggers can be missed, thus affecting the observed event spectra for this trigger type.

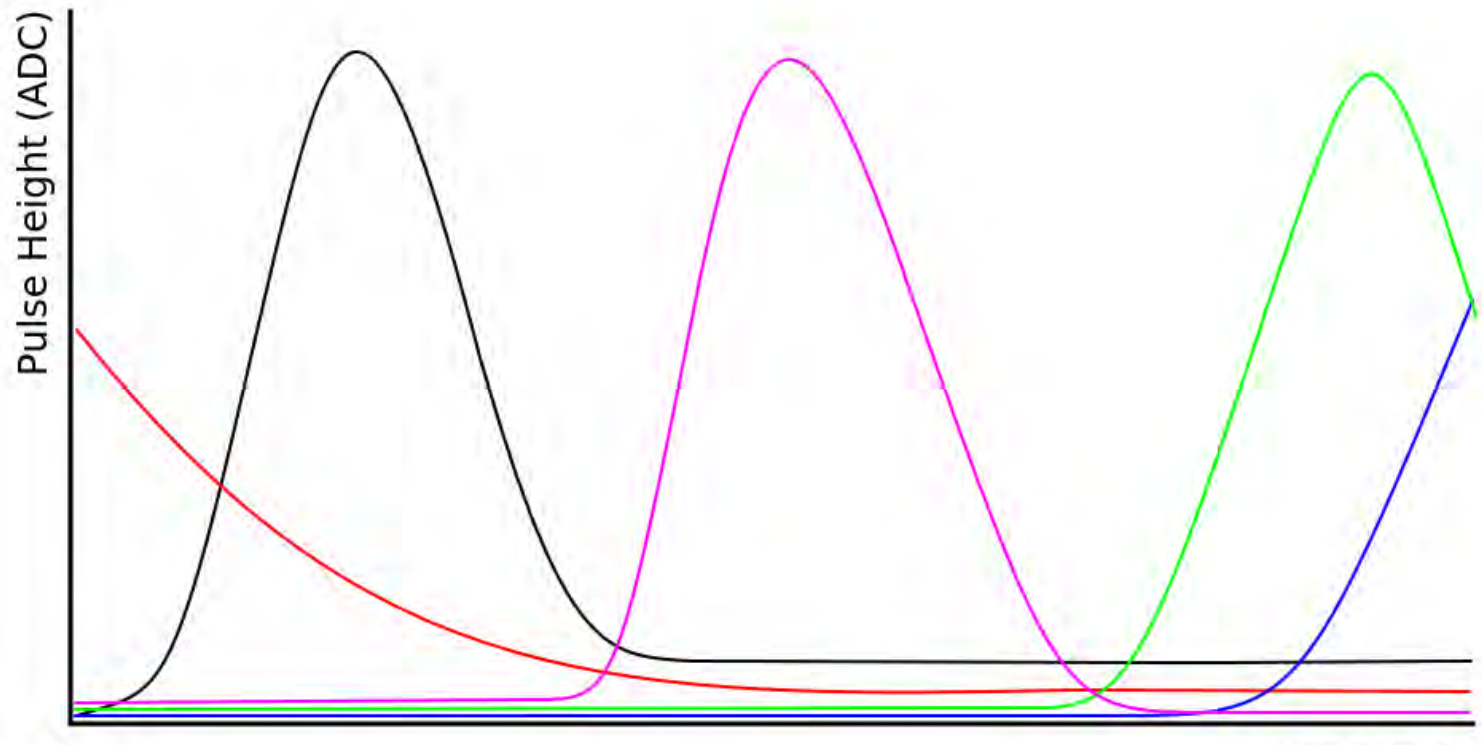

Time (ns)

Figure 3.1: Diagram of waveforms that can be found in random trigger data. The black curve shows a waveform like one that can be found in standard physics data, the red curve shows the case of capturing only the tail of an event, the magenta curve is if a full event is captured outside the calibrated trigger time window, the green curve is if the detector is captures an event late in the time window missing the tail, and the blue curve is if the detector captures a small part of the start of an event very late in the time window.

\subsection{Low-Energy Threshold Data}

The low-energy threshold trigger is a hardware trigger that was designed and developed specifically this work. The motivation for creating this trigger is to allow for the collection of data that can probe lower energy events, but does not become washed out by noise. To achieve this, the energy threshold required for the DAQ to 
record a trigger had to be lowered to a value that is lower then the current standard physics trigger energy threshold of $\sim 1000$ ADC. An important thing to note is that this is a dedicated physics trigger with a lower threshold and does not run in unison with the standard detector physics trigger that is used for other physics analyses. Because the low-energy threshold trigger is an independent configuration, there will be no observed trigger shadowing that is seen in random trigger data. Given that there is energy threshold requirement (albeit low) for the DAQ to trigger, the events collected with the low threshold trigger have well calibrated events times.

\subsubsection{Determining the Low-Energy Threshold Trigger Set- tings}

In order to determine an appropriate threshold for the low-energy threshold trigger a series of short runs were taken over a range of trigger energy thresholds from 60 ADC to 1000 ADC. The data from these runs can be seen in Figure 3.2. Basic pile-up remove cuts (discussed in Section 4.1) have been applied to the data in order to get an idea of how much useful data (data without pile-up or noise) is taken by each trigger threshold. It can be seen in Figure 3.2 that as the trigger threshold is reduced the sensitivity to lower energy events increases. This can be seen as the 1000 ADC threshold spectrum goes down to $\sim 15$ qPE, where as the 100 ADC threshold spectrum goes down to $\sim 1 \mathrm{qPE}$. Indicating that lowering the trigger threshold will record events with a lower energy as expected. The other thing to consider is the rate of events which are recorded by each threshold in order to collect that largest amount of data possible. Above $100 \mathrm{qPE}$ all of the spectra match because region is dominated the ${ }^{39} \mathrm{Ar}$, so all the triggers will record the same information. The focus is on low energy events however, so when determining the highest rate of events, only events that are below $100 \mathrm{qPE}$ are considered. The highest low energy peak in Figure 3.2 occurs at a threshold of 150 ADC. Showing that running at 150 ADC provides the 
highest rate of useful data, while still increasing the sensitivity to low energy events. For these reasons $150 \mathrm{ADC}(\sim 11.25 \mathrm{PE}$ or $\sim 1.52 \mathrm{keV})$ was chosen as the operating energy threshold for the low threshold trigger.

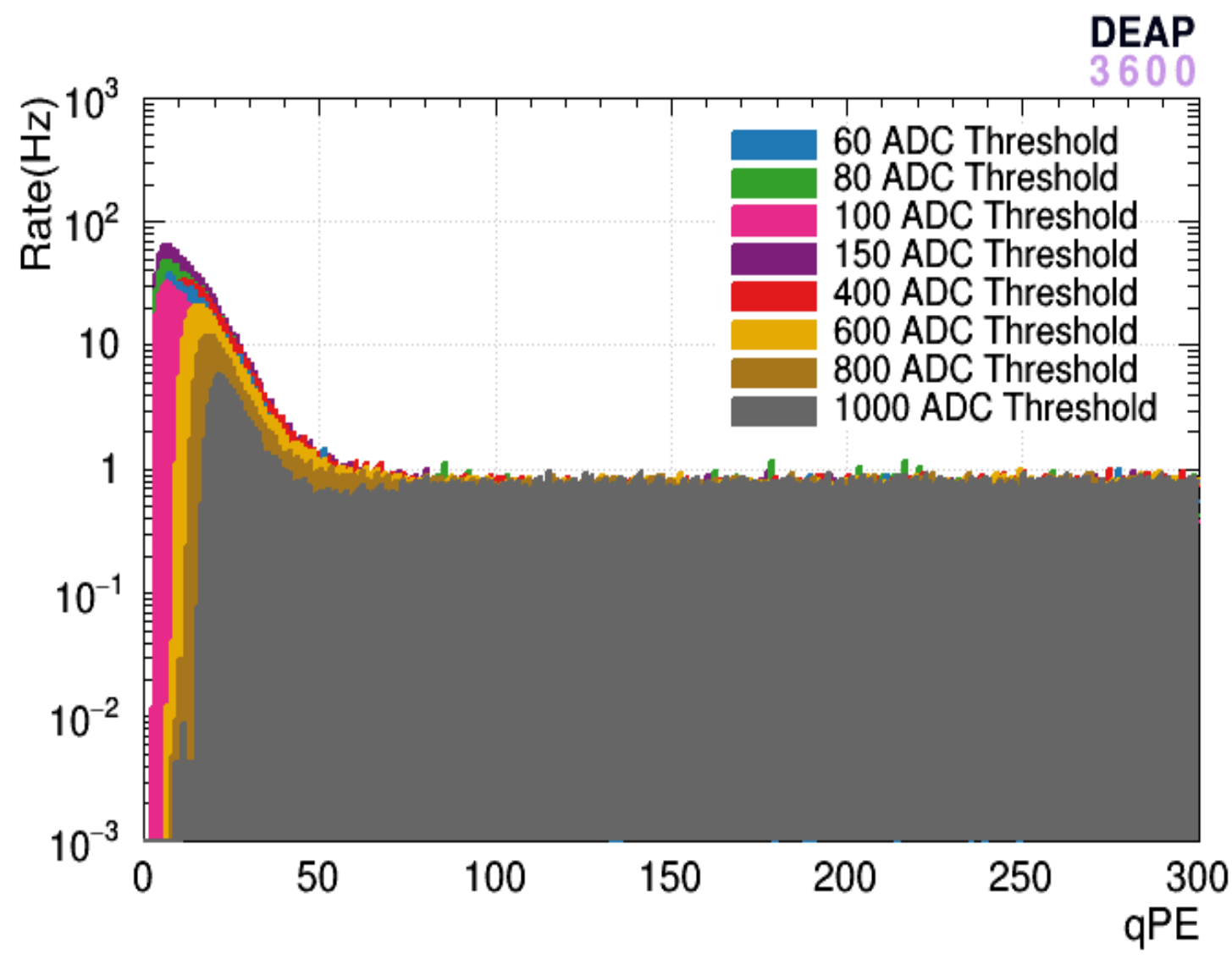

Figure 3.2: qPE spectra taken with various hardware trigger thresholds. It can be seen that as the energy threshold of the trigger is lowered, the sensitivity to low energy events increases, as well as the rate of low energy events increase until $100 \mathrm{ADC}$ when that amount amount of pile-up events out weighs the amount of useful data.

\subsubsection{Low-Energy Threshold Data Set}

The low threshold data set was taken as a dedicated run request that was completed in December 2018. The data set has an exposure time of 3.02 days and was collected over one and a half weeks. It was decided that the collection period would be on different days over one and a half weeks to ensure that enough data would be 
collected even if something had gone wrong with one of the runs such that the data could not be used. The mass of LAr in the detector is $(3279 \pm 97) \mathrm{kg}$ (Appendix A), this gives a total exposure of $9.903 \times 10^{3} \mathrm{~kg}$ - days before any cuts are applied.

The final trigger setting for this data set is nearly identical to the standard physics trigger with the only modifications being that the energy threshold is reduced from $1000 \mathrm{ADC}$ to $150 \mathrm{ADC}$ and only very low energy and low energy events (Regions A, $\mathrm{B}$, and $\mathrm{C}$ in Figure 2.5) are sent to the digitizers for processing since these are the focus of this work. 


\section{Chapter 4}

\section{Low-Energy Threshold Analysis}

This chapter will discuss the analysis that was performed for this work. It will start with a description of the variables that are used by the DEAP-3600 collaboration and discuss some of the issues that can arise when looking at low-energy threshold data. Then a description of a the new trigger time processor, called the low threshold trigger time processor, that was created for this work. After this a discussion about low energy background events and how to remove them, including a description the Cherenkov model in DEAP-3600 that was constructed as part of this work. This chapter will conclude with a description of the final signal extraction algorithm that was used.

\subsection{Analysis Variables}

The majority of the information that is collected by the detector is output in the form of waveforms that are produced by all the PMTs. Each set of waveforms that are produced by a single event contain a lot of information that can be extracted; the DEAP-3600 collaboration has developed a large number of variables based on waveform information that can be used to characterize events.

The following variables can be used to select events that are appropriate for a 
low mass WIMP analysis. These variables were selected with the purpose of focusing mainly on simple variables. Simple in this case means variables that use very low level information such as charge and counting statistics. DEAP-3600 has other analyses that use very high level statistics utilizing fits, charge corrections, multivariate analysis, and machine learning which would not be suitable for a low energy analysis. This is because when looking at low energy events, the energy deposited becomes more discrete and there is less information that can be used for such an analysis. For example in Figure 2.8, as energies approach lower values, PSD begins to break down as electronic and nuclear recoils begin to overlap below $70 \mathrm{qPE}$. This means that PSD would not be suitable for a low energy analysis, which is why a background subtraction analysis was used in this work instead (discussed in Section 5.3).

\subsection{1 calcut}

\begin{tabular}{c|c|c}
\hline Bit & Hex Code & Description \\
\hline \hline 0 & $0 \times 1$ & If a V1720 pulse had a bad baseline \\
1 & $0 \times 2$ & If a V1720 pulse reached 0 ADC (saturation) \\
2 & $0 \times 4$ & Failed to find a "good" calibrated trigger time \\
3 & $0 \times 8$ & If this is a PPG (not used for physics analysis) \\
4 & $0 \times 10$ & If this is soon after a PPG event \\
5 & $0 \times 20$ & If a spare V1720 has a pulse (due to PPG or noise) \\
6 & $0 \times 40$ & DAQ was running busy and suppressing readout of digitizers \\
7 & $0 \times 80$ & If the trigger/digitizers are out of sync \\
8 & $0 \times 100$ & If event timestamps are appearing out-of-order \\
9 & $0 \times 200$ & There are no digitizers in the event (due to pre-scaling) \\
10 & $0 \times 400$ & If event came from a non-physics trigger source \\
11 & $0 \times 800$ & If SQT info is used for a non-SPE-like pulse \\
12 & 0x1000 & If SQT info is used for a pulse with a truncated charge integral \\
13 & 0x2000 & If SQT info is used for a pulse > 1000 pC \\
16 & 0x10000 & If LowThreshTrigTime processor fails \\
\hline \hline & 0x31f8 & Removes any instances of bits 3, 4, 5, 6, 7, 8, 12 and 13 \\
\hline
\end{tabular}

Table 4.1: List of all calcut bitmasks used in event selection cuts. Smart QT (SQT) is an algorithm that is applied in the V1720 digitizers to encode waveforms. 
calcut is a variable that is set as a flag in the data with the main purpose of removing events not suitable for physics analysis. These flags can indicate scenarios such as a PPG event, the DAQ being not calibrated properly, or the DAQ failing. A summary of the bitmask flags available in the calcut variable and the corresponding definitions of what each one removes can be seen in Table 4.1. The current cut value for calcut is 0x31f8 (in hex) which removes all events that correspond to bits $3,4,5$, $6,7,8,12$, and 13 .

\subsection{2 dtmTrigSrc}

dtmTrigSrc is a variable that describes what trigger was used to observe a given event. Like calcut, dtmTrigSrc implements a bitmask flag in the data to indicate the source of the trigger. A summary of the dtmTrigSrc bitmasks and their descriptions are given in Table 4.2. The current dtmTrigSrc cut is 0x82 (in hex) which removes instances of bits 2 and 8 .

\begin{tabular}{c|c|c}
\hline Bit & Hex Code & Description \\
\hline \hline 2 & $0 \times 2$ & Internal periodic trigger \\
8 & $0 \times 80$ & External calibration trigger \\
11 & $0 \times 400$ & Low energy, low $F_{\text {prompt trigger }}$ \\
12 & $0 \times 800$ & Low energy, high $F_{\text {prompt }}$ trigger \\
13 & $0 \times 1000$ & Medium energy, low $F_{\text {prompt trigger }}$ \\
14 & $0 \times 2000$ & Medium energy, high $\mathrm{F}_{\text {prompt }}$ trigger \\
15 & $0 \times 4000$ & High energy trigger \\
\hline \hline & $0 \times 82$ & Removes any instances of bits 2 and 8 \\
\hline
\end{tabular}

Table 4.2: List of dtmTrigSrc bitmasks used in event selection cuts.

The internal periodic trigger is the same as the periodic trigger which is described in Section 3.1. The external calibration trigger corresponds to events that are triggered by the muon veto system. The medium energy, low $\mathrm{F}_{\text {prompt }}$ (bit 13) events are the events which undergo ${ }^{39} \mathrm{Ar}$ pre-scaling by a factor 1:100 which is described in Section 2.5.1. Based on the description of the low threshold trigger in Section 3.2.1 
and the information in Table 4.2 it can be seen that the bits 13, 14, and 15 are set to $0 \mathrm{x} 0$ (in hex) in the low threshold trigger settings. This means that no information above $\sim 450 \mathrm{PE}$ will be sent to the digitizers. The focus of this work is on events in the low energy range that correspond to bits 11 and 12 .

\subsection{3 qPE}

The qPE variable is an estimate of the total measured number of photoelectrons (PE) that are produced by an event. This can then be related to energy though the understanding of the light yield of the detector. The light yield of the detector is $7.3 \mathrm{PE} / \mathrm{keV}_{\mathrm{ee}}$; through this relationship the electron recoil equivalent (and nuclear recoil equivalent, $\mathrm{keV}_{\mathrm{nr}}$, using the nuclear quenching factor) energy can be determined.

DEAP-3600 performs two to three PMT calibration runs daily using a system called the acrylic and aluminum reflector fibre (AARF) system. The AARF system consists of acrylic fibers with their ends located near 20 PMTs located uniformly around the AV. At the end of each fiber is an acrylic stud that is coated in reflective aluminum. $435 \mathrm{~nm}$ light is injected through the fibres and reflects off of the stud toward a given PMT; part of the light is observed by the PMT and the other portion of light reflects off the PMT glass and redirects into the detector. From the AARF calibration runs the average charge read out by the PMTs due to a single photoelectron (SPE) can be determined. Using the calibration from AARF runs the qPE value of each pulse can be determined from signals collected in physics data. This returns a spectrum as shown in Figure 4.1. After applying the cut selection for the low threshold trigger, the spectrum can be seen in Figure 4.2 . 


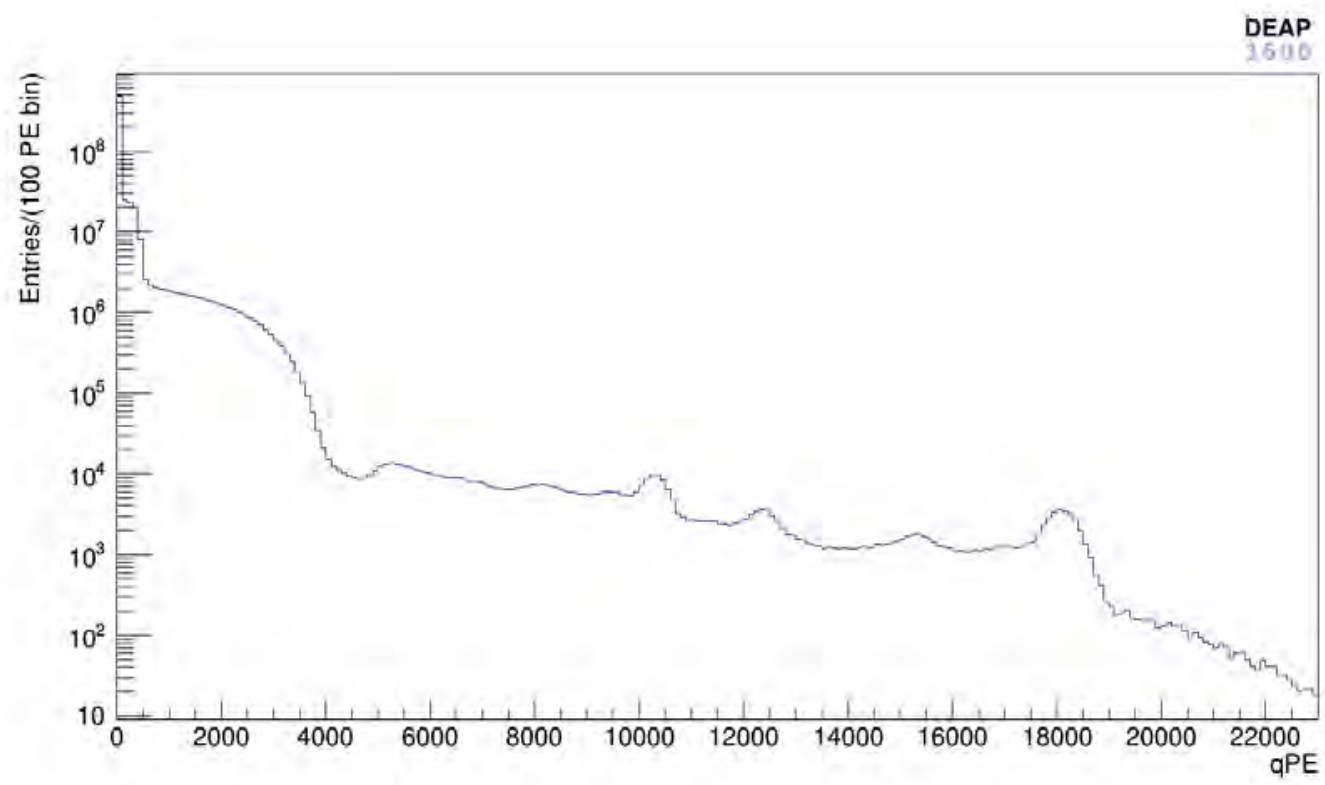

Figure 4.1: The qPE spectra that is measured by DEAP-3600. The region from $\sim$ $450 \mathrm{qPE}$ to $4000 \mathrm{qPE}$ is the pre-scaled ${ }^{39} \mathrm{Ar}$ region. The peak at $\sim 10000 \mathrm{qPE}$ is due to $1461 \mathrm{keV}$ gammas from ${ }^{40} \mathrm{~K}$ decays, the peaks at $\sim 12000 \mathrm{qPE}$ and $\sim 15500 \mathrm{qPE}$ are due to $1765 \mathrm{keV}$ and $2204 \mathrm{keV}$ gammas from ${ }^{214} \mathrm{Bi}$ decays, and the peak at $\sim 18000 \mathrm{qPE}$ is due to $2614 \mathrm{keV}$ gammas from ${ }^{208} \mathrm{Tl}$ decays.

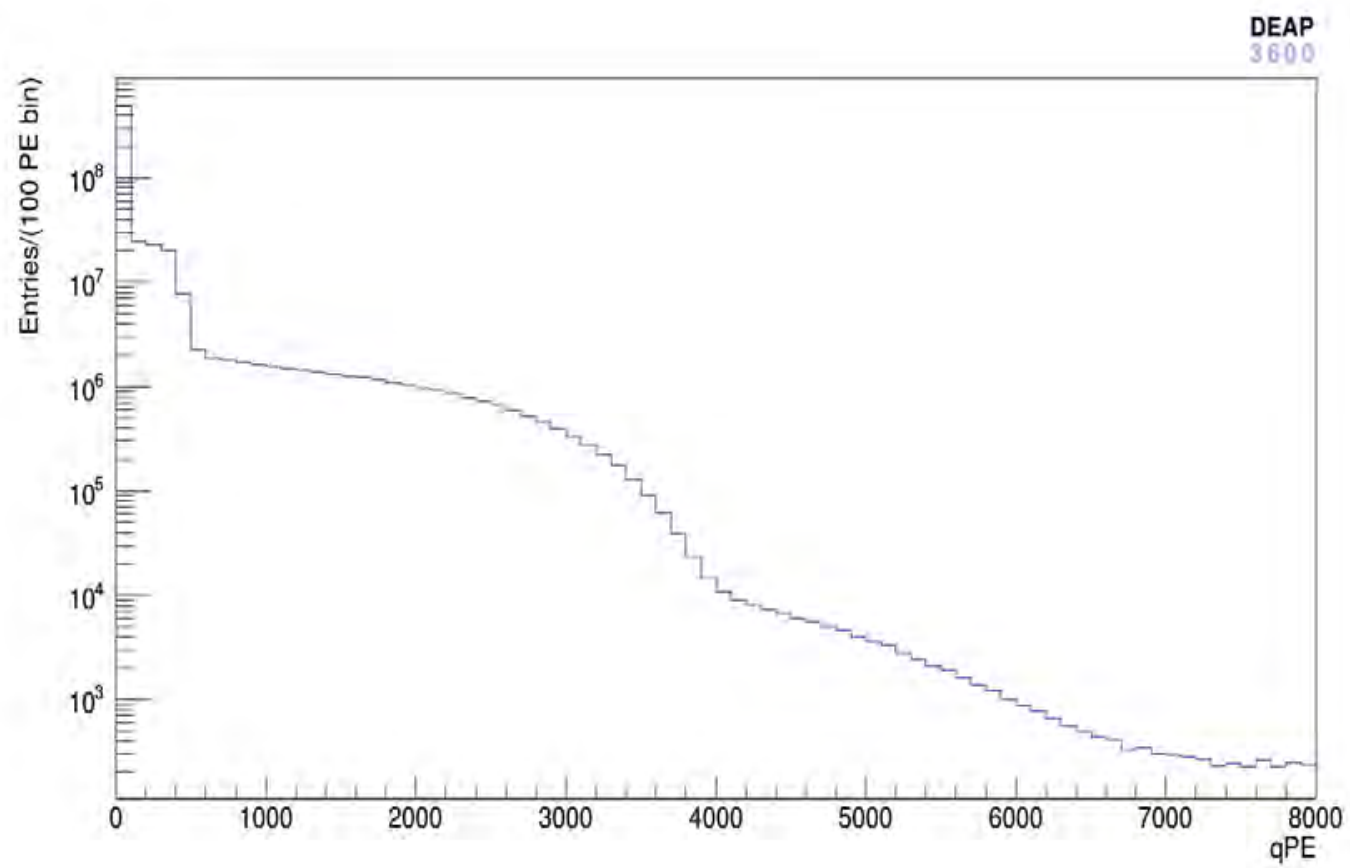

Figure 4.2: $\mathrm{qPE}$ spectra measured by the low threshold trigger. The region from $\sim 450 \mathrm{qPE}$ to $4000 \mathrm{qPE}$ is the pre-scaled ${ }^{39} \mathrm{Ar}$ region. The region from $\sim$ $4000 \mathrm{qPE}$ to $7000 \mathrm{qPE}$ is ${ }^{39} \mathrm{Ar}$ pile-up. 


\subsection{4 $\quad \mathrm{F}_{\text {prompt }}$ and $\mathrm{F}_{\operatorname{maxPE}}$}

$\mathrm{F}_{\text {prompt }}$ is the PSD variable used throughout the DEAP-3600 analysis. Demonstrated in Section 2.4, $\mathrm{F}_{\text {prompt }}$ is a powerful tool that can be used to discriminate between electronic recoil and nuclear recoil events (as well as Cherenkov radiation events). $\mathrm{F}_{\text {prompt }}$ is defined as the fraction of charge collected from all the inner detector PMTs in the first $150 \mathrm{~ns}$ of a pulse to the charge collected over $10 \mu \mathrm{s}$ of a pulse. Figure 4.3 shows an example of a waveform (collected by DEAP-3600) with the $\mathrm{F}_{\text {prompt }}$ integration windows highlighted. Red is the $150 \mathrm{~ns}$ (prompt) window, blue is the $10 \mu$ s (wide or late) window and purple is the overlap of the two windows. The functional form of $\mathrm{F}_{\text {prompt }}$ can be seen in Equation 4.1 .

$$
F_{\text {prompt }}=\frac{\int_{t_{0}}^{t_{150 n s}} Q(t) d t}{\int_{t_{0}}^{t_{10 \mu s}} Q(t) d t} .
$$

The variable $\mathrm{F}_{\operatorname{maxPE}}$ is defined as the maximum fraction of total $\mathrm{qPE}$ that is counted in a single PMT. This is similar to $\mathrm{F}_{\text {prompt }}$ except instead of using prompt light versus total light, the variable uses the fraction of light seen by a single PMT (which sees the most light) versus the fraction of light seen by all the PMTs. Due to the fact that these variables are calculated in a similar way, they become more correlated at lower energies. This is because a low energy event is only observed by a small number of PMTs and most of the light travels to a single PMT. Due to the low energy nature of the event, there would also be very little late light resulting in similar $\mathrm{F}_{\operatorname{maxPE}}$ and $\mathrm{F}_{\text {prompt }}$ values.

$\mathrm{F}_{\operatorname{maxPE}}$ is useful for removing events that happen near the inner surface of $\mathrm{AV}$. $\mathrm{F}_{\operatorname{maxPE}}$ is useful for removing events near the surface or potentially Cherenkov radiation that occurs in the LGs or PMT glass because if an event happens close to a LG most of the light will travel to a handful of PMTs with a large fraction going to a single PMT. In this case, the event would produce a high $\mathrm{F}_{\operatorname{maxPE}}$ value compared to 
an event the happens in the inner detector that would be observed by many PMTs. However, when looking at low energy events, making cuts with $\mathrm{F}_{\operatorname{maxPE}}$ must be done with caution due to the fact that there could be a genuine low energy event that occurs near the surface that could also be removed.

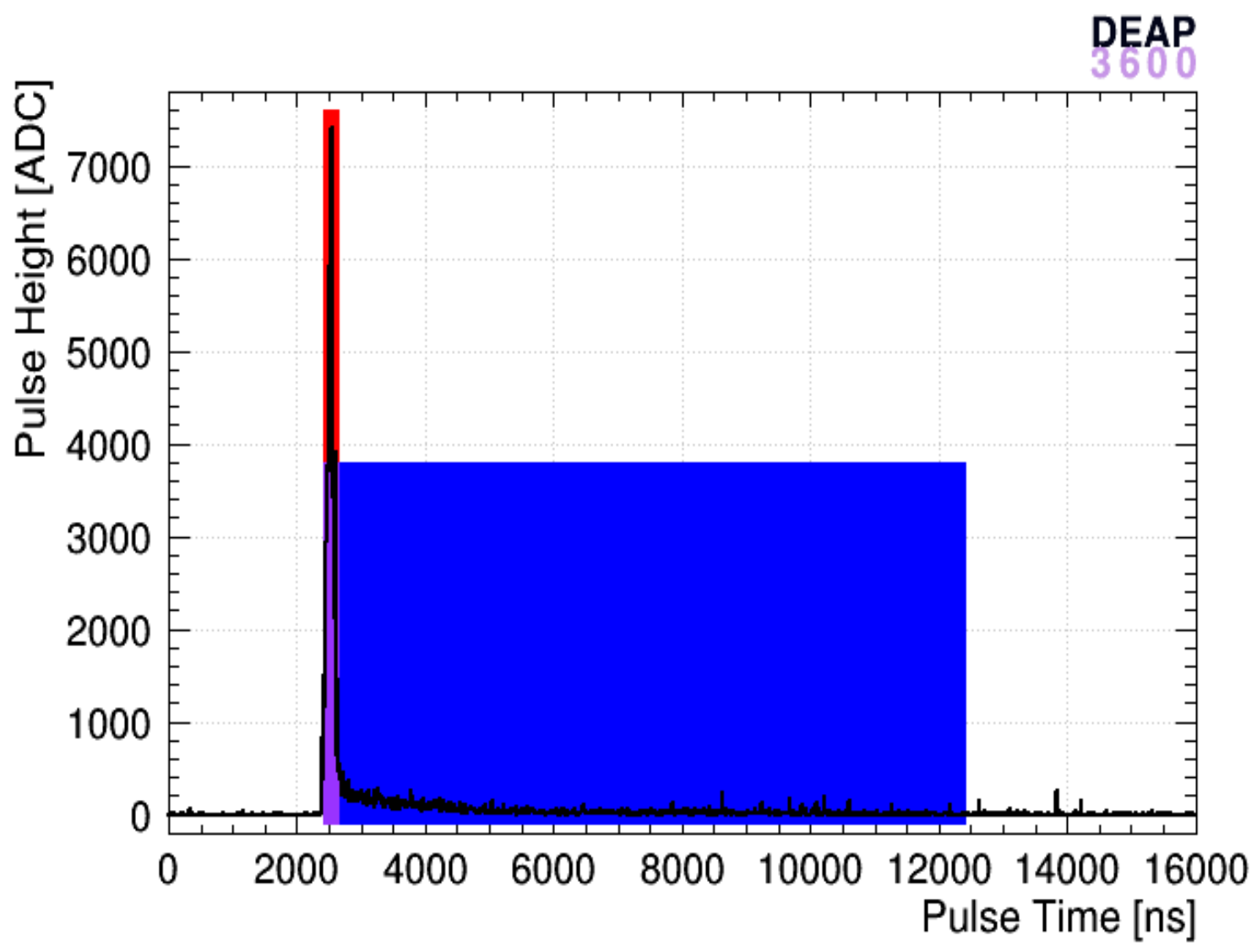

Figure 4.3: A standard waveform from DEAP-3600 data with the $\mathrm{F}_{\text {prompt }}$ integration windows overlaid. The prompt window can be seen in red, the wide window can be see in blue and where the two windows overlap can be seen in purple.

\subsubsection{Pile-Up Removal Variables}

A pile-up event in DEAP-3600 is defined as the scenario when more then one event occurs with in the same trigger window. Since the event rate in DEAP-3600 is very high, this is a common occurrence. The pile-up rate of ${ }^{39} \mathrm{Ar}^{39} \mathrm{Ar}$ coincident events can be estimated to be $131 \mathrm{~Hz}$ for this data set. This is based off ${ }^{39} \mathrm{Ar}$ trigger 
rate of $3115 \mathrm{~Hz}$ (LAr mass multiplied by the ${ }^{39}$ Ar specific actvity disccused in Section 2.5.1. The collaboration has developed several variables such as subeventN, numEarlyPulses, and deltaT (all defined below) that can be used to remove such events when analyzing the data.

\section{subeventN - Number of pulses in a given waveform, used for event selection cuts.}

The subeventN variable scans through a given waveform and identifies if it contains any subevent signals that occur after the initial event that triggered the DAQ. This is used to separate single recoil events from waveforms that contain at least one subevent in the same $\mathrm{F}_{\text {prompt }}$ integration window. Only single recoil events are used for analysis because if there are any secondary events in the wide window of the $\mathrm{F}_{\text {prompt }}$ integration it would skew that calculation to low $\mathrm{F}_{\text {prompt }}$ values. An example of multiple subevents in a waveform can be seen in Figure 4.4 .

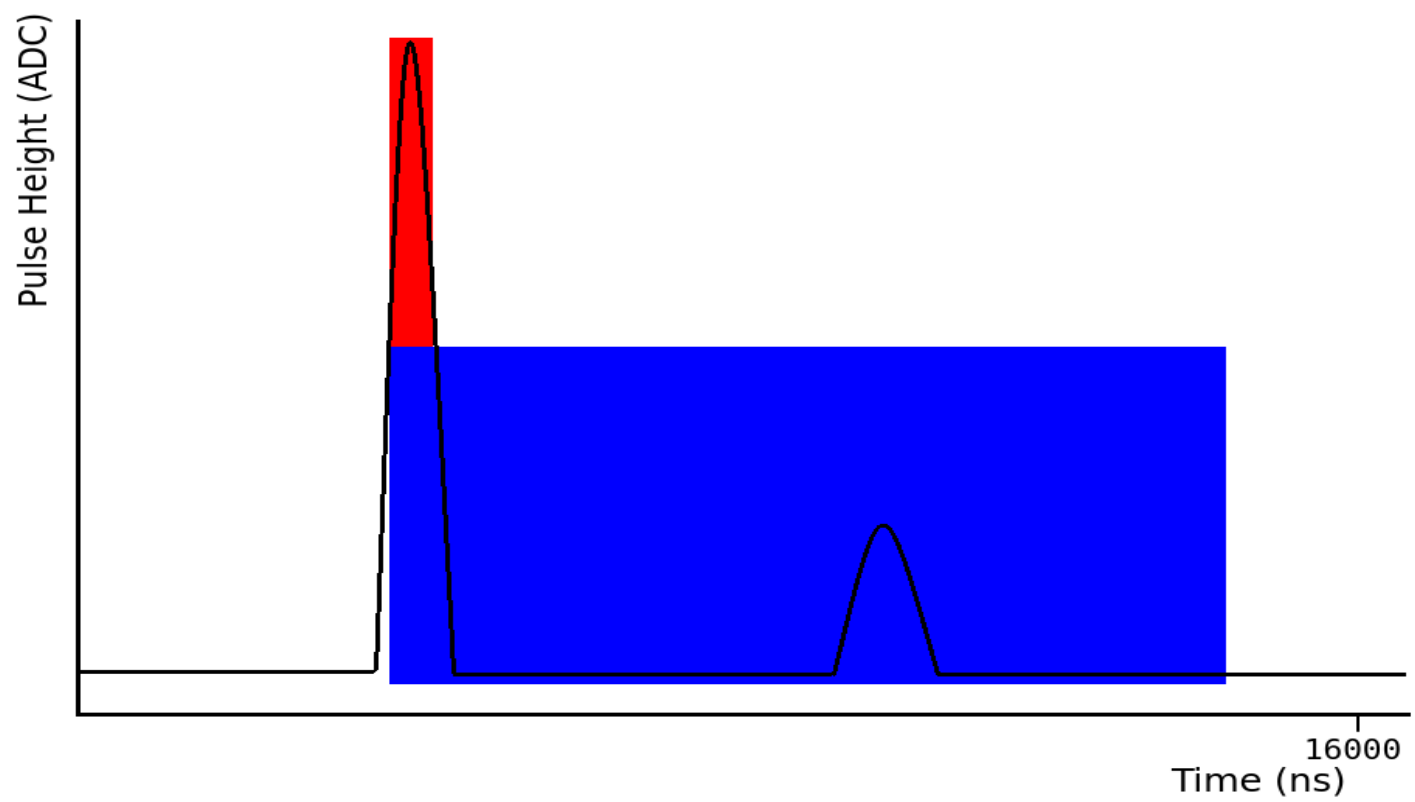

Figure 4.4: Example a waveform the would have subeventN=2. Main pulse can be seen in the prompt (red) window and the secondary pulse can be seen in the wide (blue) window. 


\section{numEarlyPulses - Number of pulses that are found in the pre- trigger window of a waveform.}

The numEarlyPulses variable scans through a given waveform to identify how many PMT pulses occur in the pre-trigger window before the initial event that caused the DAQ to trigger. This provides information such as the tail of the previous event is leaking into the trigger window of the current event. Again, only single recoil events are used in data, so if there are mutiple triggers in quick succession then it is possible for the tail of the first event to leak into the next event. This would add charge to the second event that can cause the event to be miss classified. Events that contain greater then three pulse peaks in the pre-trigger window are removed.

\section{deltaT - Time between two consecutive events; used for event selection cuts.}

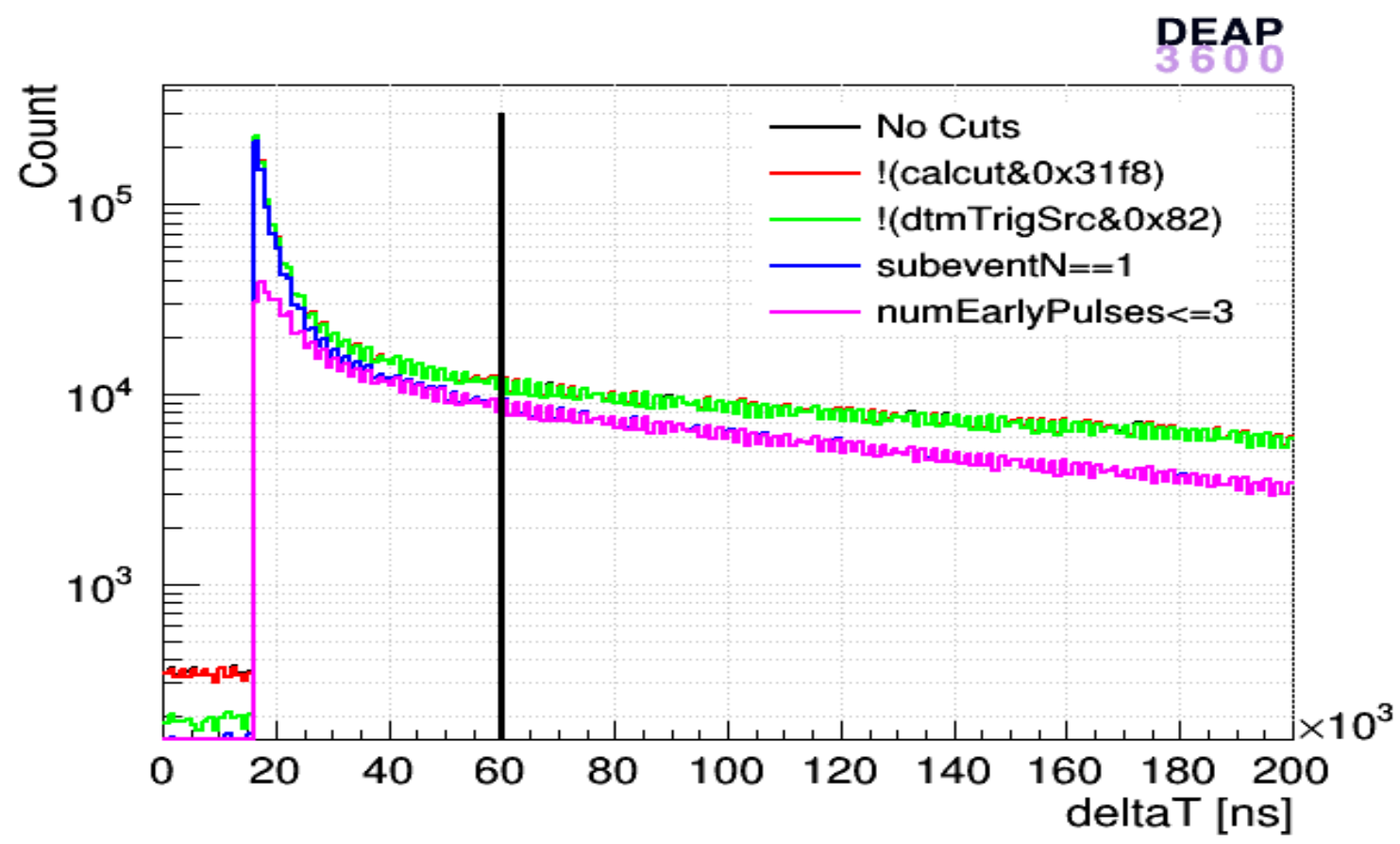

Figure 4.5: deltaT distrbution for low threshold data. The black line represent that deltaT cut at $60000 \mathrm{~ns}$, meaning that all events that have a deltat greater then 60000 ns are kept. 
The deltaT variable is the measured difference in timestamps at the beginning of two event time windows that occur as successive detector triggers. This is used to remove events that occur very close in time to one another (much like numEarlyPulses). Events that occur close in time can be problematic because there may be light from the tail of the first event that leaks into the integration window of the subsequent event. The deltaT distribution of low threshold data is shown in Figure 4.5. A deltaT cut is made at $60000 \mathrm{~ns}$ because the goal is to remove the deltaT values for when the distribution does not behave exponentially (exponential appears linear in Figure 4.5 because of log scale). The deviation from an exponential form below $60000 \mathrm{~ns}$ is caused by pile-up from events occurring in quick succession after one another.

\subsection{6 eventTime - Time of the main pulse in a waveform; used for event selections cuts.}

The eventTime is a measure of where the initial pulse peak of the event is located in time. The current eventTime cut selects events that have eventTimes that are between $2300 \mathrm{~ns}$ and $2650 \mathrm{~ns}$. This is set with the knowledge that a typical well calibrated event will occur around $2500 \mathrm{~ns}$. The $2300 \mathrm{~ns}$ cut is a pre-trigger pile-up cut that will remove any charge that is produced by the tail of a preceding event that may have been missed by other cuts (numEarlyPulses and deltaT). The $2650 \mathrm{~ns}$ cut is a post-trigger pile-up cut that is strongly correlated with subeventN, though there are some instances where this is not true due to the subeventN cut not being $100 \%$ efficient, therefore this cut is in place. 


\subsubsection{Position Reconstruction Variables}

Position reconstruction variables are used to determine where in the detector an event occurred. This can be useful for removing events that happen close to the surface of the AV or outside the AV (Cherenkov) in the LGs or PMT glass, which is important in cleaning the data to select recoil-like events only. Position reconstruction variables are also useful for the removal of retriggers. Retriggers produce an anisotropic distribution of charge in the detector which causes these events to reconstruct near the surface of the AV. Because these events tend to reconstruct near the radial position cuts can be applied to remove them.

\section{centroidR - Reconstructed radius of the central location of charge.}

The centroidR variable is a measure of the geometrical center of the charge of an event. It is calculated by determining the position of a PMT that observes charge and multiplies the position of the PMT by the charge that is observed. The sum of the weighted positions of all the PMTs that observe charge is then calculated and divided by the total charge of the event; leaving a vector to the geometric center of charge of the event. The functional form of of centroidR is given by Equation 4.2, centroidR is useful for low energy analysis because it only requires the charge observed by the PMTs and the PMT locations (which is fixed) to be calculated.

$$
\text { CentroidR }=\frac{1}{q_{\text {tot }}^{2}} \Sigma_{i}\left(\vec{r}_{i} \times q_{i}^{2}\right) .
$$




\section{mblikelihoodR - Reconstructed radius of an event; used for event selection cuts.}

mblikelihoodR is a similar variable to centroidR but uses a likelihood fit to determine the position rather then averaging the position. The processor begins by finding the charge observed by each PMT, like centroidR, but has six options for charge variables; charge integral (most simple), single photoelectron counting (nSC), single photoelectron counting (nSCBayesian), $400 \mathrm{~ns}$ integration window, charge integral with after pulsing removal, or charge integral with qPE. For this work the first option, charge integral, was used. After the charges are calculated for each PMT, the charges, PMT efficiencies, and PMT positions are passed to the fitter, which returns a radial mblikelihood position for the event. miblikelihoodR is useful for making radial cuts in the detector to remove surface events, Cherenkov radiation, and retriggers.

\subsection{Low Threshold Data Processor}

The low threshold trigger processor is a data processor that was created to define calibrated trigger times for low energy events. When looking at low energy events it is possible that the DAQ may trigger on a low energy event, then at some later time in the integration window there is a larger pulse. This is problematic because the standard trigger time processor works by scanning through a waveform searching for the largest charge peak and calls this peak the trigger time. In the scenario that was described above, this would not be an efficient method for determining the trigger time for low energy events because many events can be missed due to having small initial pulses and larger late pulses.

The low threshold trigger time processor works in a similar way to the standard trigger time processor with some modifications. The processor takes in data in the raw or cal formats; if the user desires, $\mathrm{F}_{\text {prompt }}$ and $\mathrm{qPE}$ cuts can be made at the 
beginning. The code then loops through all the blocks of all the PMTs that observe charge in the event and a calibrated waveform is constructed. After this a $176 \mathrm{~ns}$ wide rolling integral will scan over a the waveform (binned in $4 \mathrm{~ns}$ bins). If the value of the integral is greater then the user set threshold then the time is pushed back into a vector. In order to ensure that all the peaks are unique pulses, the trigger time vector is looped over with the condition that if the difference in time between a given time and the previous time in the vector is greater then $4 \mathrm{~ns}$ (the bin width), the event will be classified as a unique event. Unique events are then pushed back into a new vector. The trigger time values for unique events are then written out to the variable trigTimeLowThresh that can be used during the data analysis. There is also a calcut flag (0x10000) that is set throughout the processor to indicate if an event passes the processor (events that have a charge that is greater then the user define threshold). Figure 4.6 is a flow chart representation of the low threshold trigger time processor.

In analysis trigTimeLowThresh is used to ensure that an event will have enough time in the wide integration time window in order to calculate $\mathrm{F}_{\text {prompt }}$ properly. This means that instead of only using well calibrated trigger times, like eventTime, trigTimeLowThresh will allow any waveform that has a main peak with pulse times between $0 \mathrm{~ns}$ and $6000 \mathrm{~ns}$ into the analysis. Since the waveform is $16 \mu \mathrm{s}$, this ensures that there is a least $10 \mu$ s remaining after the main pulse for the full integration window and no waveform information will be truncated by the fixed size of the time window. 


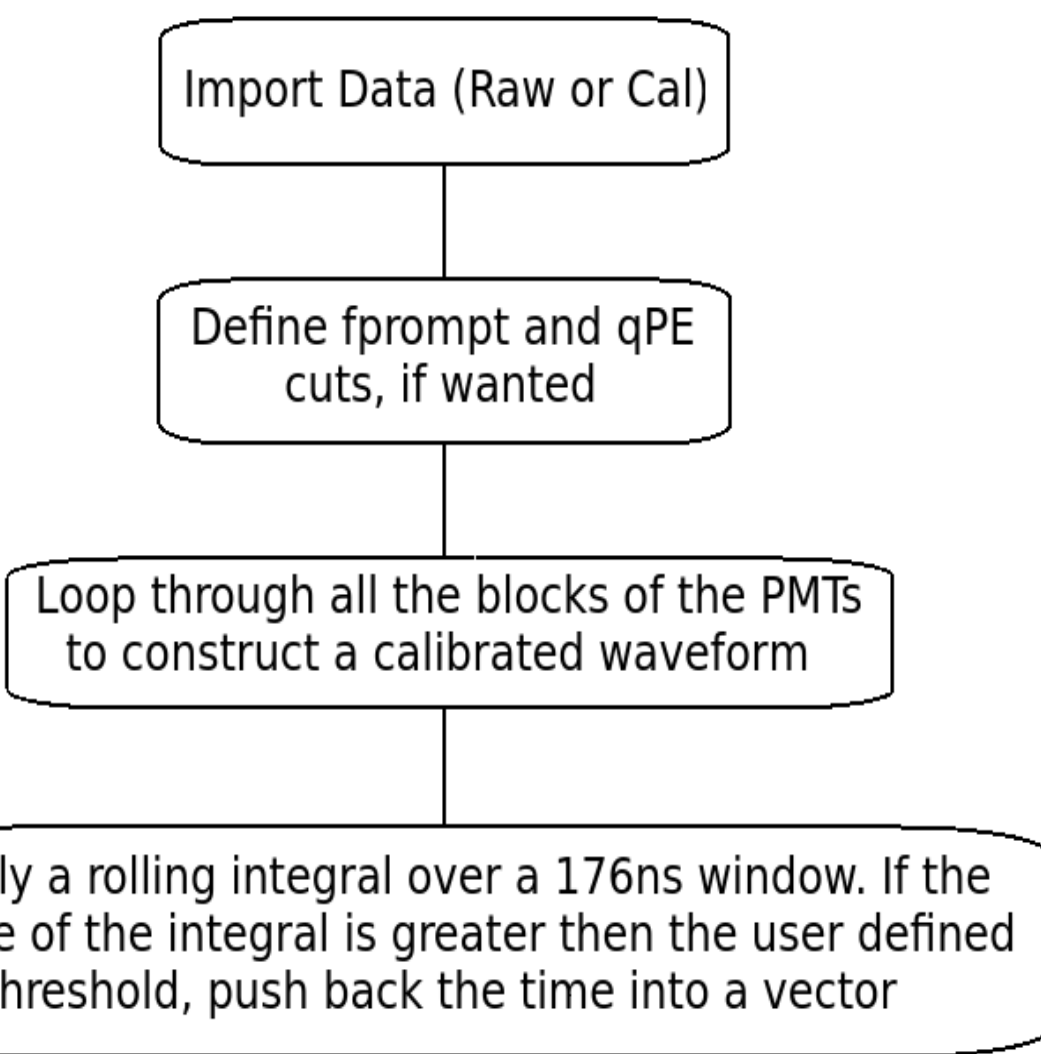

Loop over the times that passed the threshold to verify that all the times correspond to unique pulses. if there is more then one peak, push back the each peak time into a new vector

Apply a rolling integral over a 176 ns window. If the value of the integral is greater then the user defined threshold, push back the time into a vector

Figure 4.6: A flow chart describing how the trigTimeLowThresh variable is calculated using thelow threshold trigger processor. 


\subsection{Low Energy Events}

A particular interest of this analysis is understanding the origin of low energy events. In the context of this analysis a low energy event is defined as an event that has an energy less then $50 \mathrm{PE}$. An example of a low threshold qPE spectrum with pile-up cuts applied can be seen in Figure 4.7. In this comparison the low threshold data and Monte Carlo are both representative of the same detector live time. Both of the sprecta in Figure 4.7 are in agreement with ${ }^{39} \mathrm{Ar}$ recoils above $\sim 100 \mathrm{PE}$.

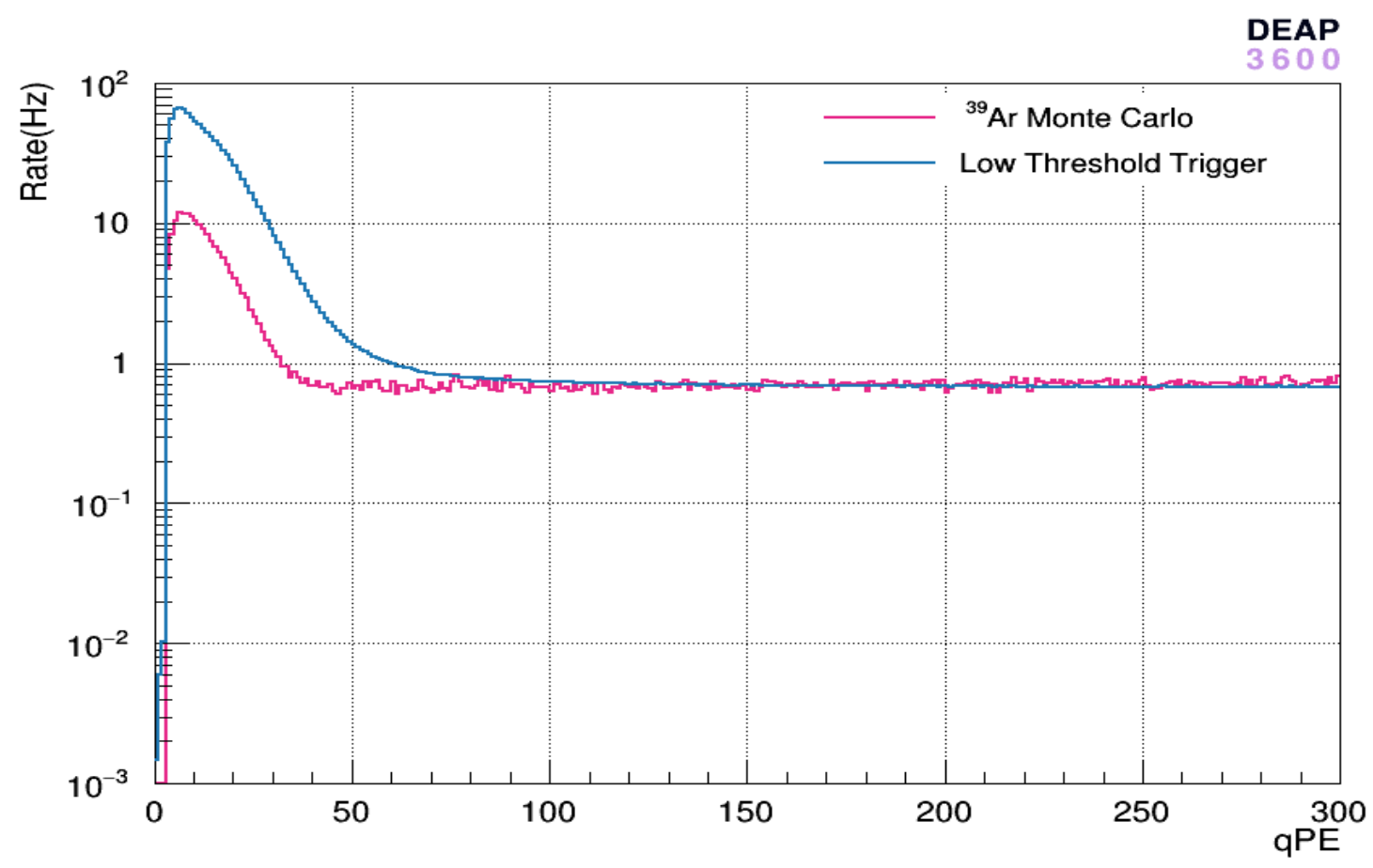

Figure 4.7: A comparison of qPE spectra of low threshold data and low threshold ${ }^{39} \mathrm{Ar}$ Monte Carlo. The spectra agree with one another above $\sim 100 \mathrm{PE}$, so the identification of events below this becomes the main focus.

Low energy events are important to understand in order to get the best sensitivity possible. The number of low energy events that remain in the data for analysis is a determining factor of what the final efficiency will be at low energy, the more low energy events that are kept corresponds to a higher efficiency. Low energy events will also be a dominant factor in the final sensitivity of the low mass WIMP search, espe- 
cially when performing a background subtraction analysis (like the analysis presented in this work). The first step it to understand what is causing these events, whether it is a physical process, a radioactive process or a detector effect. This section will discuss the possible candidates for low energy events and what can be done with them in analysis.

\subsubsection{Retriggers}

Looking at the ${ }^{39} \mathrm{Ar}$ Monte Carlo curve (pink) in Figure 4.7, notice that there is a low energy peak centered at $\sim 15 \mathrm{qPE}$ which is uncharacteristic of ${ }^{39} \mathrm{Ar}$ recoils. The curve that is used to generate the ${ }^{39}$ Ar recoils in Monte Carlo is shown in Figure 4.8 and this low energy peak is not present. Resolving the origin of this peak is crucial to be able to probe low energy events since it lays in the low energy region that is being investigated.

Monte Carlo information provides detailed information about all of the events that take place during the simulation that was run. The Monte Carlo variables of interest are mc_id, the event identification number that is assigned by the simulation; mc_r, the radial position in the detector where the event is initiated by the simulation; mblikelihoodR (Section 4.1.7); qPE (Section 4.1.3); and deltaT (Section 4.1.5). Looking at the information in Figure 4.9, there are multiple events with the same mc_id value. Having multiple events with the same mc_id indicates that all of these events correspond to the same initial event, with all secondary events being retriggers. mblikelihoodR shows that all of the secondary events reconstruct near the surface of the AV with very low energy (small qPE) and occur a long time after the initial event (large deltaT). Results from this investigation show that the DAQ is being retriggered many micro seconds after the initial trigger. 


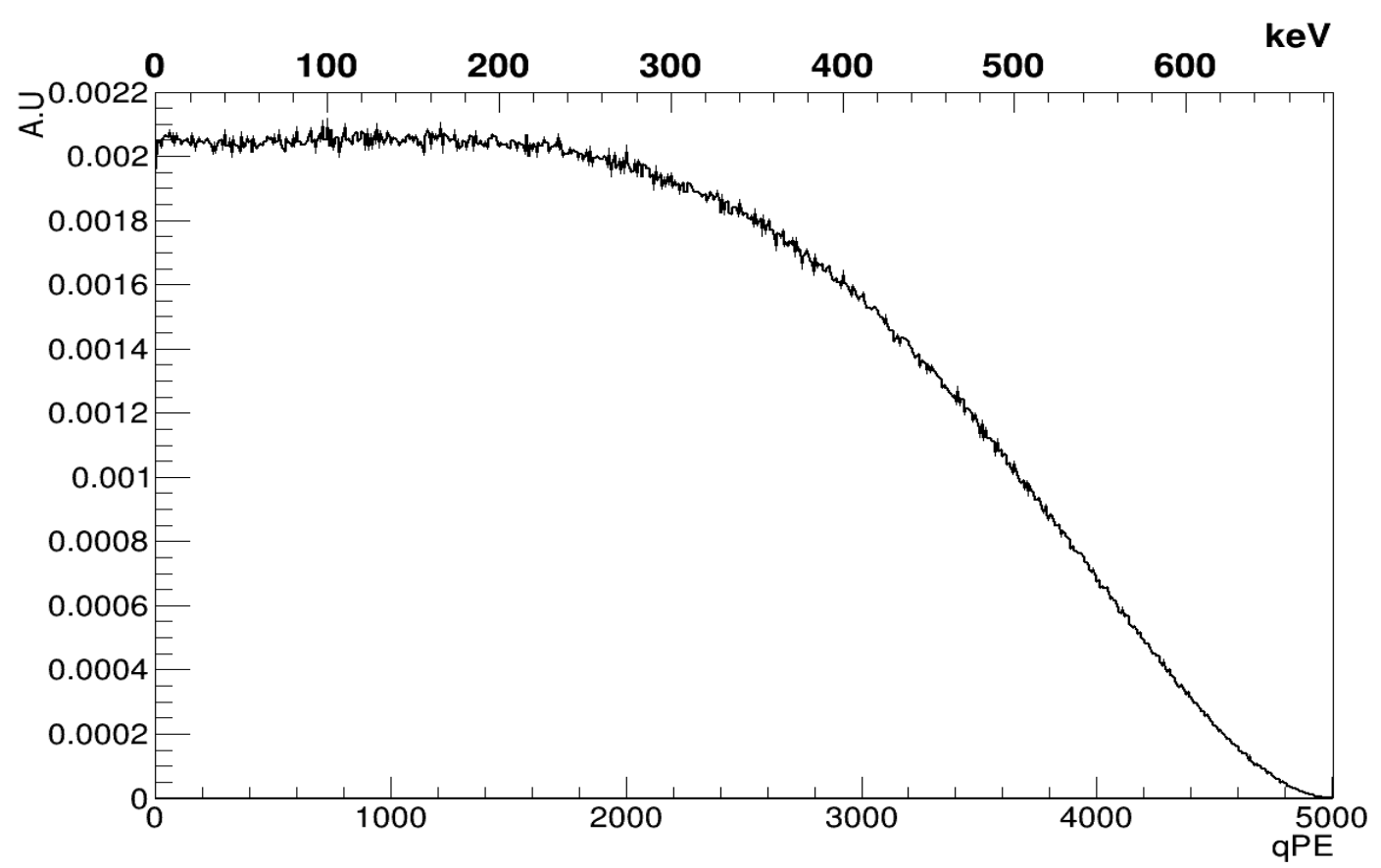

Figure 4.8: ${ }^{39} \mathrm{Ar}$ spectrum that is used by the DEAP collaboration to produce ${ }^{39} \mathrm{Ar}$ Monte Carlo.

In order to investigate if this is the only cause of the low energy peak in the ${ }^{39} \mathrm{Ar}$ Monte Carlo, an analysis was performed such that, if an event had multiple subevents under the same mc_id value, all of the secondary events are removed. The same qPE histogram is plotted for all of the initial events and a second histogram is also plotted for all of the secondary events (retriggers), seen in Figure 4.10a and 4.10b respectively. Based on the results of this investigation it is clear that retriggers are the only cause of the low energy peak in the ${ }^{39}$ Ar Monte Carlo.

There is no event level information like mc_id in regular data, so a different method for the removal of these events had to be developed. Based off the scan information a logical choice would be to use mblikelihoodR, since the retriggers preferentially reconstruct near the AV surface. The other variable that makes sense to use is $\mathrm{F}_{\operatorname{maxPE}}$; since these events are very low energy and they are happening near the surface of the AV a lot of the light will be observed by a small fraction of PMTs meaning that retrigger events would have high $\mathrm{F}_{\operatorname{maxPE}}$ values. 


$\begin{array}{lrrrrr}* & \text { Row } & \text { MC id } * \text { mC_r } & \text { mblikelih } * & \text { qPE * } & \text { deltat } * \\ * * * * * * * * * * * * * * * * * * * * * * * * * * * * * * * * * * * * * * * * * * * * * * * * * * * * * * * * * * * * \\ * & 0 * & 0 * 850.46539 * 847.18011 * 1099.9492 * & 0 * \\ * & 1 * & 1 * 703.55547 * 705.38140 * 1941.9514 * & 0 * \\ * & 2 * & 1 * 703.55547 * 849.77233 * 16.404876 * & 19712 * \\ * & 3 * & 1 * 703.55547 * 849.96728 * 5.3708095 * & 21824 * \\ * & 4 * & 2 * 783.17930 * 772.36529 * 648.00146 * & -41472 * \\ * & 5 * & 3 * 285.29836 * 285.92459 * 1365.4370 * & -63 * \\ * & 6 * & 3 * 285.29836 * 849.98779 * 4.6495122 * & 27936 * \\ * & 7 * & 4 * 307.71732 * 323.27664 * 3662.9668 * & -27951 *\end{array}$

Figure 4.9: Scan of Monte Carlo data looking for a pattern to low energy events. All of the low energy events are retriggers to intial events with the same mc_id.

A study was performed using the ${ }^{39} \mathrm{Ar}$ Monte Carlo data to help motivate an appropriate mblikelihoodR and $\mathrm{F}_{\operatorname{maxPE}}$ cut selection that can be used to remove retriggers. The results of this study are given in Figure 4.11 (these plots also include subeventN, numEarlyPulses and eventTime cuts). Figure 4.11 shows that a cut of mblikelihoodR $<800$ and $\mathrm{F}_{\operatorname{maxPE}}<0.2$ will remove most of the retrigger events in the data. If the number of retrigger events that are removed by this cut are counted and compared to the total number of retriggers, $99.84 \%$ of the retriggers are removed. 


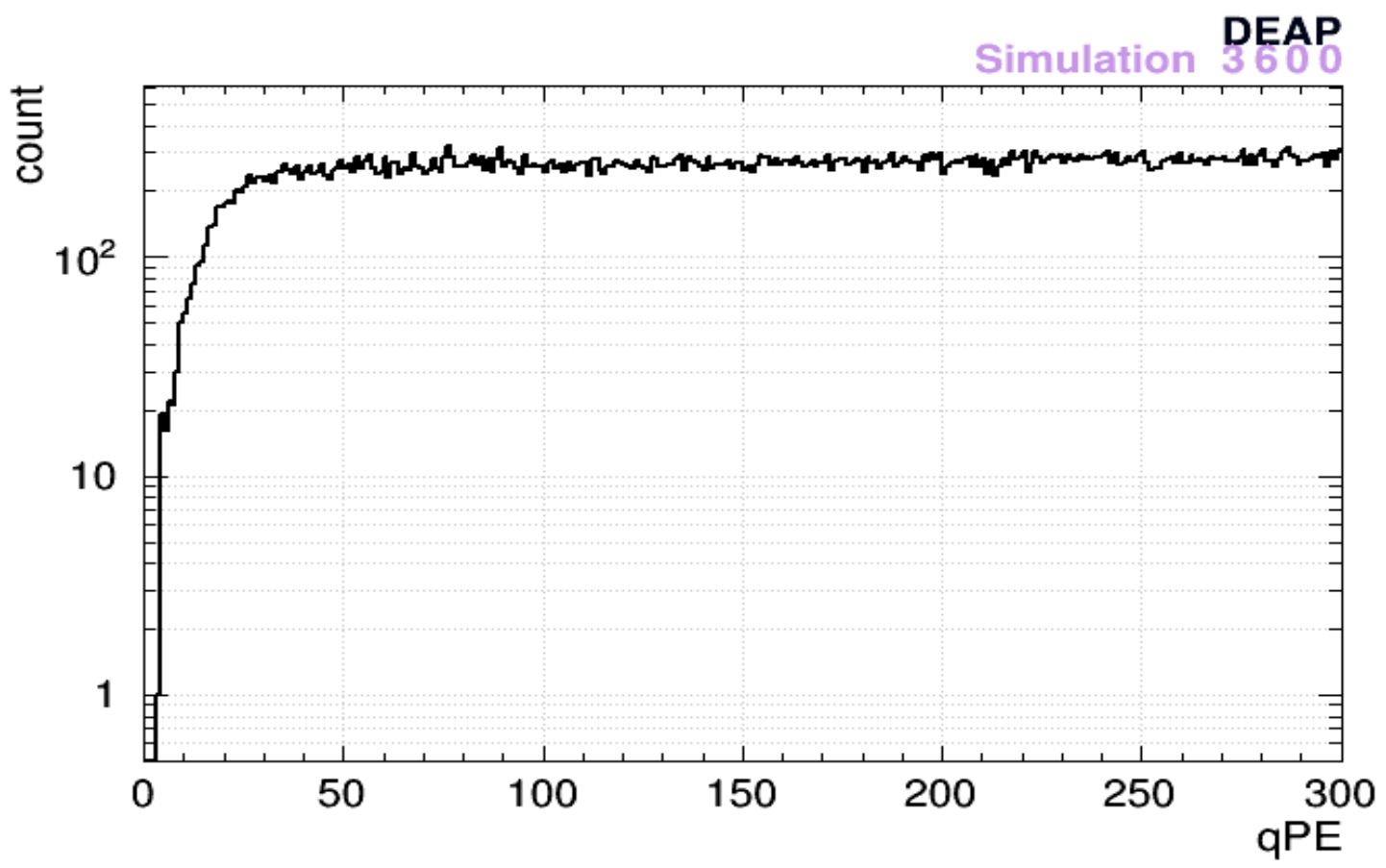

(a) ${ }^{39} \mathrm{Ar}$ Monte Carlo qPE spectrum with all of the secondary events (retriggers) removed.

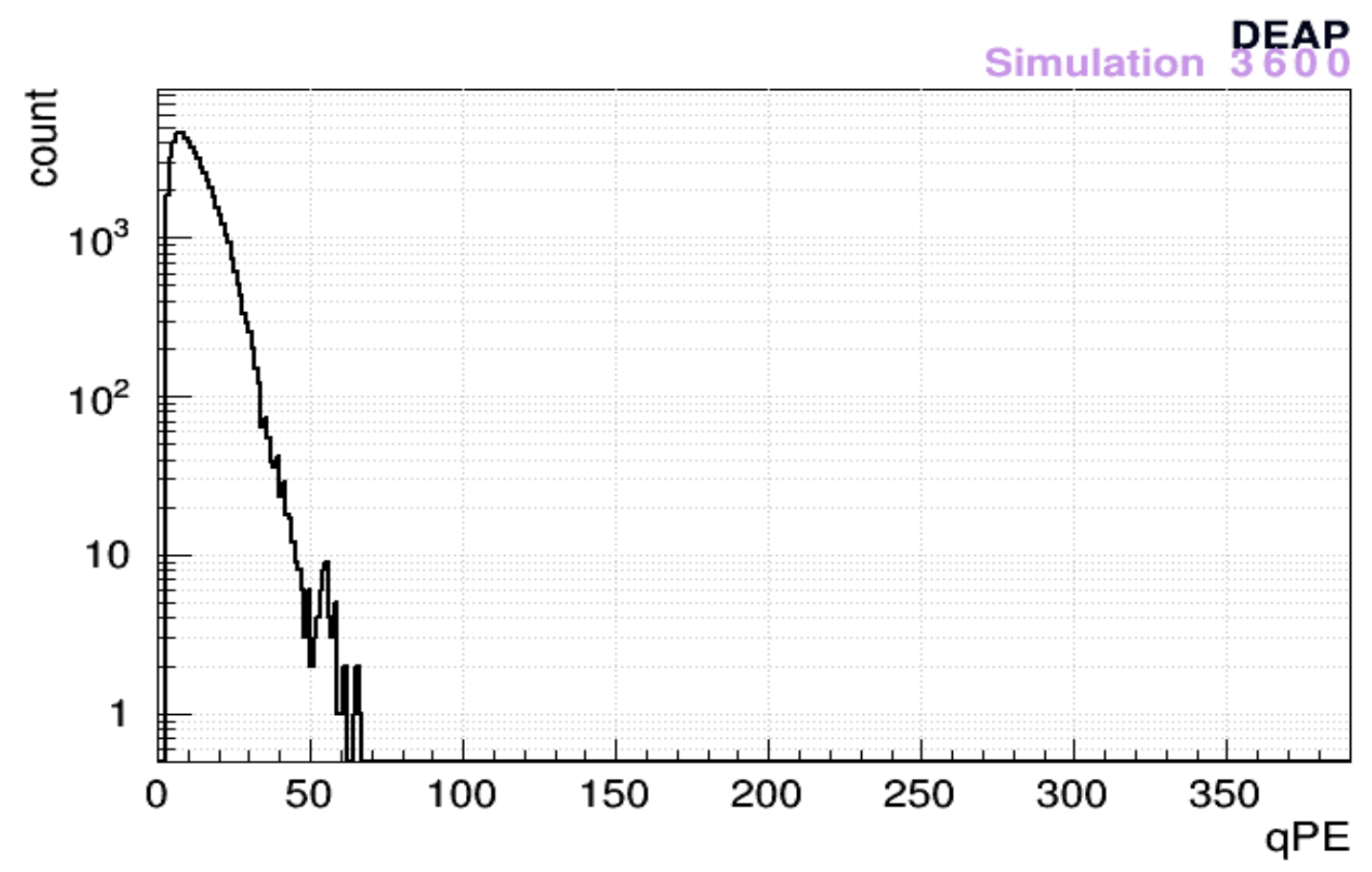

(b) ${ }^{39}$ Ar Monte Carlo qPE spectrum of all of the secondary events (retriggers).

Figure 4.10: Investigating the cause of the low energy peak in ${ }^{39} \mathrm{Ar}$ Monte Carlo. The low energy peak can be explained by retriggers that happen many microseconds after the initial event. 


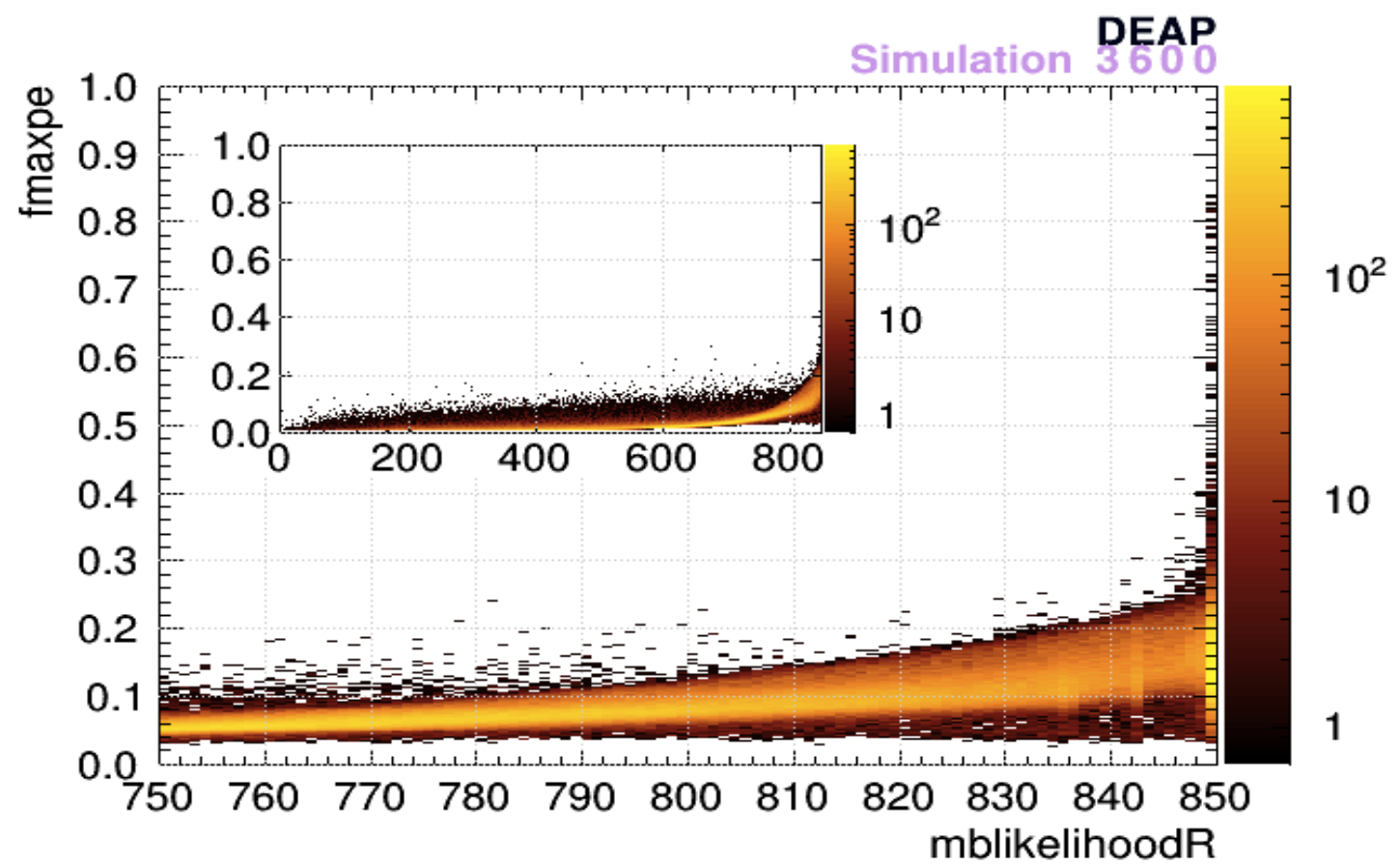

(a) ${ }^{39}$ Ar Monte Carlo with all of the secondary events (retriggers) removed.

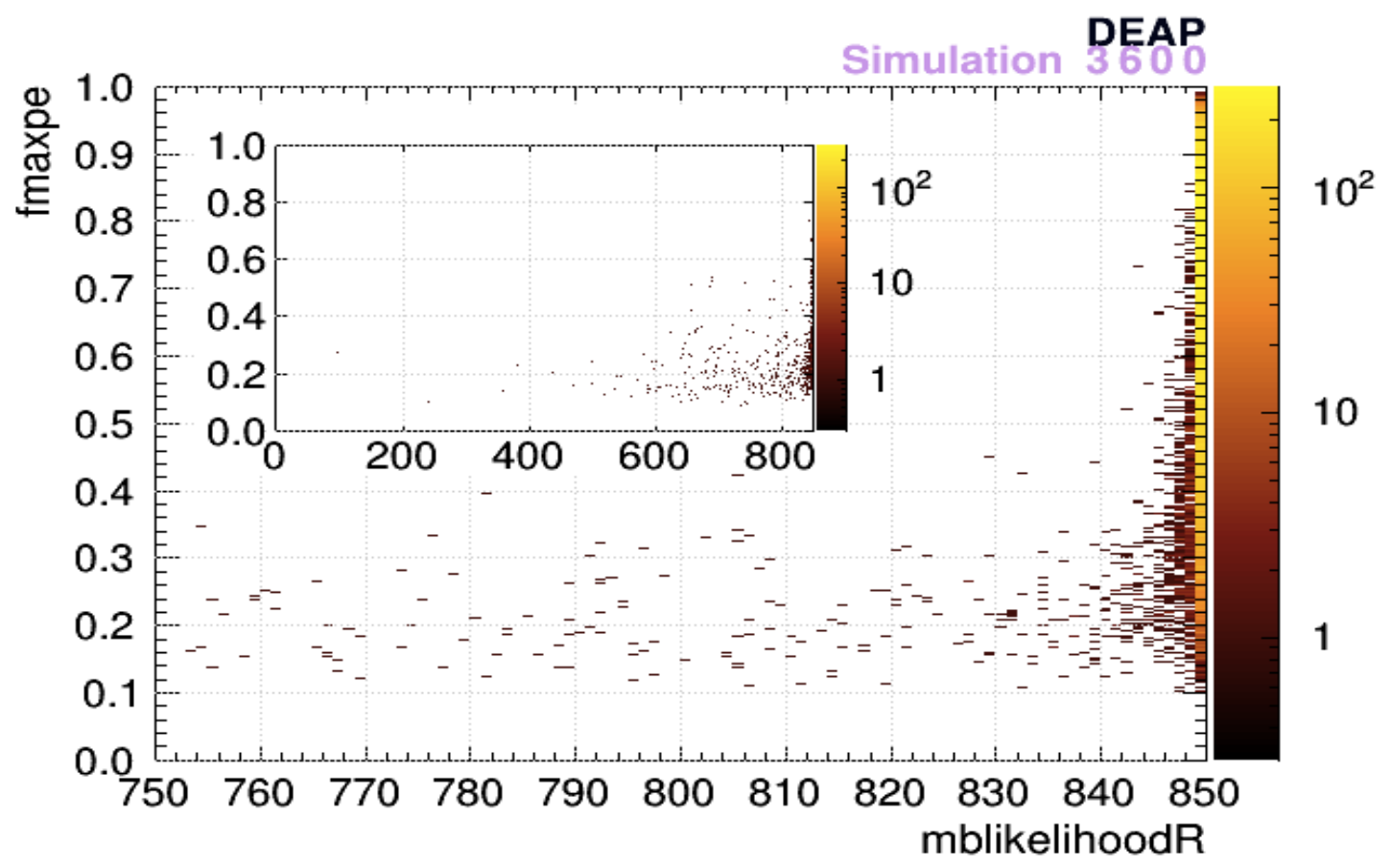

(b) ${ }^{39} \mathrm{Ar}$ Monte Carlo of all of the secondary events (retriggers) only.

Figure 4.11: Investigating ${ }^{39} \mathrm{Ar}$ Monte Carlo to help inform appropriate mblikelihoodR and $\mathrm{F}_{\operatorname{maxPE}}$ cuts. Based on Figure $\mathrm{b}$, performing a cut of mblikelihoodR $<800$ and $\mathrm{F}_{\operatorname{maxPE}}<0.2$ will remove $99.84 \%$ of retriggers. 
Figure 4.11 a shows that there is a large population of non-retriggers that occur near the AV surface at values of mblikelihoodR $>800$ and $\mathrm{F}_{\operatorname{maxPE}}>0.2$. If the fraction of events that are removed by this set of cuts is calculated, $29.8 \%$ of events are removed. This does not include a deltaT cut. However, if the fraction of events that is removed by a deltaT cut of deltaT $>60000 \mathrm{~ns}$ is worked out analytically (based on the low threshold data), it is found that an additional $29 \%$ of the events is removed. deltaT has to be calculated analytically for the Monte Carlo because the ${ }^{39} \mathrm{Ar}$ events are not specifically simulated at a rate that is the same as in data (a rate of $\sim 3115 \mathrm{~Hz}$ ). After all of the pile-up cuts, a eventTime cut as well as the mblikelihoodR and $\mathrm{F}_{\operatorname{maxPE}}$ cuts, $50.14 \%$ of the events will be removed. Given that $50.14 \%$ of events are removed means that the overall cut efficiency of all the background removal cuts is $49.86 \%$ based on the ${ }^{39}$ Ar Monte Carlo.

\subsubsection{Cherenkov Events}

Retriggers are only a partial contribution to the low energy peak in data seen in Figure 4.7. Another possible low energy background that can make up the remainder of the peak is Cherenkov radiation (Section 2.5.2). Cherenkov radiation in DEAP3600 is investigated by performing Monte Carlo simulations of the radioactive decays of ${ }^{40} \mathrm{~K}$ and ${ }^{232} \mathrm{Th}$ in the LGs and PMT glass as well as ${ }^{238} \mathrm{U}$ in the PMT glass only. As mentioned in Section 2.5.2 an electron (or gamma) must have an energy of at least $126.1 \mathrm{keV}$ in order to produce Cherenkov radiation in PMT glass and $174.6 \mathrm{keV}$ to produce Cherenkov radiation in PMMA. A summary of the ${ }^{232}$ Th decay chain is given in Table 4.3. Based on this information the only decay progeny that will contribute to producing Cherenkov light are ${ }^{228} \mathrm{Ac},{ }^{212} \mathrm{~Pb},{ }^{212} \mathrm{Bi}$, and ${ }^{208} \mathrm{Tl}$. The qPE spectra of Cherenkov radiation produced by ${ }^{232} \mathrm{Th}$ in PMT glass and in the LGs can be seen in Figure 4.12 and Figure 4.13 respectively. Pile-up cuts, eventTime cut, and a $\mathrm{F}_{\text {prompt }}<0.6$ cut are applied to all of the following figures in this section. The $\mathrm{F}_{\text {prompt }}$ 
cut separates Cherenkov radiation from events caused by gammas entering the AV.

\begin{tabular}{c|c|c|c|c}
\hline Element & Decay Type & Half Life & Q-Value (keV) & Branching (\%) \\
\hline \hline${ }^{232} \mathrm{Th}$ & $\alpha$ & $1.41 \times 10^{10} \mathrm{yrs}$ & 4082.814 & 100 \\
${ }^{228} \mathrm{Ra}$ & $\beta$ & $5.7 \mathrm{yrs}$ & 45.99 & 100 \\
${ }^{228} \mathrm{Ac}$ & $\beta$ & $6.1 \mathrm{hrs}$ & 21273 & 100 \\
${ }^{228} \mathrm{Th}$ & $\alpha$ & $1.9 \mathrm{yrs}$ & 5520.122 & 100 \\
${ }^{224} \mathrm{Ra}$ & $\alpha$ & 3.6 days & 5788.872 & 100 \\
${ }^{220} \mathrm{Rn}$ & $\alpha$ & $55 \mathrm{~s}$ & 6404.671 & 100 \\
${ }^{216} \mathrm{Po}$ & $\alpha$ & $0.14 \mathrm{~s}$ & 6906.55 & 100 \\
${ }^{212} \mathrm{~Pb}$ & $\beta$ & $10.6 \mathrm{hrs}$ & 573.820 & 100 \\
${ }^{212} \mathrm{Bi}$ & $\alpha$ & $61 \mathrm{mins}$ & 6207.144 & 35.94 \\
${ }^{212} \mathrm{Bi}$ & $\beta$ & $61 \mathrm{mins}$ & 2254.017 & 64.06 \\
${ }^{212} \mathrm{Po}$ & $\alpha$ & $3 \times 10^{-7} \mathrm{~s}$ & 8954.131 & 100 \\
${ }^{208} \mathrm{Tl}$ & $\beta$ & $3.1 \mathrm{mins}$ & 5001.017 & 100 \\
\hline
\end{tabular}

Table 4.3: Decay information for ${ }^{232} \mathrm{Th}$ and all of it progeny. Based on the information the main contributors to Cherenkov radiation will be ${ }^{228} \mathrm{Ac},{ }^{212} \mathrm{~Pb},{ }^{212} \mathrm{Bi}$, and ${ }^{208} \mathrm{Tl}[50]$.

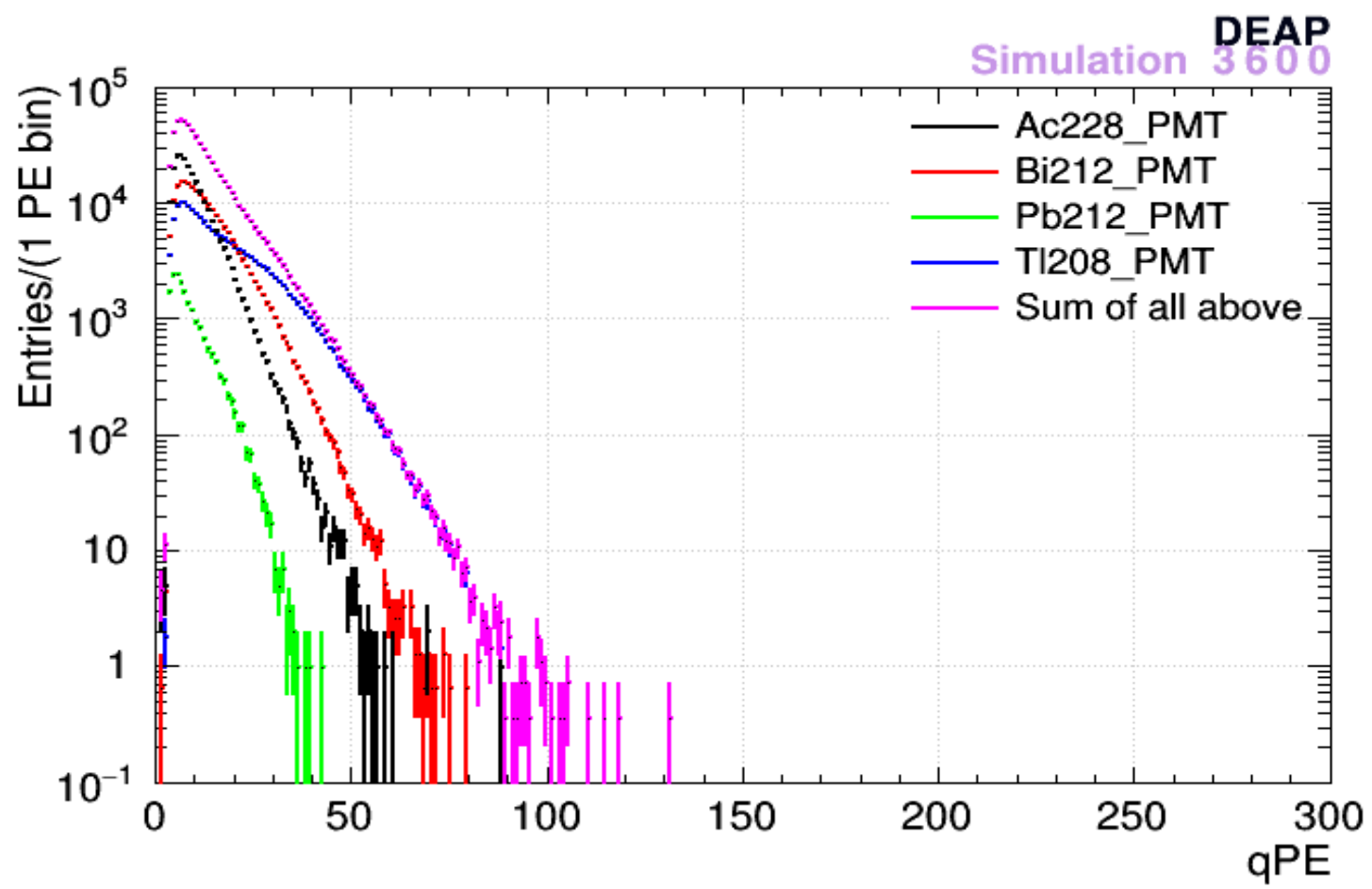

Figure 4.12: Cherenkov radiation contribution from ${ }^{232} \mathrm{Th}$ in PMT glass. The sum of all the progeny contributions can be seen in magenta. 


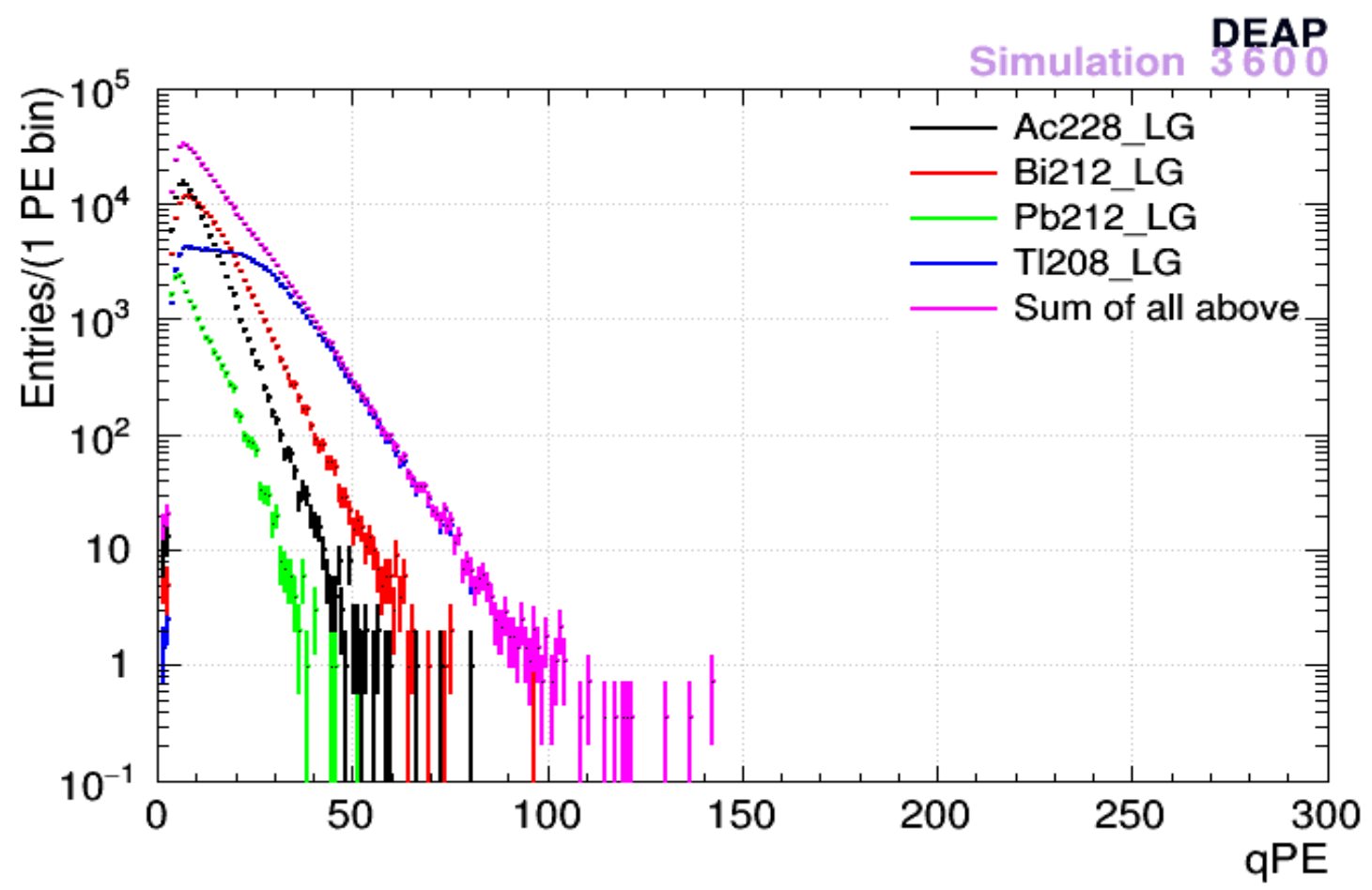

Figure 4.13: Cherenkov radiation contribution from ${ }^{232} \mathrm{Th}$ in the LGs. The sum of all the progeny contributions can be seen in magenta.

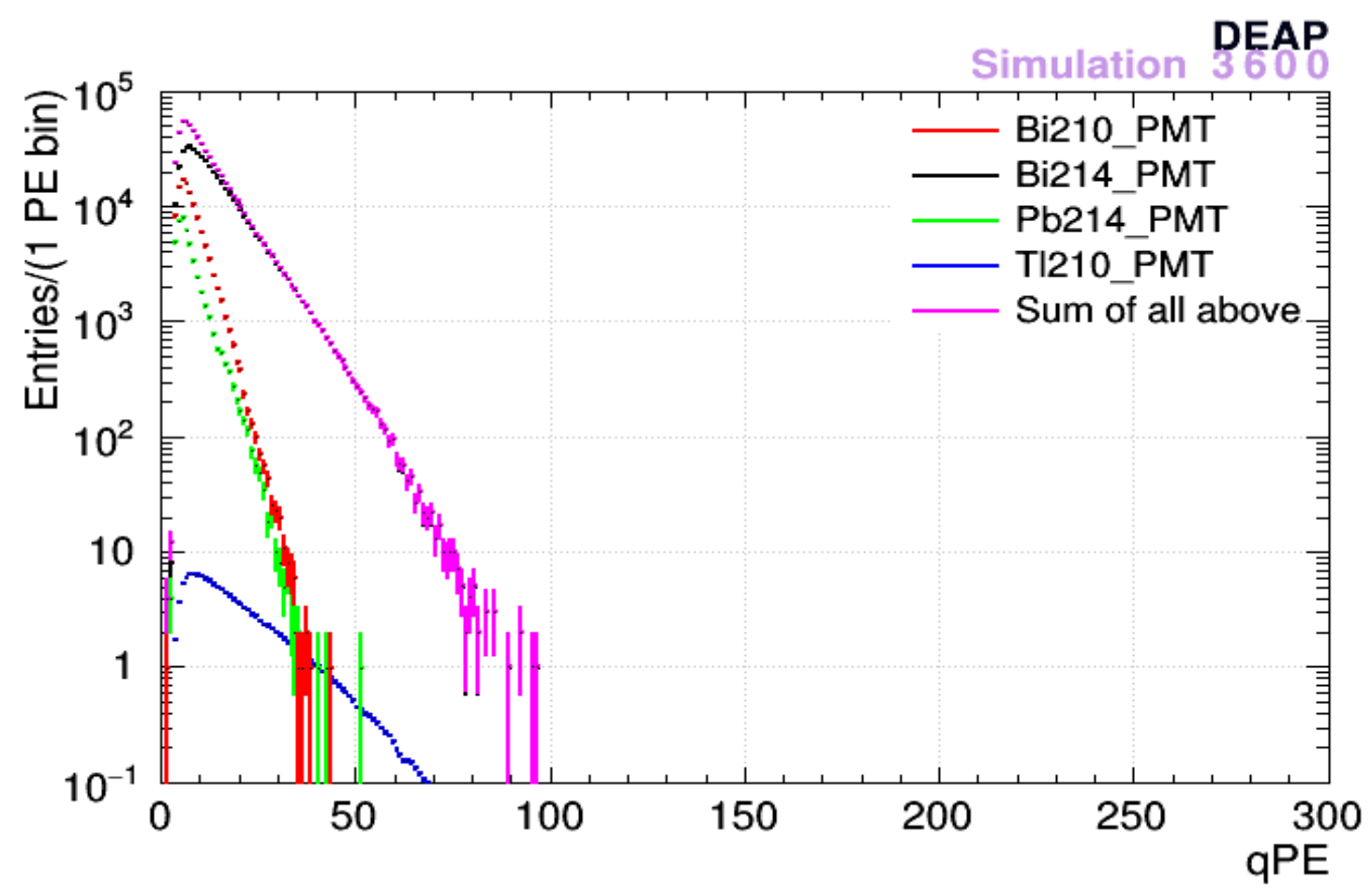

Figure 4.14: Cherenkov radiation contribution from ${ }^{238} \mathrm{U}$ in the PMT glass. The sum of all the progeny contributions can be seen in magenta. 
Table 4.4 indicates that only ${ }^{238} \mathrm{U}$ progeny that will contribute to the Cherenkov radiation from the PMT glass are ${ }^{214} \mathrm{Bi},{ }^{214} \mathrm{~Pb},{ }^{210} \mathrm{Tl}$, and ${ }^{210} \mathrm{Bi}$. It would appear that ${ }^{206} \mathrm{Hg}$ and ${ }^{206} \mathrm{Tl}$ should also contribute due to their high q-values, but these are heavily suppressed by the very small $\alpha$-decay branching fractions of ${ }^{210} \mathrm{~Pb}$ and ${ }^{210} \mathrm{Bi}$ respectively. For the purposes of this study, ${ }^{206} \mathrm{Hg}$ and ${ }^{206} \mathrm{Tl}$ contributions are considered to be negligible. ${ }^{234} \mathrm{Th}$ and ${ }^{234} \mathrm{~Pa}$ also have high q-values, but are both suppressed by the very long half life of ${ }^{238} \mathrm{U}$ and are also considered negligible in this study. The qPE spectra of the total contribution of Cherenkov radiation from ${ }^{238} \mathrm{U}$ in PMT glass can is showm in Figure 4.14

\begin{tabular}{c|c|c|c|c}
\hline Element & Decay Type & Half Life & Q-Value $(\mathrm{keV})$ & Branching $(\%)$ \\
\hline \hline${ }^{238} \mathrm{U}$ & $\alpha$ & $4.468 \times 10^{9} \mathrm{yrs}$ & 42703 & 100 \\
${ }^{234} \mathrm{Th}$ & $\beta$ & 24.10 days & 2733 & 100 \\
${ }^{234} \mathrm{~Pa}$ & $\beta$ & $6.70 \mathrm{hrs}$ & 21955 & 100 \\
${ }^{234} \mathrm{U}$ & $\alpha$ & $2.455 \times 10^{5} \mathrm{yrs}$ & 4858.57 & 100 \\
${ }^{230} \mathrm{Th}$ & $\alpha$ & $7.538 \times 10^{4} \mathrm{yrs}$ & 4770.02 & 100 \\
${ }^{226} \mathrm{Ra}$ & $\alpha$ & $1600 \mathrm{yrs}$ & 4870.63 & 100 \\
${ }^{222} \mathrm{Rn}$ & $\alpha$ & 3.823 days & 5590.33 & 100 \\
${ }^{218} \mathrm{Po}$ & $\alpha$ & $3.10 \mathrm{mins}$ & 6114.69 & 99.98 \\
${ }^{218} \mathrm{Po}$ & $\beta$ & $3.10 \mathrm{mins}$ & 26512 & 0.02 \\
${ }^{218} \mathrm{At}$ & $\alpha$ & $1.5 \mathrm{~s}$ & 68743 & 100 \\
${ }^{214} \mathrm{~Pb}$ & $\beta$ & $26.8 \mathrm{mins}$ & 102411 & 100 \\
${ }^{214} \mathrm{Bi}$ & $\alpha$ & $19.9 \mathrm{mins}$ & 5616.81 & 0.02 \\
${ }^{214} \mathrm{Bi}$ & $\beta$ & $19.9 \mathrm{mins}$ & 327211 & 99.98 \\
${ }^{214} \mathrm{Po}$ & $\alpha$ & $164.3 \mathrm{\mu s}$ & 7833.46 & 100 \\
${ }^{210} \mathrm{Tl}$ & $\beta$ & $1.30 \mathrm{mins}$ & 548911 & 100 \\
${ }^{210} \mathrm{~Pb}$ & $\alpha$ & $22.3 \mathrm{yrs}$ & 379220 & $1.9 \times 10^{-6}$ \\
${ }^{210} \mathrm{~Pb}$ & $\beta$ & $22.3 \mathrm{yrs}$ & 63.55 & 100 \\
${ }^{210} \mathrm{Bi}$ & $\alpha$ & 5.01 days & 5036.08 & $1.32 \times 10^{-4}$ \\
${ }^{210} \mathrm{Bi}$ & $\beta$ & 5.01 days & 1162.18 & 100 \\
${ }^{210} \mathrm{Po}$ & $\alpha$ & 138.38 days & 5407.47 & 100 \\
${ }^{206} \mathrm{Hg}$ & $\beta$ & 8.15 mins & 130720 & 100 \\
${ }^{206} \mathrm{Tl}$ & $\beta$ & $4.20 \mathrm{mins}$ & 1533.57 & 100 \\
\hline
\end{tabular}

Table 4.4: Decay information for ${ }^{238} \mathrm{U}$ and all of it progeny. Based on the information the main contributors to Cherenkov radiation will be ${ }^{214} \mathrm{Bi},{ }^{214} \mathrm{~Pb},{ }^{210} \mathrm{Tl}$, and ${ }^{210} \mathrm{Bi}[50]$. 
${ }^{40} \mathrm{~K}$ has a more straight forward decay with the products being either ${ }^{40} \mathrm{Ar}$ or ${ }^{40} \mathrm{Ca} .{ }^{40} \mathrm{Ar}$ is produced through $\gamma$-decay or electron capture that produce $1.51 \mathrm{MeV}$ of energy and has a branching fraction of $10.66 \% .{ }^{40} \mathrm{Ca}$ is produced through $\beta$-decay that produces $1.31 \mathrm{MeV}$ of energy and has a branching fraction of $89.14 \%$. Both of these decays are capable of producing Cherenkov radiation in both the PMT glass and the acrylic. The qPE spectra of Cherenkov produced by ${ }^{40} \mathrm{~K}$ in the PMT glass and in the LGs are shown in Figure 4.15 and Figure 4.16 respectively.

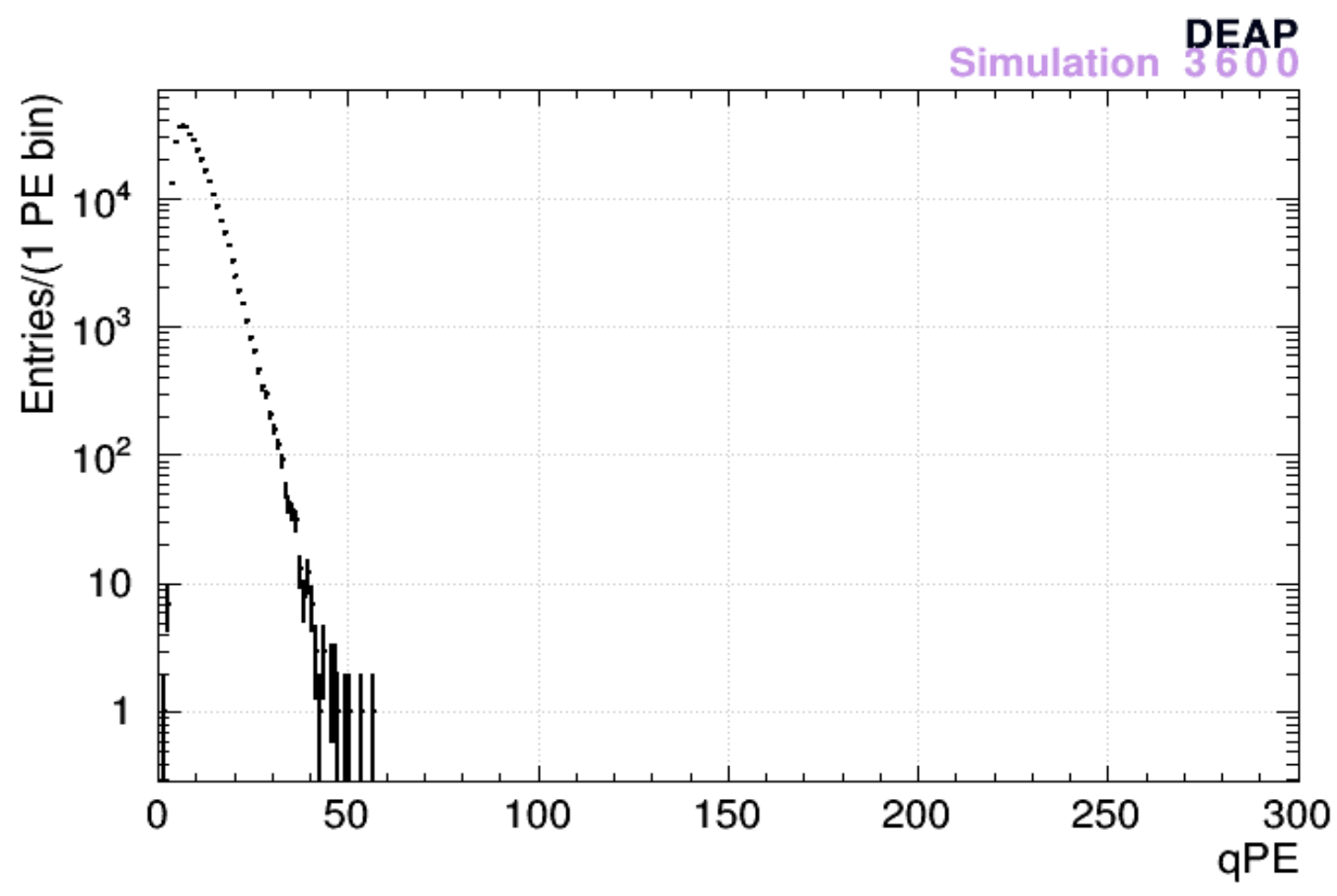

Figure 4.15: Cherenkov radiation contribution from ${ }^{40} \mathrm{~K}$ in the PMT glass.

To get the total Cherenkov rate based on all of the decays discussed above, the histograms can be scaled by their respective branching ratios and then by the rate of decay that has been measured in DEAP-3600 [51]. The sum of all of the contributions normalized to the rate can be seen in Figure 4.17. The only cuts that are applied to this plot are calcut, dtmTrigSrc, pile-up cuts and an eventTime cut. This is to see if the peak can be fully explained with a combination retriggers and Cherenkov alone. Notice that there is a shoulder in the low threshold data (green) curve at around 
$20 \mathrm{qPE}$ that is not present in the Monte Carlo. This was well studied as part of this work, and the conclusion is that these event are produced by the retriggers of very high energy events in the detector. The initial events of these retriggers can not be seen in the data since the DAQ is only recording low energy events. This shoulder is not seen in the Monte Carlo because there are no high energy events being simulated.

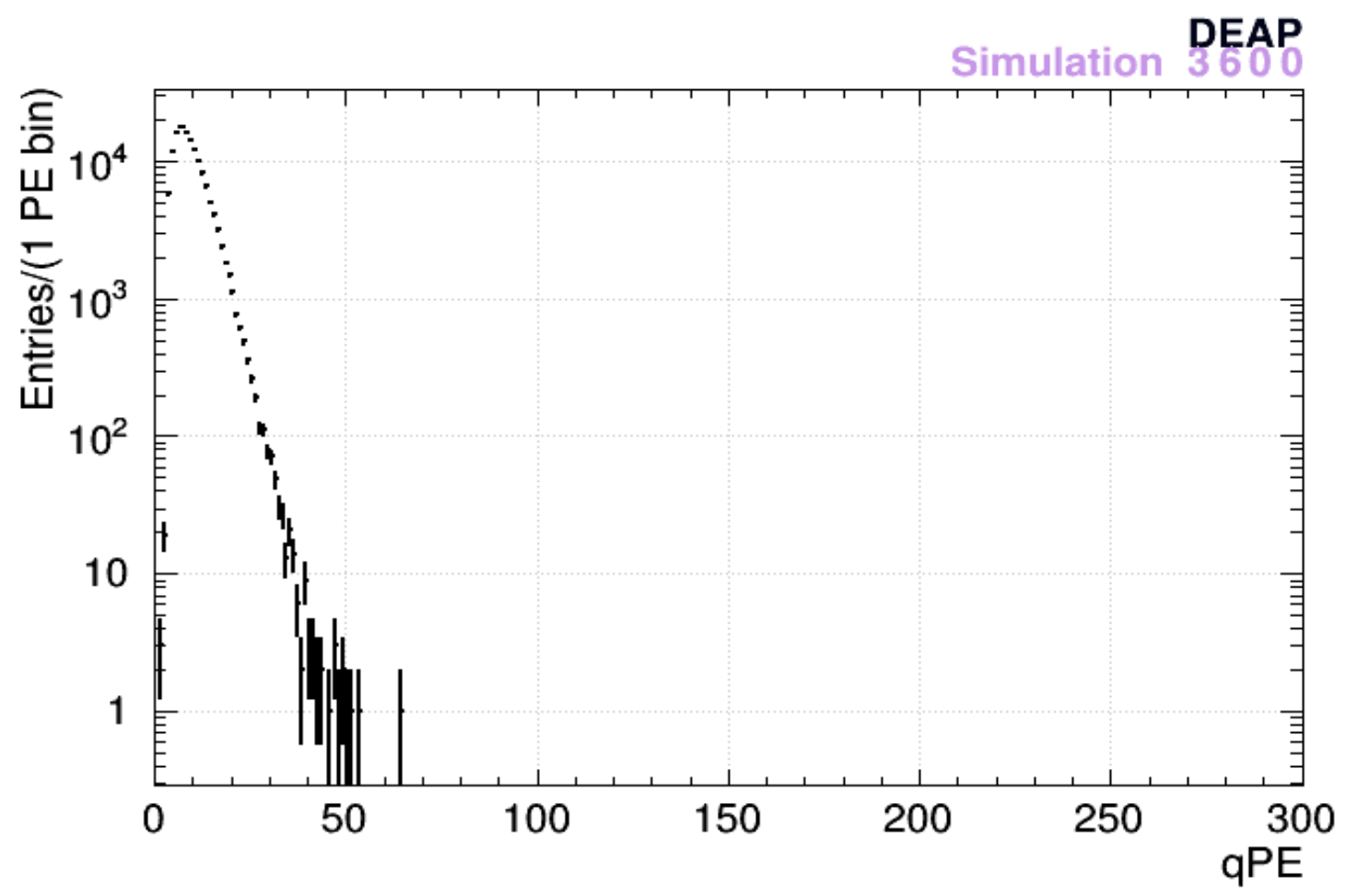

Figure 4.16: Cherenkov radiation contribution from ${ }^{40} \mathrm{~K}$ in the LGs.

A summary of all the data cuts is provided below. The mblikelihoodZ cut is a position reconstruction cut (calculated like mblikelihoodR, but only on the z-axis) that ensures that no events that reconstruct above the LAr fill level are kept in the data set. The same background subtraction was done with all of the cuts applied and the result is shown in Figure 4.18. The low energy peak is removed when all cuts are applied which is expected based on the discussion in Section 4.3.1. There is now agreement between the ${ }^{39} \mathrm{Ar}$ events in data and Monte Carlo. This data set is suitable for conducting a low mass WIMP search through the use of a background subtraction. 
- !(calcut \& 0x31f8) [in data only]

- !(dtmTrigSrc \& 0x82) [in data only]

- subeventN $==1$

- numEarlyPulses $<=3$

- deltat > 60000 [in data only]. Can scale Monte Carlo by 0.71 to mimic cut.

- eventTime $>2300 \& \&$ eventTime $<2650$

- $\mathrm{F}_{\operatorname{maxPE}}<0.2$

- mblikelihoodR $<800$

- mblikelihoodZ $<551$

- $\operatorname{trigTimeLowThresh~}>0$ \&\& trigTimeLowThresh $<6000$

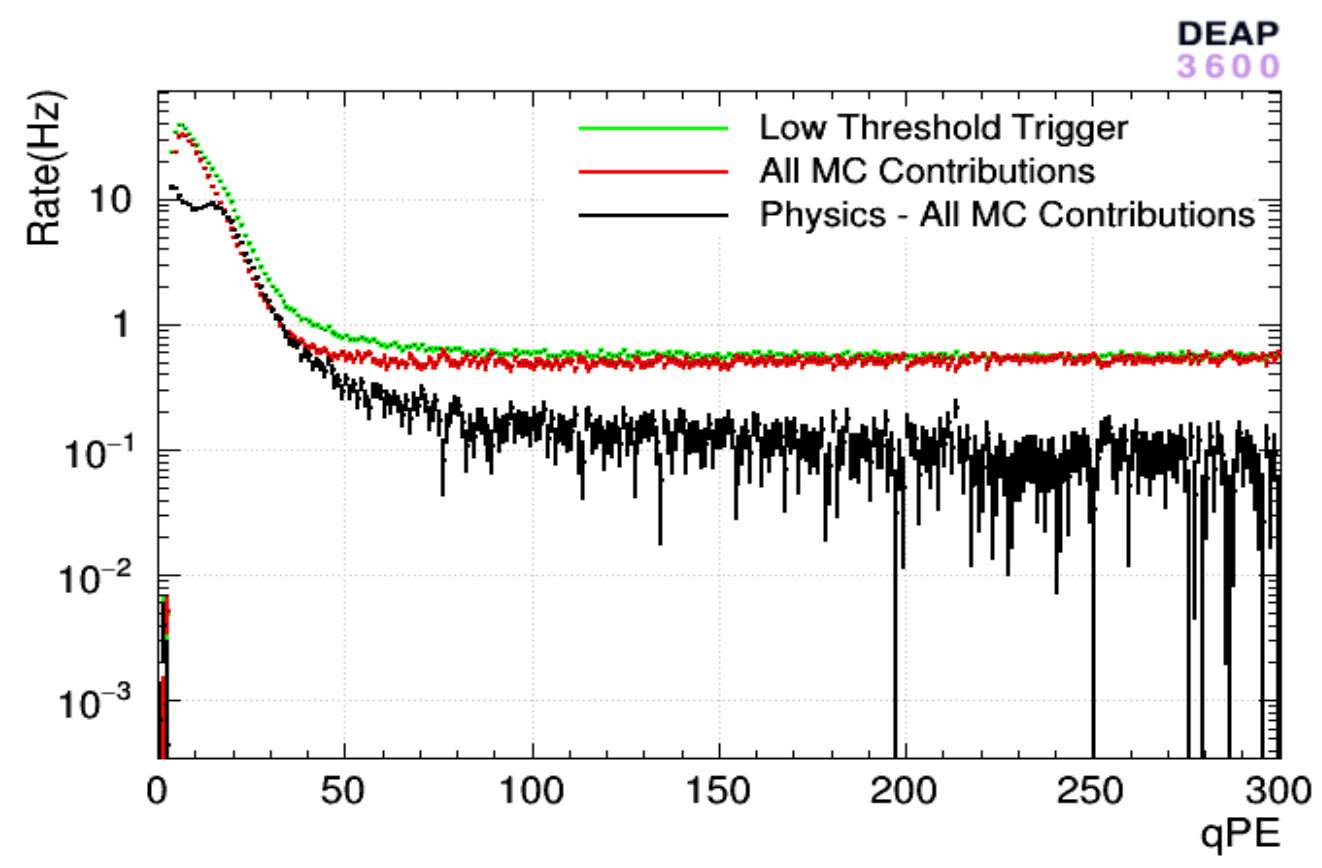

Figure 4.17: Comparison between low threshold data and total Monte Carlo contribution ( ${ }^{39} \mathrm{Ar}$ and all Cherenkov sources) along with a background subtraction seen in black. Pile-up cuts and an eventTime cut are applied. 

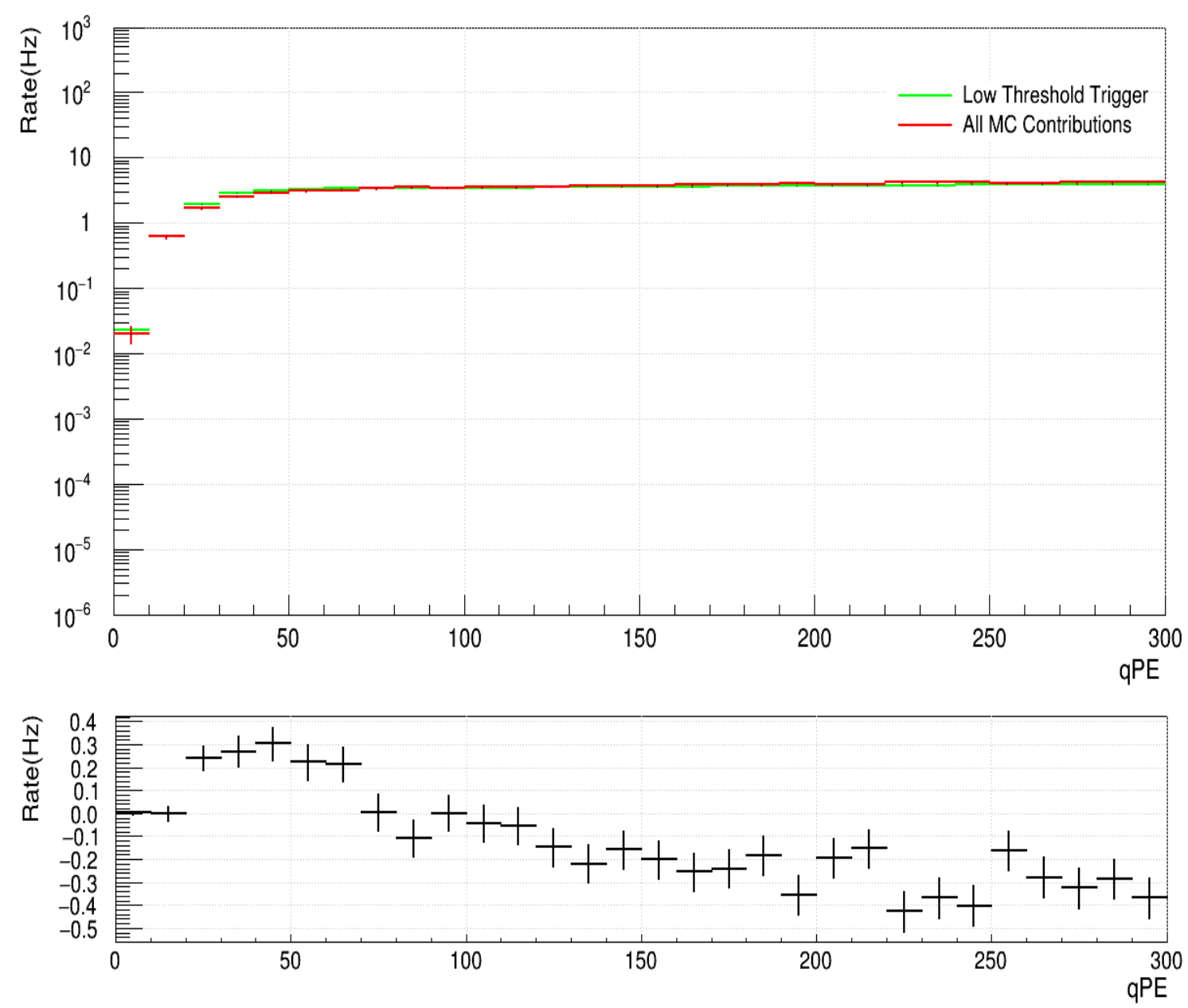

Figure 4.18: Comparison between low threshold data (green) and total Monte Carlo contribution ( ${ }^{39} \mathrm{Ar}$ and all Cherenkov sources) (red) along with a background subtraction seen in black. All cuts are applied. The low energy peak is now gone and there is good agreement between ${ }^{39} \mathrm{Ar}$ events in data and Monte Carlo.

\subsection{Signal Extraction}

The methods for performing the signal extraction in this work will follow the work done by Lewin and Smith in "Review of mathematics. numerical factors, and corrections for dark matter experiments based on elastic nuclear recoil" [52]. The goal of this analysis is to extract enough information from the data in order to construct a WIMP exclusion curve. Pieces for this analysis include a form factor model for ${ }^{40} \mathrm{Ar}$, 
WIMP velocity model, a nuclear recoil model, total nuclear recoil rate in DEAP-3600 and a method for calculating the $90 \%$ upper limit of possible signal events based on the number of background events remaining after background subtraction.

\subsubsection{Nuclear Form Factor}

The nuclear form factor is a parameter which describes how the target nucleus responds to an interaction. Its functional form is given by Equation 4.3

$$
\begin{gathered}
F(q)=3 \frac{j_{1}\left(q r_{N} / \hbar c\right)}{q r_{N} / \hbar c} e^{-\frac{(q s / \hbar c)^{2}}{2}} \\
j_{1}(x)=\frac{\sin (x)-x \cos (x)}{x^{2}} \\
r_{N}=\sqrt{\left(1.23 A^{\frac{1}{3}}-0.60\right)^{2}+\frac{7}{3}(0.52 \pi)^{2}-5 s^{2}},
\end{gathered}
$$

where $j_{1}$ is the first order spherical Bessel function (Equation 4.4). $q$ is the momentum transfer $\left(q=\sqrt{2 M_{N} E_{R}}\right)$ of given a recoil energy, $E_{R}$, and target nucleus mass, $M_{N} \cdot r_{N}$ is the radius of the target nucleus given by Equation 4.5 , where $A=40$, the atomic number, and $s=0.9 \mathrm{fm}$, the nuclear skin thickness.

When the form factor is squared at a given recoil energy it indicates the probability of the event having a coherent nuclear recoil at that energy. The form factor squared of ${ }^{40} \mathrm{Ar}$ for recoil energies up to $1000 \mathrm{keV}_{\mathrm{nr}}$ can be seen in Figure 4.19. The current work goes up to $\sim 140 \mathrm{keV}_{\mathrm{nr}}$, so it does not reach the large drop in probability of coherent nuclear recoils. 


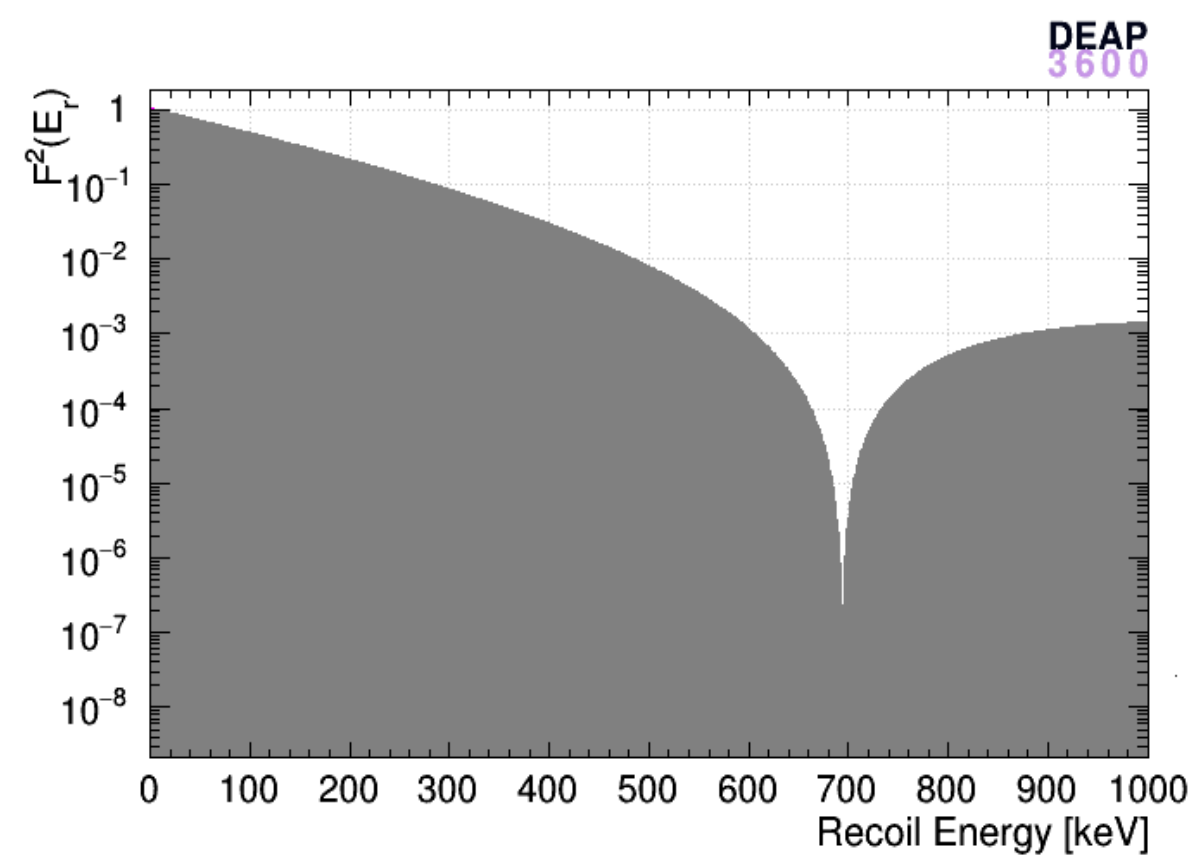

Figure 4.19: The probability of an ${ }^{40} \mathrm{Ar}$ having a coherent nuclear recoil at the given recoil energies. The scope of this work goes up to $\sim 140 \mathrm{keV}_{\mathrm{nr}}$.

\subsubsection{Nuclear Recoil Model}

It is assumed that the WIMP velocity profile follows a Maxwellian distribution given by Equation 4.6 .

$$
f\left(v, v_{E}\right)=e^{-\left(v+v_{E}\right)^{2} / v_{0}^{2}},
$$

where $v$ is the WIMP velocity in the reference frame of the earth, $v_{E}$ is the velocity of the earth in reference to the galaxy, and $v_{0}$ is the mean WIMP velocity with respect to the earth. A normalization constant can be defined as $k$, seen in Equation 4.7 .

$$
k=\int_{0}^{2 \pi} d \phi \int_{-1}^{+1} d(\cos \theta) \int_{0}^{v_{e s c}} f\left(v, v_{E}\right) v^{2} d v .
$$

Using Equation 4.6 and Equation 4.7, two useful normalization constants $\left(k_{0}\right.$ and $\left.k_{1}\right)$ can be calculated for the cases when the escape velocity of the galaxy $\left(v_{e s c}\right)$ is infinite and when it equals the true escape velocity of the galaxy. These are given by 
Equation 4.8 and Equation 4.8 respectively.

$$
\begin{gathered}
k_{0}=\int_{0}^{2 \pi} d \phi \int_{-1}^{+1} d(\cos \theta) \int_{0}^{\infty} f\left(v, v_{E}\right) v^{2} d v=\left(\pi v_{0}^{2}\right)^{\frac{3}{2}} \\
k_{1}=\int_{0}^{2 \pi} d \phi \int_{-1}^{+1} d(\cos \theta) \int_{0}^{v_{e s c}} f\left(v, v_{E}\right) v^{2} d v=k_{0}\left[\operatorname{erf}\left(\frac{v_{e s c}}{v_{0}}\right)-\frac{2}{\sqrt{\pi}} \frac{v_{\text {esc }}}{v_{0}} e^{-v_{\text {esc }}^{2} / v_{0}^{2}}\right] .
\end{gathered}
$$

These normalization constants can then be used to calculate the differential rate of $\left(\frac{d R}{d E_{R}}\right)$ of nuclear recoils.

$$
\begin{gathered}
d R=R_{0} \frac{k_{0}}{k} \frac{1}{2 \pi v_{0}^{4}} v f\left(v, v_{E}\right) d^{3} v \\
R_{0}=\frac{503}{M_{D} M_{T}}\left(\frac{\sigma_{0}}{1 \mathrm{pb}}\right)\left(\frac{\rho_{D}}{0.4 \mathrm{GeVc}^{-2} \mathrm{~cm}^{-3}}\right)\left(\frac{v_{0}}{230 \mathrm{~km} \mathrm{~s}^{-1}}\right) .
\end{gathered}
$$

$R$ is the rate in units of counts $/ \mathrm{kg} /$ day and $E_{R}$ is the nuclear recoil energy. The rate is expressed in terms of the WIMP velocity and can defined as in Equation 4.10. $R_{0}$ is the event rate for $v_{E}=0$ and $v_{e s c}=\infty$ and is defined as Equation 4.11. $M_{D}$ is the WIMP mass, $M_{T}$ is the target nucleus mass, $\sigma_{0}$ is the WIMP-Nucleus cross-section, and $\rho_{D}$ is the local WIMP density.

Using Equation 4.10 the differential rate can be calculated by first determining $\frac{d R\left(v_{E}, \infty\right)}{d E_{R}}$ (Equation 4.12 , which can then be used to determine the true differential rate, $\frac{d R\left(v_{E}, v_{e s c}\right)}{d E_{R}}$ (Equation 4.13 .

$$
\begin{gathered}
\frac{d R\left(v_{E}, \infty\right)}{d E_{R}}=\frac{R_{0}}{E_{0} r} \frac{\pi^{\frac{1}{2}}}{4} \frac{v_{0}}{v_{E}}\left[\operatorname{erf}\left(\frac{v_{\min }+v_{E}}{v_{0}}\right)-\operatorname{erf}\left(\frac{v_{\min }-v_{E}}{v_{0}}\right)\right] \\
\frac{d R\left(v_{E}, v_{e s c}\right)}{d E_{R}}=\frac{k_{0}}{k_{1}}\left[\frac{d R\left(v_{E}, \infty\right)}{d E_{R}}-\frac{R_{0}}{E_{0} r} e^{-v_{e s c}^{2} / v_{0}^{2}}\right]
\end{gathered}
$$




$$
v_{\min }=\left(\frac{E_{R}}{E_{0} r}\right)^{\frac{1}{2}} v_{0}
$$

$v_{\min }$ is the velocity to that corresponds to the $E_{\text {min }}$, the smallest particle energy that can produce a recoil energy $E_{R}$, defined as Equation 4.14. $\mathrm{r}$ is the kinetic term of the momentum transfer.

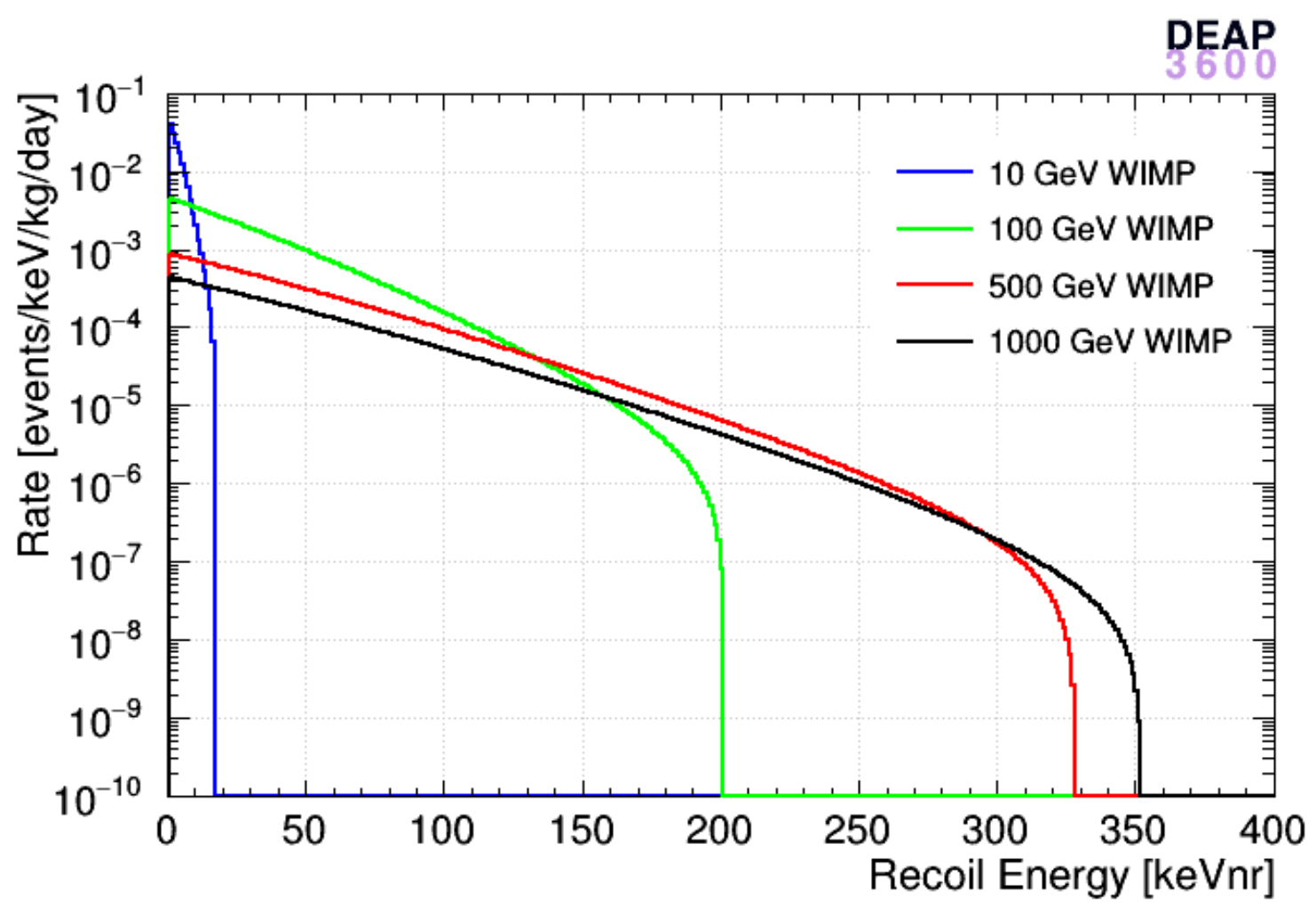

Figure 4.20: The recoil rate spectra for various WIMP masses. Notice as the recoil energy approaches zero, the rate increases exponentially (appears linear in figure due to the log scale of the y-axis).

With this framework, the rate of nuclear recoils based on a given WIMP mass can be determined. An example of nuclear recoil spectra of various WIMP masses is shown in Figure 4.20. The overall trend of the curves shows that there is an exponential increase in the rate of recoils as the recoil energy approaches smaller values. These results provide additional motivation for why reducing the threshold of the detector could be beneficial. Not only does the experiment become more sensitive to lower 
energy events, but the rate of possible nuclear recoils due to a WIMP interaction increases exponentially towards lower energies.

\subsubsection{Total Rate in DEAP-3600}

The information that is provided in Section 4.4 .2 is the way to calculate the rate given a perfect detector. If this rate is multiplied by the exposure of the data set being used and the cut acceptance, a rate with units [events/keV] will be obtained. To convert this information into DEAP-3600 specific rate, the following equation can be used.

$$
E_{M}=E_{R} \times \text { ThresholdCurve }\left(E_{R}\right) \times \text { ResponseFunc }\left(E_{R} \cdot Q F\right) .
$$

$E_{M}$ is the measured energy by the detector, ThresholdCurve $\left(E_{R}\right)$ is an efficiency curve as a function of recoil energy (which is different for every detector), shown Figure 4.21 and $\operatorname{ResponseFunc}\left(E_{R} \cdot Q F\right)$ is a measure of how the detector responds to information at a given energy (dependent on how the detector was built and the setting it is operated at).

The trigger threshold curve is obtained by first, determining the value of the maximum bin of the qPE spectrum of data after cuts. All of the bins in the energy spectrum are divided by this value, providing a relative efficiency for each energy bin. The energy response function in DEAP-3600 is assumed to be a Gaussian distribution with mean, $\mu$ (Equation 4.16), and standard deviation, $\sigma$ (Equation 4.17).

$$
\begin{gathered}
\mu=\left(\left\langle N_{D}\right\rangle+L Y \cdot E_{R}\right) * Q F \\
\sigma^{2}=\sigma_{P E}^{2} \cdot \mu+\sigma_{L Y}^{2} \cdot \mu^{2}
\end{gathered}
$$


$\left\langle N_{D}\right\rangle$ is the average number of PE produced by dark noise, $L Y$ is the light yield, and $Q F$ is the nuclear quenching factor. Once a plot of the measured energy is obtained, the total nuclear recoil rate DEAP-3600 can be calculated by integrating over a desired energy range.

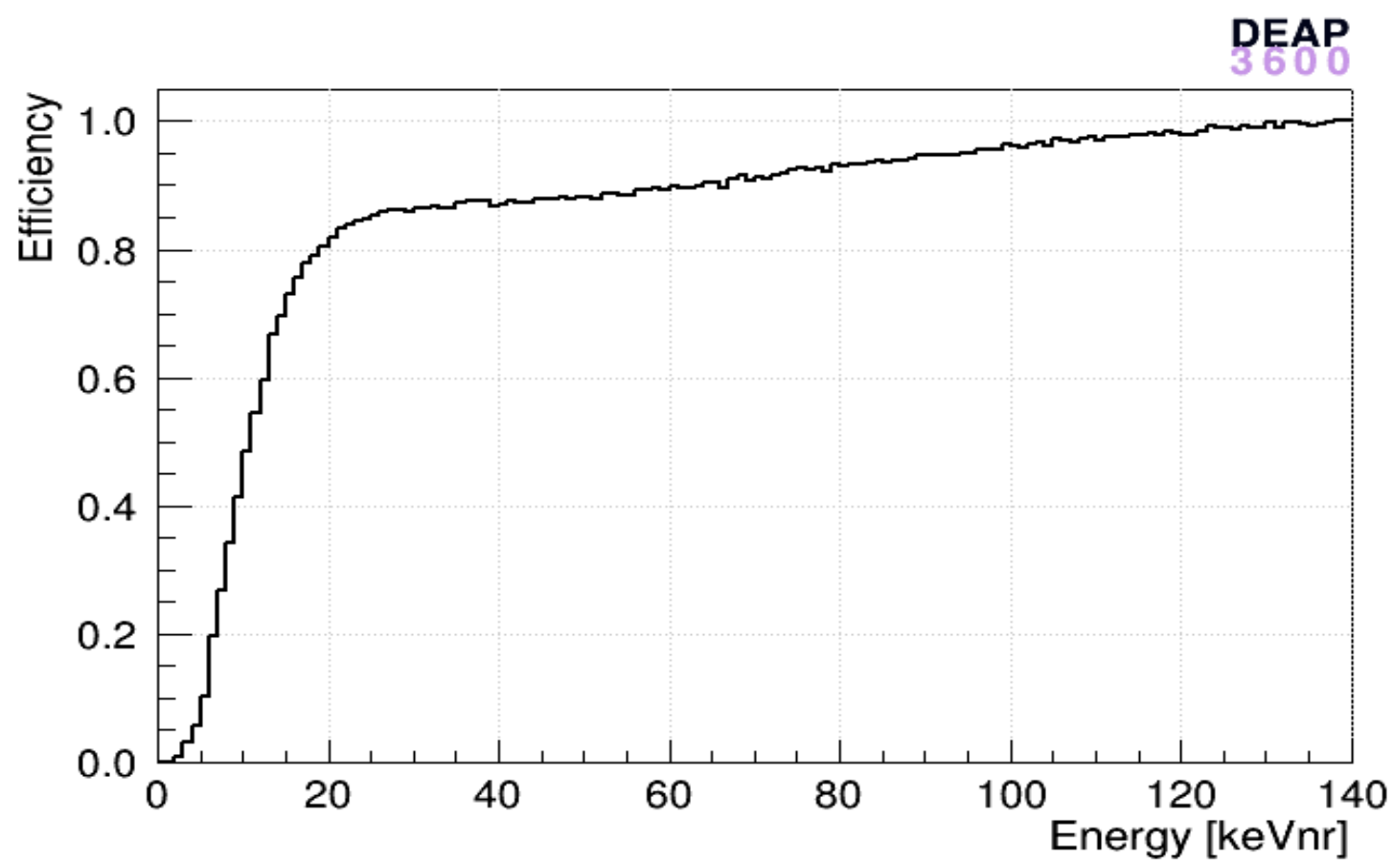

Figure 4.21: Trigger efficiency curve for low energy events with the trigger set to the low threshold settings.

\subsubsection{Exclusion Curve}

With all of the pieces discussed in this section, an exclusion curve can now be calculated. Exclusion curves are a popular way for experiments performing WIMP searches to present data as discussed in Section 1.4. Exclusion curves are used because there are only two free parameters left in the signal extraction analysis; the WIMP mass and the WIMP-nucleon cross section. Since the mass of dark matter particles are not known, the cross-section for a range of potential WIMP masses are plotted. 
The WIMP-nucleon interaction cross section can be calculated with Equation 4.18.

$$
\sigma=\frac{\sigma_{0} \cdot S_{U p}}{\text { TotalRate }}
$$

$S_{U p}$ is the $90 \%$ upper limit on the number of possible WIMPs. $S_{U p}$ can be determined by assuming the number of background events and the number of signal events in data both follow Poisson distributions; then the total number of observed events will also follow a Poisson distribution (since two Poisson distributions added together yields another Poisson distribution) given by Equation 4.19 .

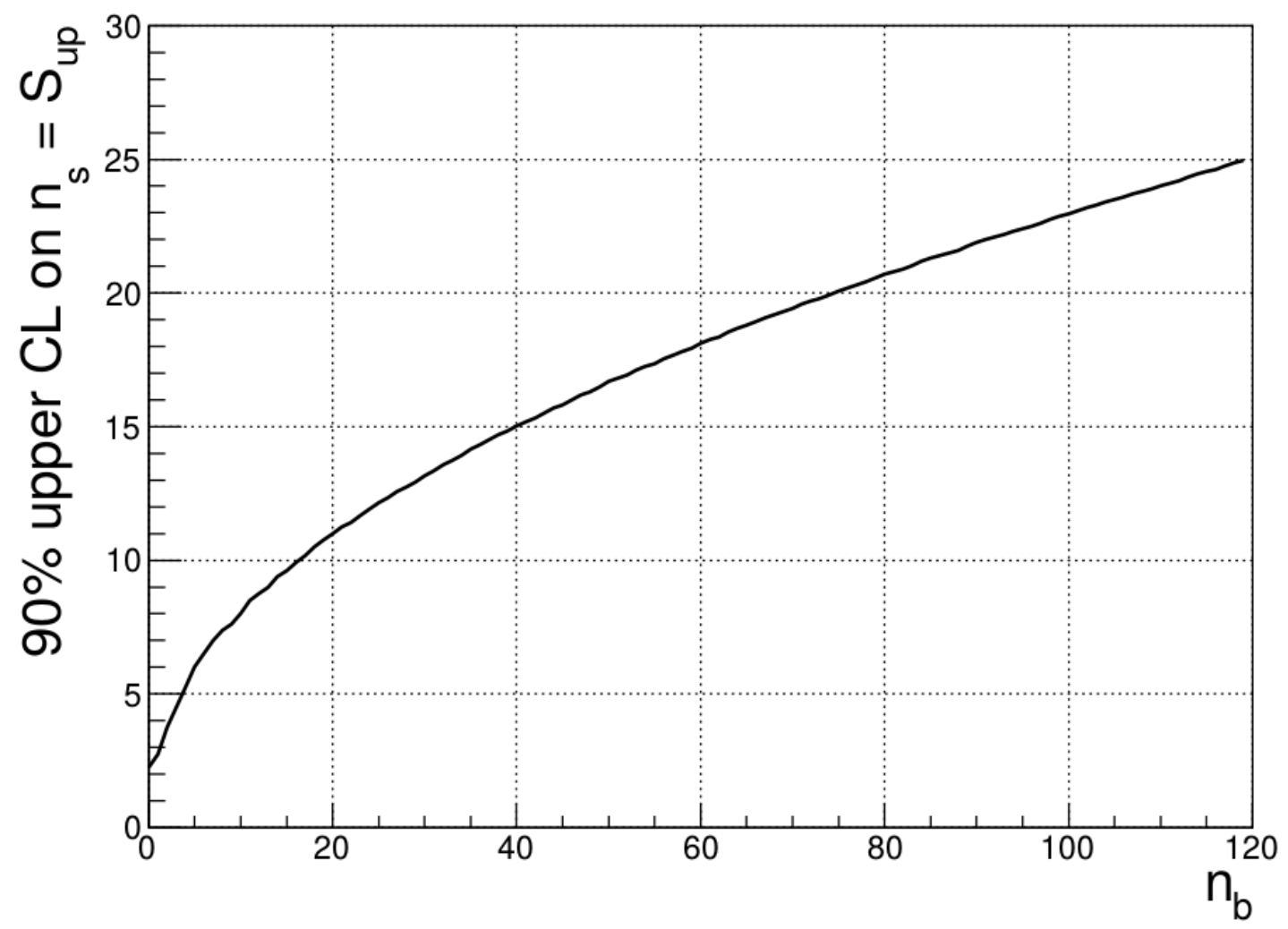

Figure 4.22: Relationship between the number of possible WIMPs observed in data given a certain number of background events observed.

$$
1-C L=e^{-S_{U p}} \cdot \frac{\sum_{m=0}^{n} \frac{\left(S_{U p}+n_{b}\right)^{m}}{m !}}{\sum_{m=0}^{n} \frac{n_{b}^{m}}{m !}} .
$$


$C L$ is the confidence level set by the researcher ( $90 \%$ for this work), $n_{b}$ is the number of background events, and $m$ is the total number of events. Equation 4.19 can then be solved numerically for $S_{U p}$ given a number of background that are left in data after background subtraction. When solved, Equation 4.19 will produce a trend seen in Figure 4.22 . 


\section{Chapter 5}

\section{Results of Low Mass WIMP Search}

\subsection{Final Exposure After Cuts}

In Section 3.2 .2 the total exposure of the low threshold data set was determined to be $9.903 \times 10^{3} \mathrm{~kg} \cdot$ days. In order to determine the final exposure, the corrected live time and fiducial mass must first be calculated first. The corrected live time accounts for the detector dead time, given by Equation 5.1 .

$$
t_{\text {DeadTime }}=N_{\text {triggers }} \times 99.838 \% \times 60 \mu \mathrm{s} .
$$

$N_{\text {triggers }}$ is the total number of triggers in the data set before cuts, the factor of $99.838 \%$ is to account for the $0.162 \%$ of triggers that are removed on average by the muon veto, and $60 \mu \mathrm{s}$ is the time between events that is set by the deltaT cut. Given that there are 1324761194 triggers in the data set, a dead time of $79356.90 \mathrm{~s}$ ( $\sim 0.918$ days $)$ is calculated. Subtracting this from the total live time of 3.02 days, the corrected live time is 2.10 days.

The fiducial mass can be calculated by multiplying the ratio ( $\epsilon$, given by Equation

5.3 of the number of events that pass all of the cuts to the number of events that pass the fiducializing cuts only by the total mass (given by Equation 5.2). A summary of 
the number of events that passed cuts in data and Monte Carlo are given in Table 5.1 .

$$
\begin{gathered}
\text { FiducialMass }=\epsilon * 3279 \mathrm{~kg} \\
\epsilon=\frac{N_{\text {PassPileUp\&Fiducial }}}{N_{\text {PassFiducial }}} .
\end{gathered}
$$

\begin{tabular}{c|c|c|c}
\hline & All Events & Events That Pass Fiducial Cuts & Events That Pass All Cuts \\
\hline \hline Data & 1324761194 & 20344367 & 13023484 \\
${ }^{39}$ Ar Monte Carlo & 1164398 & 44666 & 24050 \\
\hline
\end{tabular}

Table 5.1: Summary of events that pass the fiducializing cuts (mblikelihood cuts) and events that pass pileup+fiducializing cuts (all cuts).

Based on the values in Table 5.1 and using Equation 5.3, $\epsilon_{\text {data }}=0.640$ for data and $\epsilon_{M C}=0.538$ for Monte Carlo. In order to determine the statistical error a binomial error will be used on the Monte Carlo data. A final value of $\epsilon_{M C}=0.538 \pm 0.002$ is obtained. The systematic error can be estimated as the maximum difference between $\epsilon_{\text {data }}$ and $\epsilon_{M C}$, which is 0.1 . Combining these results yields a final value of $\epsilon_{\text {data }}=$ $0.640 \pm 0.002$ (stat.) \pm 0.1 (sys.). The fiducial mass can be calculated using Equation 5.2, which gives a fiducial mass of $(2100 \pm 330) \mathrm{kg}$.

The final exposure can be calculated by multiplying the corrected live time by the fiducial volume. The final exposure for this work is $4.41 \times 10^{3} \mathrm{~kg} \cdot$ days. $\epsilon_{\text {data }}=$ $0.640 \pm 0.002$ (stat.) \pm 0.1 (sys.) is a measure of the cut efficiency within the fiducial volume of this work.

\subsection{Background Subtraction and $\mathrm{S}_{\mathrm{Up}}$}

$S_{U p}$ can be calculated by using the background subtraction in Figure 4.18. The same background subtraction can be seen in Figure 5.1 with both the statistical error 
and total error (statistical + systematic error) shown as the green shaded area and red shaded area respectively. Using the total error value, the $90 \%$ upper limit of the number of background events can be calculated by assuming a gaussian distribution (by the central limit theorem) and creating a 90\% upper limit curve of the data presented in Figure 5.1 by scaling the $\mathrm{Y}$-axis by $y+1.28 \cdot$ TotalError. By integrating the $90 \%$ upper limit curve over the same energy range used to calculate the total rate, then multiplying the results of the integral by the live time of the data, the $90 \%$ upper limit of the number of backgrounds is obtained. The $90 \%$ upper limit of the number of background events in this data set it 590618 events.

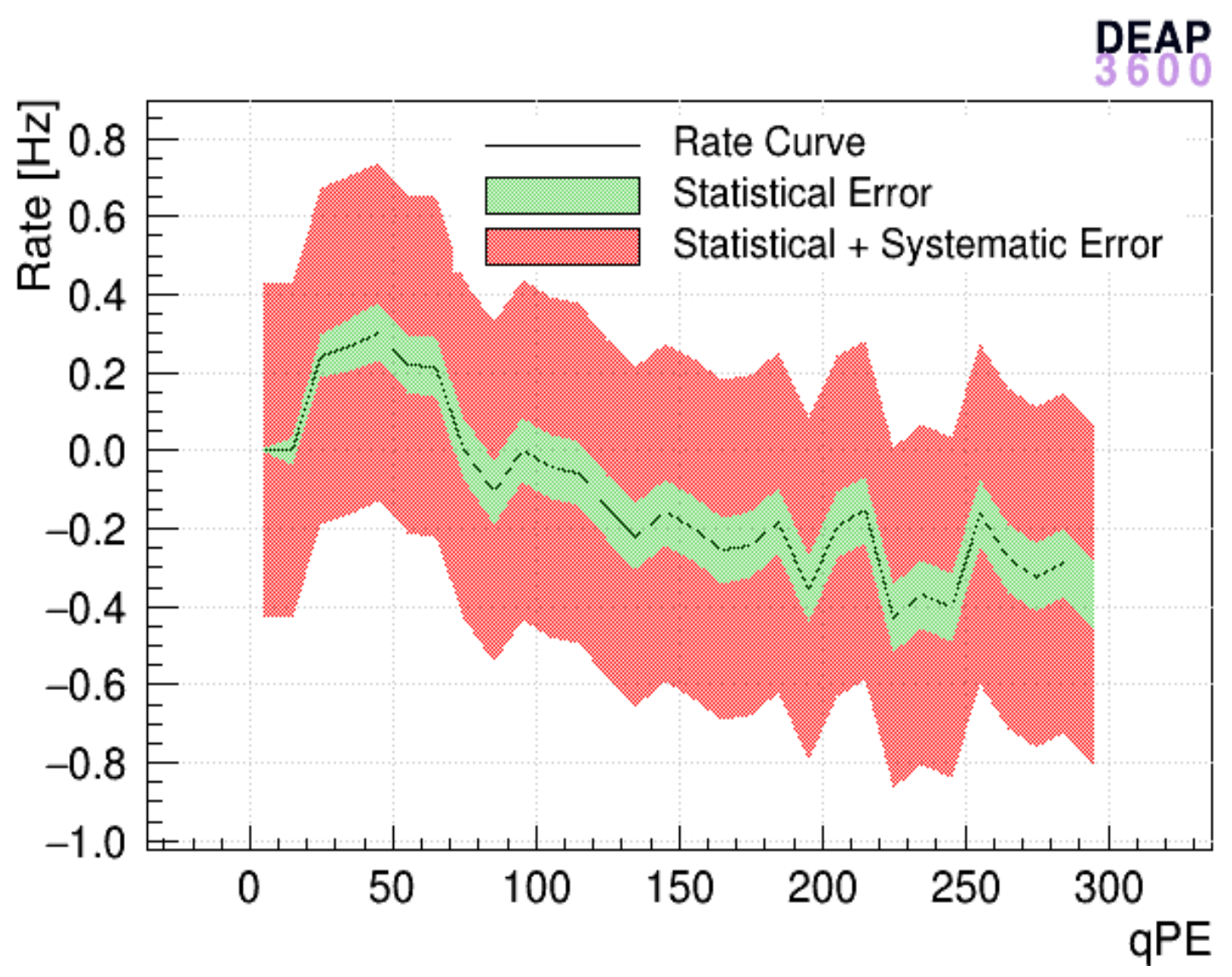

Figure 5.1: The residual rate after the total Monte Carlo contribution (discussed in Section 4.3.2 is subtracted from low threshold data. The statistical error (green) and total error (red) are shown.

If there was a WIMP signal present in the data set, it would be reflected in 
Figure 5.1 as a rate that diverges from $0 \mathrm{~Hz}$ and does not fall within the error of the background model. Since the errors on the rate curve cover a rate of $0 \mathrm{~Hz}$ across the whole energy range, it can only assumed that background events dominate this region. Because there are no signal events, the total number of observed events is equal to the number of background events. Using the calculated number of background events and observed events along with Equation 4.19, the $90 \%$ upper limit of the number of possible WIMPs can be calculated. In this data set there is a $90 \%$ upper limit of 1775 possible WIMPs.

\subsection{Low Threshold Analysis Results}

A comparison between the overall efficiency of the low threshold analysis and the standard DEAP-3600 analysis within their fiducial masses and in their WIMP search ROIs is shown in Figure 5.2. The low threshold analysis efficiency (red curve) goes to much lower energies then the standard analysis efficiency (blue curve). The main reason for this is because the standard analysis uses an $\mathrm{F}_{\text {prompt }}$ cut. Figure 2.6 shows that the trigger efficiency is dependent on $\mathrm{F}_{\text {prompt }}$, so including an $\mathrm{F}_{\text {prompt }}$ cut will introduce the corresponding trigger efficiency in the analysis. A background subtraction analysis was chosen for this work because it does not have to include a $\mathrm{F}_{\text {prompt }}$ cut, which allows for the trigger efficiency to extend to lower energies. The dominant efficiency constraint becomes the energy threshold of the trigger rather then

the $\mathrm{F}_{\text {prompt }}$ dependence of the trigger. The efficiency plateau of the low threshold data is much greater then the standard analysis efficiency. The discrepancy is mainly due to the standard analysis using both higher level and more strict cuts on their data. This results in a higher fraction of events being removed in comparison to the low threshold analysis, reducing the cut efficiency. Overall the efficiency of the low threshold data is higher then the standard analysis; but the efficiency increase comes at the cost 
of having a large number of background events left in the data when performing a background subtraction (seen in Section 5.2). The uncertainty on the low threshold efficiency is dominated by the systematic uncertainty of the cut efficiency within the fiducial mass $\left(\epsilon_{\text {data }}\right.$ in Section 5.1).

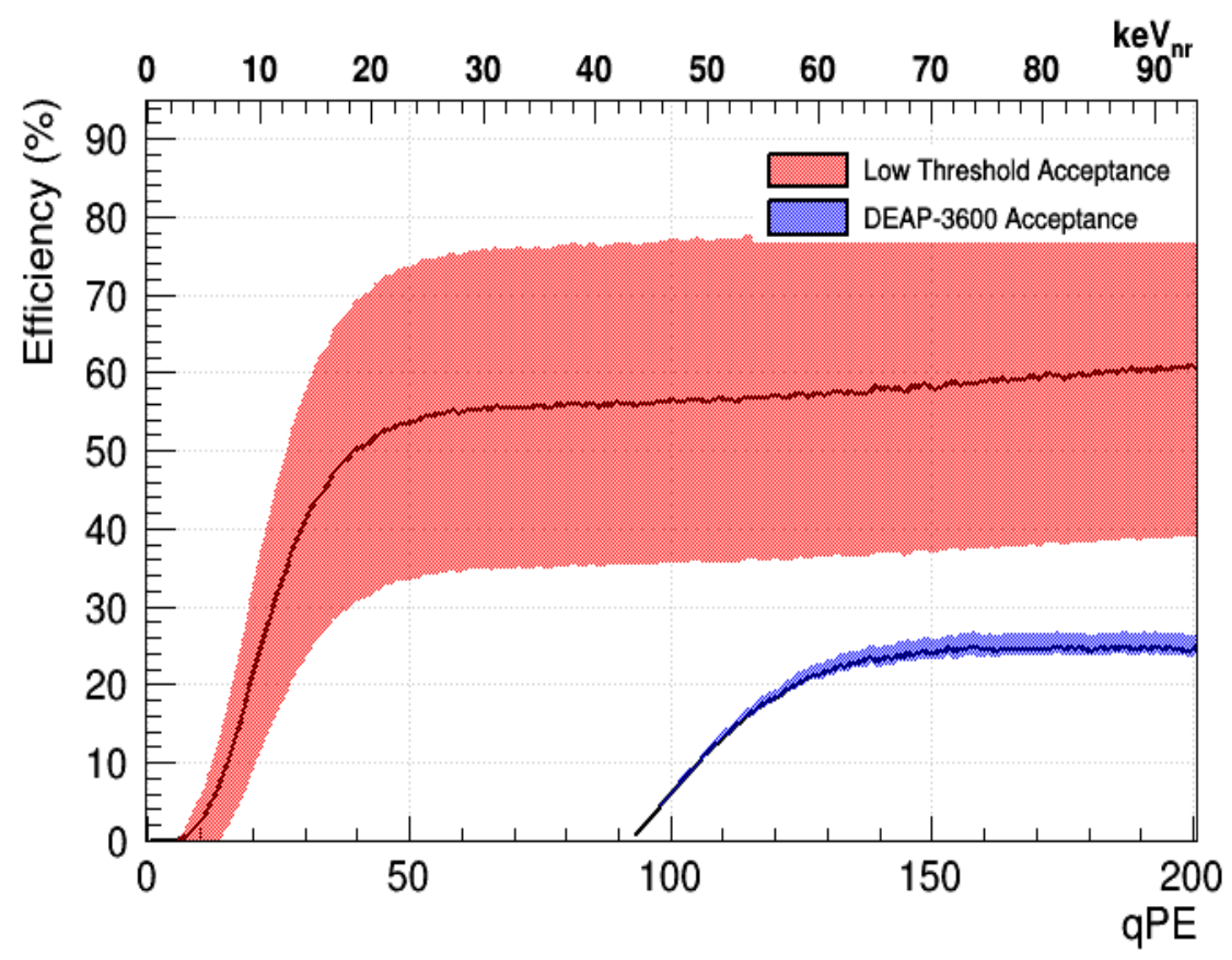

Figure 5.2: Comparison between the total efficiency of the low threshold analysis and the standard DEAP-3600 analysis [16] within their fiducial masses. The main factor that drives the efficiency of the DEAP-3600 efficiency to higher energies is the $\mathrm{F}_{\text {prompt }}$ dependence of the trigger seen in Figure 2.6.

The final results from the low threshold analysis presented in the form of and exclusion curve are given in Figure 5.3. The results of the study (black curve) are compared to the most recent results from DEAP-3600 (red curve) $[16$ and DarkSide50 (blue curve) [17.

The DEAP-3600 results are most sensitive to WIMPs with mass $100 \mathrm{GeV} / \mathrm{c}^{2}$ with a WIMP-nucleon spin independent cross section of $3.9 \times 10^{-45} \mathrm{~cm}^{2}$ at a $90 \%$ confidence 
level. In comparison, the low threshold data is most sensitive to WIMPs with mass $55 \mathrm{GeV} / \mathrm{c}^{2}$ and WIMP-nucleon spin independent cross section of $3.96 \times 10^{-42} \mathrm{~cm}^{2}$ at a $90 \%$ confidence level. This means that the standard DEAP-3600 analysis has a greater sensitivity to WIMPs with higher masses, but using the low threshold data allows for the search of WIMP with lower masses. Low mass sensitivity is reflected in Figure 5.3 as the low threshold excursion curve extend to lower WIMP masses beyond the sharp increase in WIMP-nucleon cross section of the standard analysis DEAP-3600 exclusion curve at $30 \mathrm{GeV} / \mathrm{c}^{2}$. Reasons for the discrepancies in sensitivity are due to the large number of background events that remain in the low threshold analysis. This will be discussed in the following sections.

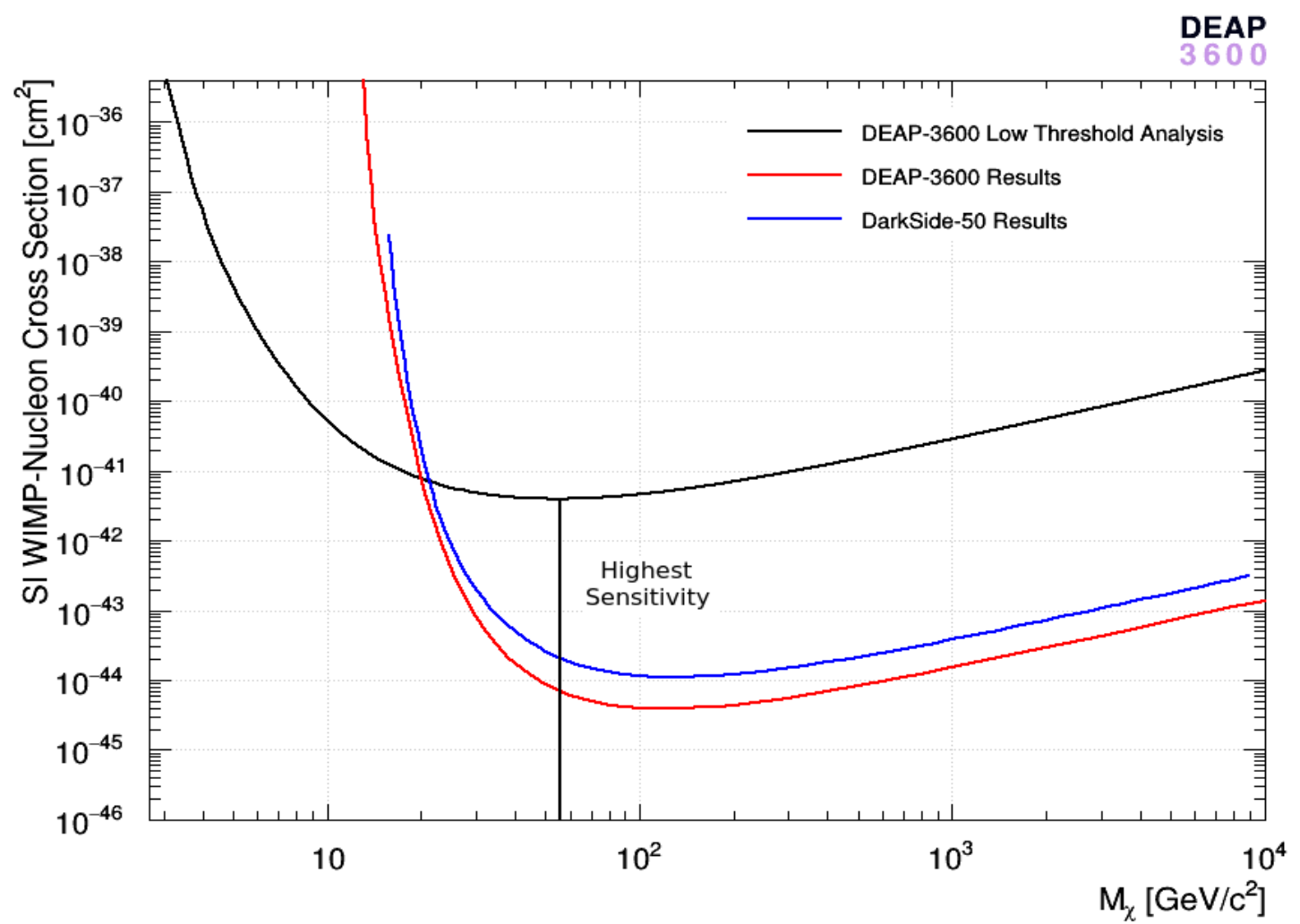

Figure 5.3: Results of low threshold analysis presented as an exclusion curve. The highest sensitivity is marked at a WIMP mass of $55 \mathrm{GeV} / \mathrm{c}^{2}$ with a WIMPnucleon spin independent cross section of $3.96 \times 10^{-42} \mathrm{~cm}^{2}$. Also plotted on here are the latest results from DEAP-3600 (with an exposure of 231 days) 16 and Darkside-50 (with an exposure of 532 days) [17. The exclusion regions at a $90 \%$ CL are represented as the area above each exclusion curve. 


\subsection{Sensitivity Study}

The purpose of the sensitivity study was to investigate how the sensitivity in DEAP-3600 changes with trigger threshold. The current DEAP-3600 trigger threshold is $10.14 \mathrm{keV}_{\mathrm{ee}}$. Comparing various threshold provides insights into how much the trigger threshold would have to modified in order to become sensitive to low mass WIMPs. The sensitivity study compares results from the low threshold analysis (blue)

with current results from DEAP-3600 (cyan) [16] and DarkSide-50 (purple) [17], as well as how the low threshold results would change if the trigger threshold was change to a range of values from $0.5 \mathrm{keV}_{\text {ee }}$ to $10 \mathrm{keV}_{\mathrm{ee}}$. A plot of the trigger thresholds (excluding DEAP-3600 and DarkSide-50) can be seen below the sensitivity study in Figure 5.4 .

The results of the sensitivity study (seen in Figure 5.4) show that the low threshold results in comparison to the DEAP-3600 results have an improved sensitivity to low mass WIMPs. However, the goal of this study is to determine which trigger threshold provides the greatest sensitivity to as low of a WIMP mass. The $0.5 \mathrm{keV}_{\text {ee }}$ trigger threshold curve (meaning that the trigger efficiency is $100 \%$ down to $0.5 \mathrm{keV}_{\mathrm{ee}}$, seen in black) provides that greatest sensitivity to low mass WIMPs. The $0.5 \mathrm{keV}_{\text {ee }}$ curve is has the lowest sensitivity to low mass WIMPs because it provides that lowest WIMPnucleon cross section value for the lowest WIMP masses. Future work would include investigating new ways to increase the threshold efficiencies in the detector down to the level of $1 \mathrm{keV}$. One approach to do this is using the random trigger data, this is discussed in Section 6.1. 

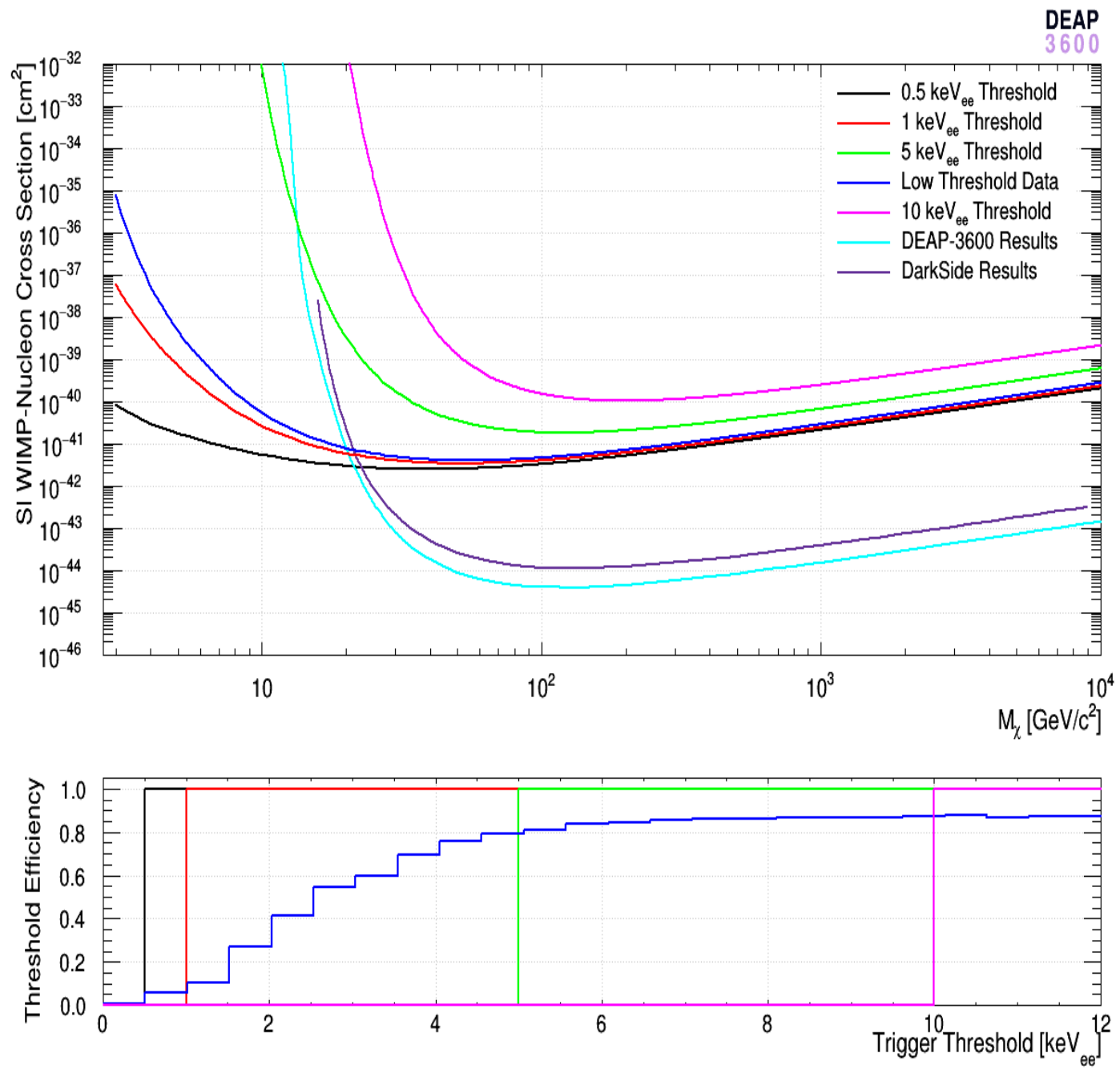

Figure 5.4: Results from the sensitivity study. The results from this data set can be seen in blue, the most recent DEAP-3600 results [16 can be seen in cyan, and the most recent DarkSide-50 results [17] can be seen in purple. The DEAP3600 and DarkSide-50 results are scaled to match the exposure of this work.

\subsection{Background Study}

The purpose of the background investigation study is to understand how the sensitivity of the low threshold data set is affected based on the number of background events observed. A future direction that is being investigated in the DEAP-3600 collaboration is whether is it worth replacing the atmospheric argon in the detector with low radioactivity underground argon for the remainder of the experiments life 
time. The specific activity of low radio activity underground argon is $6.6 \mathrm{mBq} / \mathrm{kg}[53]$, which is $\sim 144$ times lower then the current specific activity of $0.95 \mathrm{~Bq} / \mathrm{kg}$ [51]. If the atmospheric argon was replace with underground argon, the total trigger rate do to ${ }^{39} \mathrm{Ar}$ would be reduced to $22 \mathrm{~Hz}$ as well as the the pile-up rate due to ${ }^{39} \mathrm{Ar}$ ${ }^{39} \mathrm{Ar}$ coincidence trigger would reduce to $7.5 \mathrm{mHz}$. Reducing the trigger rate and the amount of pile-up will impact the deltaT spectrum seen in Figure 4.5, reducing the amount of events in close succession to one another which allows for a lower deltaT cut. If the atmospheric argon was replace with underground argon, cut efficiency would increase, since background cuts would not have to be as strict as they currently are. In turn, this would allow for more events from the data set can be kept for the final analysis.

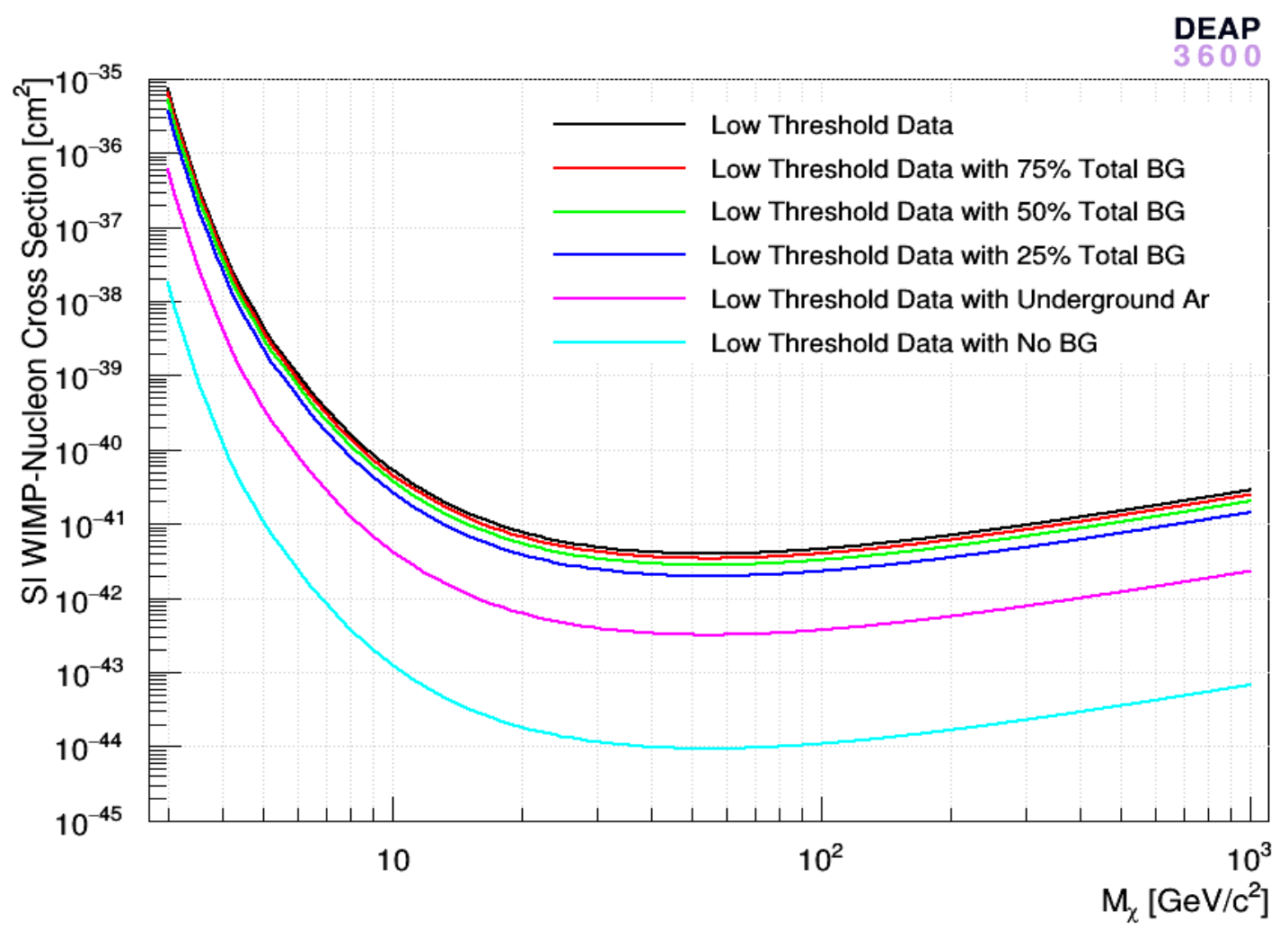

Figure 5.5: Background investigation into how the results improve with the reduction of background events. If the purified atmospheric argon in the detector was replaced with underground argon, the results would improve by approximately one order of magnitude. 
Figure 5.5 shows how the results would improve with different levels of backgrounds in the detector. If the atmospheric argon was replaced with low radioactivity underground argon the results would improve by a factor of 12.4 (the fraction difference between the black curve and the magenta curve). This would bring the maximum WIMP sensitivity down to WIMP-nucleon spin independent cross section value of $3.20 \times 10^{-43} \mathrm{~cm}^{2}$ at a $90 \%$ confidence level.

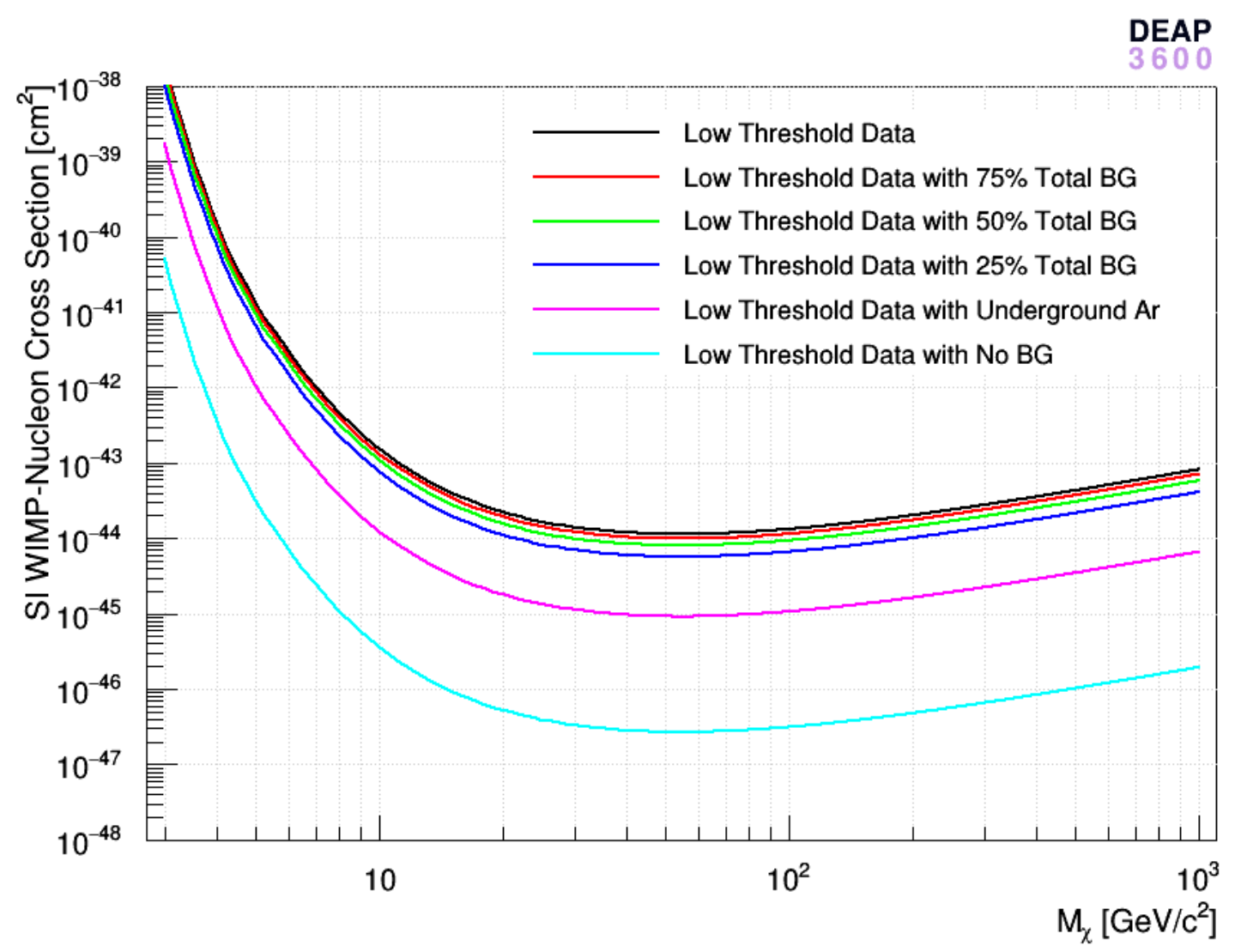

Figure 5.6: Background investigation scaled to a live time of 2 years. Based on these results, it would be beneficial to investigate switching the detector trigger to a threshold of $150 \mathrm{ADC}$ if the atmospheric argon is replaced with underground argon.

Figure 5.6 shows the same results as Figure 5.5, but now scaled to a live time of 2 years. If the atmospheric argon in DEAP-3600 was replaced with low radioactivity underground argon and the detector was run on the low threshold trigger setting for 2 years, the results would have maximum WIMP sensitivity with a WIMP-nucleon 
spin independent cross section value of $9.19 \times 10^{-46} \mathrm{~cm}^{2}$ to WIMPs with a mass of $55 \mathrm{GeV} / \mathrm{c}^{2}$. This would not only be an improvement on the WIMP-nucleon cross section sensitivity of the latest DEAP-3600 results, but would also be more sensitive to lower mass WIMPs as the exclusion curve covers a more broad range of WIMP masses. 


\section{Chapter 6}

\section{Conclusions}

In summary, the goal of this work was to perform a low energy threshold analysis using DEAP-3600 data with the goal of understanding the origin of low energy events, as well as increasing the sensitivity of the detector to low mass WIMPS. A new low threshold trigger time data processor was developed for this work to address events that do not have well calibrated trigger times. A Cherenkov radiation model was created for the PMT glass and LGs in DEAP-3600 as part of the work to help investigate low energy events. The discussion in Section 4.3 shows that the low energy events in the data set were characterized as either Cherenkov radiation, low energy retriggers or high energy retriggers. Also discussed in Section 4.3 is how to effectively remove these events from the data set using a $\mathrm{F}_{\operatorname{maxPE}}$ cut and radial cuts using mblikelihoodR. These cuts had a cut efficiency of $49.86 \%$ for removing all events that are not ${ }^{39} \mathrm{Ar}$-like (using ${ }^{39} \mathrm{Ar}$ Monte Carlo). The cut efficiency within the fiducial mass is $\epsilon_{\text {data }}=0.640 \pm 0.002$ (stat.) \pm 0.1 (sys.).

The results of the low mass WIMP search in Section 5.3 showed that lowering the hardware trigger energy threshold from 1000 ADC to 150 ADC provides an increase in sensitivity to lower mass WIMPs. The results for the low threshold data

showed that the highest sensitivity corresponds to WIMPs of mass $55 \mathrm{GeV} / \mathrm{c}^{2}$ with 
a WIMP-nucleon spin independent cross section of $3.96 \times 10^{-42} \mathrm{~cm}^{2}$ at a $90 \% \mathrm{CL}$, rather than a maximum sensitivity with a WIMP-nucleon spin independent cross section of $3.9 \times 10^{-45} \mathrm{~cm}^{2}$ at a $90 \%$ CL to WIMPs with a mass of $100 \mathrm{GeV} / \mathrm{c}^{2}$ seen in the standard physics data. This shows that the sensitivity to low mass WIMPs increases, but the lower limit on the WIMP-nucleon spin independent cross section increases due to the large amount of background events in the low energy threshold data, as discussed in Section 5.2. However, the background study in Section 5.5 shows that if the purified atmospheric argon in DEAP-3600 was replaced with low radioactivity underground argon, the lower limit of the WIMP-nucleon independent cross section would decrease to $3.20 \times 10^{-43} \mathrm{~cm}^{2}$ at a $90 \%$ CL.

In conclusion, the low energy events in the data were understood, in by creating Cherenkov model of DEAP-3600. The sensitivity in the detector was increased to include a more broad range of low mass WIMPs. A lower limit WIMP-Nucleon independent cross section of $3.96 \times 10^{-42} \mathrm{~cm}^{2}$ at a $90 \% \mathrm{CL}$ was obtained corresponding to a WIMP mass of $55 \mathrm{GeV} / \mathrm{c}^{2}$. It would be be beneficial to further investigate lowering the trigger energy threshold to 150 ADC for standard data collection if the purified atmospheric argon in DEAP-3600 was replaced with low radioactivity underground

argon. The current uncertainties of the the background subtractions dominate the analysis, which means that a $90 \%$ upper limit on the number of possible WIMP events of 1775 had to be used because a strict upper limit can not be determined. With the current atmospheric argon in the detector, the background rate due to ${ }^{39} \mathrm{Ar}$ is too high to make a trigger threshold change beneficial.

\subsection{Future Direction}

One possible way to improve the sensitivity further to low mass WIMP events would be to use the random trigger data. A small amount of work was conducted 
looking into random trigger data as part of this work. The results of the work performed are shown in Figure 6.1. In this figure, the random trigger qPE spectrum (red) is overlaid on top of the low threshold data spectrum (black). Very little differences can be seen between the random trigger data and low threshold data in this analysis. However, since there is random trigger data that is being collected at $39 \mathrm{~Hz}$ during every standard physics run, it would be beneficial to explore using this data further due to its availability. It is also beneficial due to the fact that the trigger itself is forced by the detector, meaning that there is no trigger threshold associated with it and all the information in the detector at the time of the trigger is collected. If the random trigger data can be well understood for analysis, the threshold efficiency should remain very high down to a very low energy.

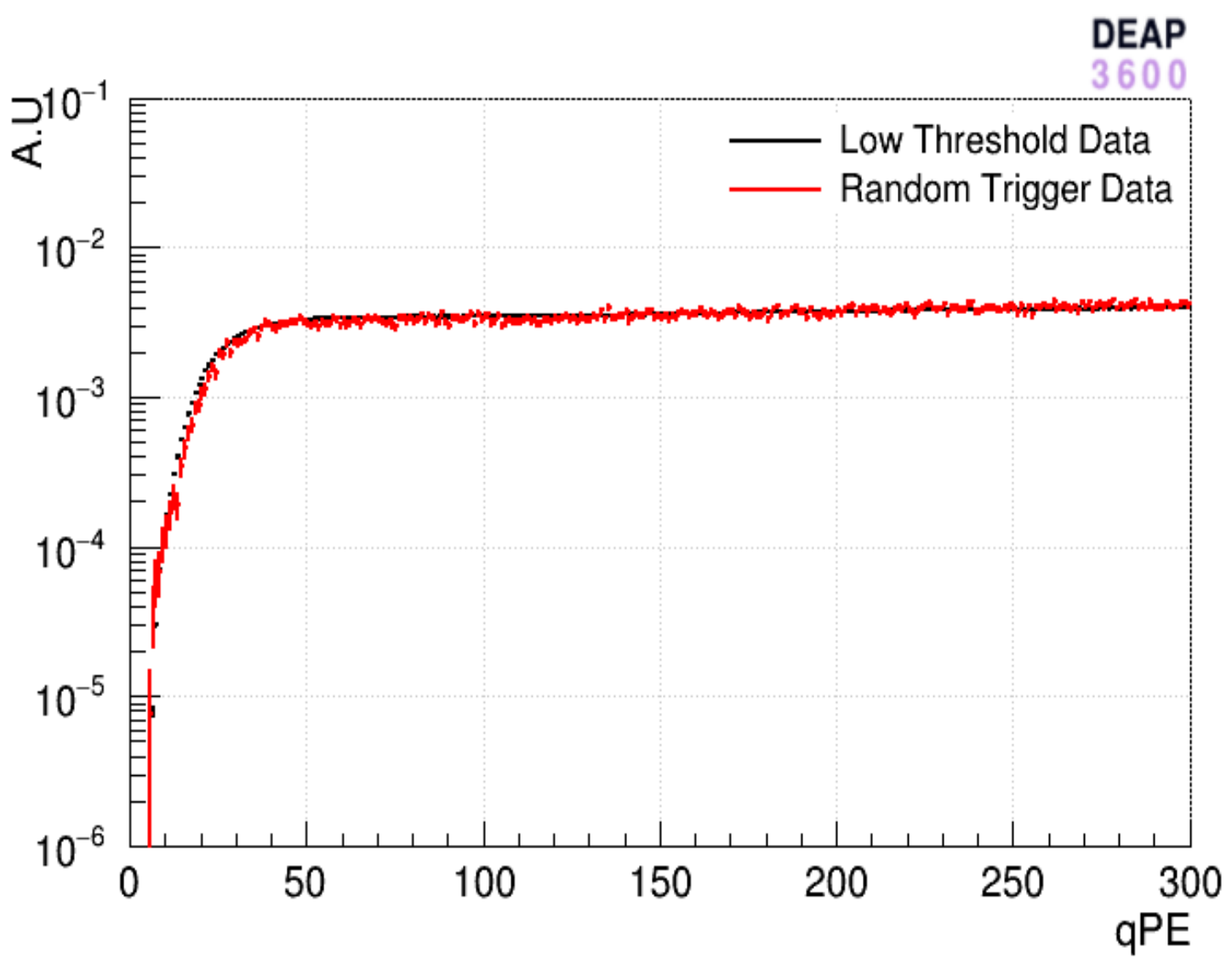

Figure 6.1: Comparison between low threshold data and random trigger data. It can be seen that they are nearly identical. 
Even though random trigger data would be very useful for a low mass WIMP search and is being continually collected along side regular physics data, there are still issues that must be solved before it can be used a low mass WIMP search analysis. The main issue being trigger shadowing that was discussed in Section 3.1. In order to be able to perform a background subtraction analysis like the one presented in this work (but with random trigger data), the live time of the run must be known. The live time is defined as the number of triggers in the data set multiplied by the time window of the trigger. If trigger shadowing is present in the data, then it is very difficult to determine the true live time of a run. Future work must to be done in order to further understand how to alleviate the trigger shadowing problem before the random trigger data can be used for a WIMP search analysis. 


\section{References}

[1] F. Zwicky, "On the Masses of Nebulae and of Clusters of Nebulae," Astrophys. J, vol. 86, p. 217, Oct. 1937.

[2] F. Zwicky, "Nebulae as gravitational lenses," Phys. Rev., vol. 51, pp. 290-290, Feb 1937.

[3] F. Zwicky, "On the large scale distribution of matter in the universe," Phys. Rev., vol. 61, pp. 489-503, Apr 1942.

[4] V. C. Rubin and J. Ford, W. Kent, "Rotation of the Andromeda Nebula from a Spectroscopic Survey of Emission Regions," Astrophys. J, vol. 159, p. 379, Feb 1970.

[5] V. C. Rubin, J. Ford, W. K., and N. Thonnard, "Rotational properties of 21 SC galaxies with a large range of luminosities and radii, from NGC $4605(\mathrm{R}=4 \mathrm{kpc})$ to UGC 2885 (R=122kpc).," Astrophys. J, vol. 238, pp. 471-487, Jun 1980.

[6] V. C. Rubin, D. Burstein, J. Ford, W. K., and N. Thonnard, "Rotation velocities of 16 SA galaxies and a comparison of Sa, SB and SC rotation properties.", Astrophys. J, vol. 289, pp. 81-104, Feb 1985.

[7] T. S. van Albada, J. N. Bahcall, K. Begeman, and R. Sancisi, "Distribution of dark matter in the spiral galaxy NGC 3198.," Astrophys. J, vol. 295, pp. 305-313, Aug 1985. 
[8] Y. Friedman and J. M. Steiner, "Gravitational deflection in relativistic newtonian dynamics," EPL (Europhysics Letters), vol. 117, p. 59001, mar 2017.

[9] D. Clowe, M. Bradač, A. H. Gonzalez, M. Markevitch, S. W. Randall, C. Jones, and D. Zaritsky, "A direct empirical proof of the existence of dark matter," The Astrophysical Journal, vol. 648, pp. L109-L113, aug 2006.

[10] Planck Collaboration, Adam, R., et al., "Planck 2015 results - i. overview of products and scientific results," $A \mathscr{E} A$, vol. 594, p. A1, 2016.

[11] Planck Collaboration, Ade, P. A. R., et al., "Planck 2015 results - xiii. cosmological parameters," A\&GA, vol. 594, p. A13, 2016.

[12] B. P. Abbott et al., "Observation of gravitational waves from a binary black hole merger," Phys. Rev. Lett., vol. 116, p. 061102, Feb 2016.

[13] S. Singirala, R. Mohanta, and S. Patra, "Singlet scalar dark matter in u(1)b-l models without right-handed neutrinos," The European Physical Journal Plus, vol. 133, p. 477, Nov 2018.

[14] G. Bertone and T. M. P. Tait, "A new era in the search for dark matter," Nature, vol. 562, no. 7725 , pp. 51-56, 2018.

[15] T. Bringmann and C. Weniger, "Gamma ray signals from dark matter: Concepts, status and prospects," Physics of the Dark Universe, vol. 1, no. 1, pp. $194-217$, 2012. Next Decade in Dark Matter and Dark Energy.

[16] R. Ajaj et al., "Search for dark matter with a 231-day exposure of liquid argon using deap-3600 at snolab," Submitted to Phys. Rev. D, in press.

[17] P. Agnes et al., "Darkside-50 532-day dark matter search with low-radioactivity argon," Phys. Rev. D, vol. 98, p. 102006, Nov 2018. 
[18] C. Amole et al., "Dark matter search results from the complete exposure of the pico-60 c3f8 bubble chamber," in press.

[19] G. Adhikari et al., "Search for a dark matter-induced annual modulation signal in nai(tl) with the cosine-100 experiment," in press.

[20] R. Bernabei et al., "First results from dama/libra and the combined results withdama/nai," The European Physical Journal C, vol. 56, pp. 333-355, Aug 2008.

[21] E. Aprile et al., "Dark matter search results from a one ton-year exposure of xenon1t," Phys. Rev. Lett., vol. 121, p. 111302, Sep 2018.

[22] R. Agnese et al., "Results from the super cryogenic dark matter search experiment at soudan," Phys. Rev. Lett., vol. 120, p. 061802, Feb 2018.

[23] D. S. Akerib et al., "Results from a search for dark matter in the complete lux exposure," Phys. Rev. Lett., vol. 118, p. 021303, Jan 2017.

[24] X. Cui et al., "Dark matter results from 54-ton-day exposure of pandax-ii experiment," Phys. Rev. Lett., vol. 119, p. 181302, Oct 2017.

[25] C. Patrignani et al., "Review of Particle Physics," Chin. Phys., vol. C40, no. 10, p. 100001, 2016.

[26] P. Bull et al., "Fundamental physics with the square kilometer array," Submitted to $P A S A$, in press.

[27] S. J. Asztalos et al., "Squid-based microwave cavity search for dark-matter axions," Phys. Rev. Lett., vol. 104, p. 041301, Jan 2010.

[28] J. L. Feng, A. Rajaraman, and F. Takayama, "Superweakly interacting massive particle dark matter signals from the early universe," Phys. Rev. D, vol. 68, p. 063504, Sep 2003. 
[29] P. Cushman et al., "Snowmass cf1 summary: Wimp dark matter direct detection," in press.

[30] K. Freese, M. Lisanti, and C. Savage, "Colloquium: Annual modulation of dark matter," Rev. Mod. Phys., vol. 85, pp. 1561-1581, Nov 2013.

[31] P. Agnes et al., "Low-mass dark matter search with the darkside-50 experiment," Phys. Rev. Lett., vol. 121, p. 081307, Aug 2018.

[32] P. Agnes et al., "First results from the darkside-50 dark matter experiment at laboratori nazionali del gran sasso," Physics Letters B, vol. 743, pp. 456 - 466, 2015 .

[33] D. McKinsey, "The mini-clean experiment," Nuclear Physics B - Proceedings Supplements, vol. 173, pp. 152 - 155, 2007. Proceedings of the 7th UCLA Symposium on Sources and Detection of Dark Matter and Dark Energy in the Universe.

[34] New Experiments with Spheres-Gas, "News-g at snolab," 2019. [Online; accessed July 8, 2019].

[35] SNOLAB User's Handbook. rev. 2, Published Online, 2006, http://snolab2008.snolab.ca/snolab_users_handbook_rev02.pdf.

[36] Hamamatsu Photonics K.K, Hamamatsu R5912 photomultiplier tube datasheet, 1998.

[37] P.-A. Amaudruz et al., "Design and construction of the deap-3600 dark matter detector," Astroparticle Physics, vol. 108, pp. 1 - 23, 2019.

[38] D.-M. Mei, Z.-B. Yin, L. Stonehill, and A. Hime, "A model of nuclear recoil scintillation efficiency in noble liquids," Astroparticle Physics, vol. 30, no. 1, pp. $12-17,2008$. 
[39] T. Heindl et al., "The scintillation of liquid argon," EPL (Europhysics Letters), vol. 91, p. 62002, Sep 2010.

[40] T. Doke et al., "Absolute scintillation yields in liquid argon and xenon for various particles," Japanese Journal of Applied Physics, vol. 41, pp. 1538-1545, Mar 2002

[41] A. Hitachi et al., "Effect of ionization density on the time dependence of luminescence from liquid argon and xenon," Phys. Rev. B, vol. 27, pp. 5279-5285, May 1983.

[42] A. Hitachi, T. Doke, and A. Mozumder, "Luminescence quenching in liquid argon under charged-particle impact: Relative scintillation yield at different linear energy transfers," Phys. Rev. B, vol. 46, pp. 11463-11470, Nov 1992.

[43] H. Cao et al., "Measurement of scintillation and ionization yield and scintillation pulse shape from nuclear recoils in liquid argon," Phys. Rev. D, vol. 91, p. 092007 , May 2015.

[44] R. W. Stoenner, O. A. Schaeffer, and S. Katcoff, "Half-Lives of Argon-37, Argon39, and Argon-42," Science, vol. 148, pp. 1325-1328, Jun 1965.

[45] M. Wang et al., "The AME2016 atomic mass evaluation (II). tables, graphs and references," Chinese Physics C, vol. 41, mar 2017.

[46] J. Calvo et al., "Backgrounds and pulse shape discrimination in the ArDM liquid argon TPC," Journal of Cosmology and Astroparticle Physics, vol. 2018, pp. 011011, dec 2018.

[47] Nucleonica, "Reduced decay scheme, 19 k 40," 2019. [Online; accessed July 2, 2019]. 
[48] Nuclear Forensic Search Project, "Decay chain of isotope th-232," 2019. [Online; accessed July 2, 2019].

[49] Nuclear Forensic Search Project, "Decay chain of isotope u-238," 2019. [Online; accessed July 2, 2019].

[50] S. Chu et al., "Lund/lbnl nuclear data search," 1999. [Online; accessed July 9, 2019].

[51] R. Ajaj et al., "Electromagnetic backgrounds and potassium-42 activity in the deap-3600 dark matter detector," Submitted to Phys. Rev. D, in press.

[52] J. Lewin and P. Smith, "Review of mathematics, numerical factors, and corrections for dark matter experiments based on elastic nuclear recoil," Astroparticle Physics, vol. 6, no. 1, pp. $87-112,1996$.

[53] J. Xu, F. Calaprice, C. Galbiati, A. Goretti, G. Guray, T. Hohman, D. Holtz, A. Ianni, M. Laubenstein, B. Loer, C. Love, C. Martoff, D. Montanari, S. Mukhopadhyay, A. Nelson, S. Rountree, R. Vogelaar, and A. Wright, "A study of the trace 39ar content in argon from deep underground sources," Astroparticle Physics, vol. 66, pp. $53-60,2015$.

[54] NIST Chemistry WebBook, "Isothermal properties for argon," 2019. [Online; accessed July 3, 2019]. 


\section{Appendix A}

\section{Determining the Mass of Liquid}

\section{Argon}

The mass of LAr in the detector can be determined by using the relationship between mass and volume through density. Since the AV is not completely full of LAr the actual volume will be the volume of the spherical AV minus the the cap of the sphere that does not contain any LAr. This can be calculated using Equation A.1.

$$
V_{L A r}=\frac{4}{3} \pi R^{3}-\frac{\pi h^{2}}{3}(3 R-h)
$$

Given that the AV has a cold radius of $\mathrm{R}=(845.6 \pm 1.9) \mathrm{mm}$ and the LAr fill level is $\mathrm{Z}=(551 \pm 50) \mathrm{mm}$, the height of the cap, h, can be determined.

$$
\begin{aligned}
h & =R-Z \\
& =845.6 \mathrm{~mm}-551 \mathrm{~mm} \\
& =294.6 \mathrm{~mm} .
\end{aligned}
$$


Plugging this into Equation A.1, a volume of $(2.329 \pm 0.067) \mathrm{m}^{3}$ is obtained. The density of the LAr in the AV is $(1408 \pm 9) \mathrm{kg} \mathrm{m}^{-3}$ [54]. Using the values for the volume and density of LAr, the mass of LAr in DEAP-3600 before fiducialization is determined to be $(3279 \pm 97) \mathrm{kg}$. 